\title{
Anti-trafficking programs in South Asia: Appropriate activities, indicators and evaluation methodologies [Arabic]
}

Dale Huntington

Follow this and additional works at: https://knowledgecommons.popcouncil.org/departments_sbsr-rh How does access to this work benefit you? Let us know!

\section{Recommended Citation}

Huntington, Dale. 2002. "Anti-trafficking programs in South Asia: Appropriate activities, indicators and evaluation methodologies [Arabic]," Summary report of a technical consultative meeting. New Delhi: Population Council. 


\section{بزامج مكافَحة الاتجار بالبشز \\ في منطقة جنوب آسيا South Asia Region}

الأشطة المناسبة،

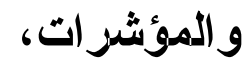
ومنهجيات التقييم

$$
\text { تلقزيز موجز حول الاجتئني }
$$




\section{بز بزامج مكافحة الاتجار بالبشز في منطقة جنوب آسيا South Asia Region}

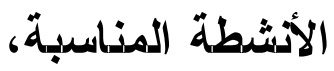

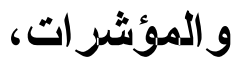

ومنهجيات التقييزم

تقزيز موجز حول

الاجتماع الفني التثاوري

1

كتاماتدو، نيبال Kathmandu, Nepal

إعداد ديل هاتتجتون Dale Huntington

أعضاء اللجنة التنسيقية

ناتديتا باروا، صندوق الأمم المتحدة لإدماء المزأة Nandita Baruah, UNIFEMسيلين كوستيللي دالي

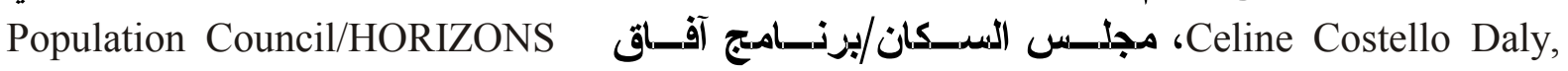

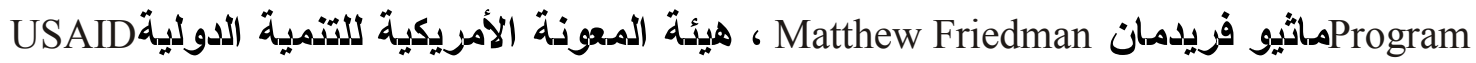

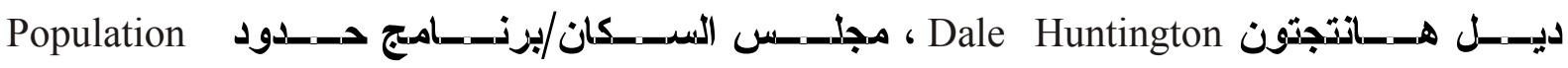

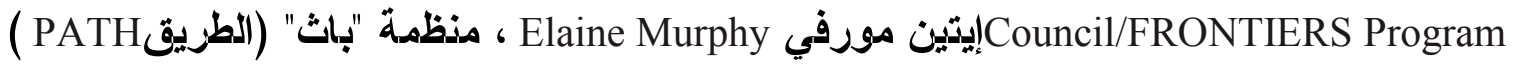

مجلس السكان Population Council:

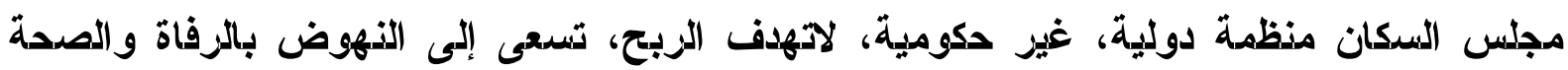

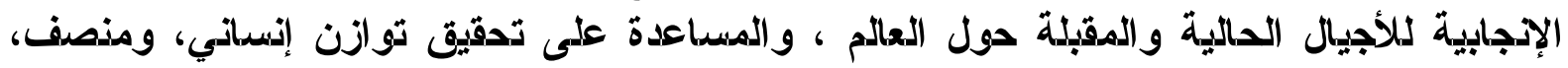

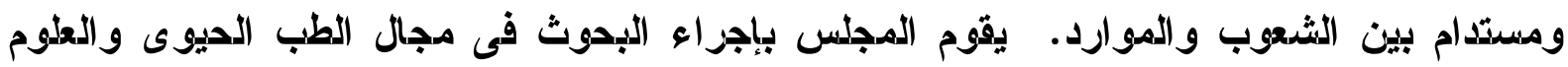

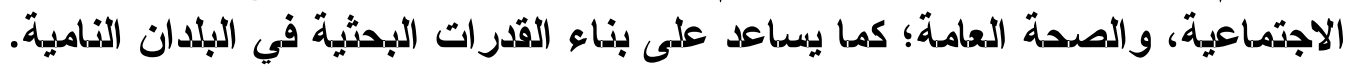

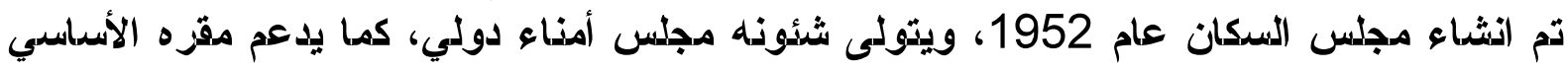
الموجود بمدينة نيويورك شبكة من المكاتب الإقليمية و القطرية حول العائ العالم. 
يقترح عذد الإثدارة إلى التقريز كتابة ما يلي:

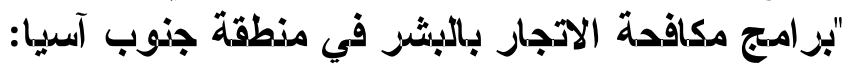

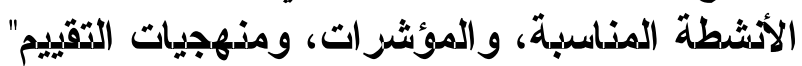

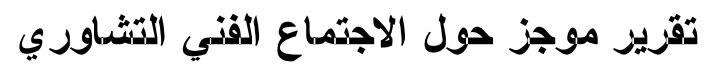

هuntington, Dale هاتتجتون، ديل

Population Council, New Delhi مجلس السكان، نيودلهي 2002 (March مارس)

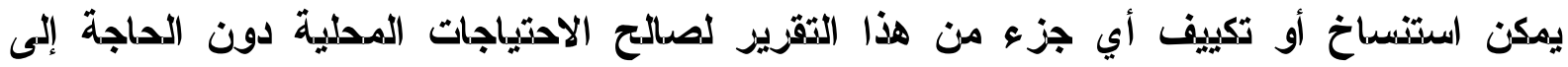

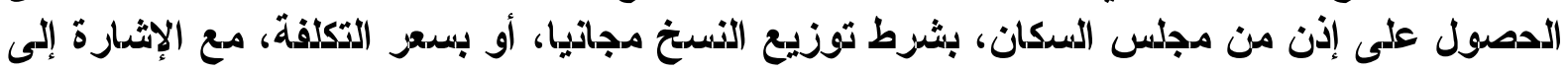

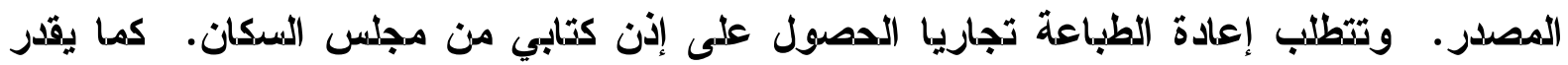

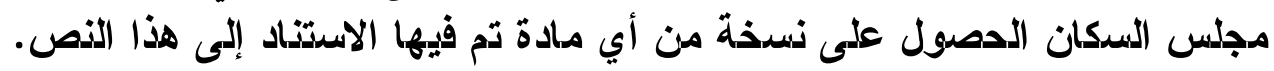

Office of تم إعداد هذا الإصدار بدعم من مكتب السكان بهيئة المعونة الأمزيكية للتنميـة الدوليـة Population of the United States Agency for International Development (USAID) Frontiers in Reproductive بمقتضى اتفاقية التعاون مع برنامج حدود حول الصدة الإنجابية

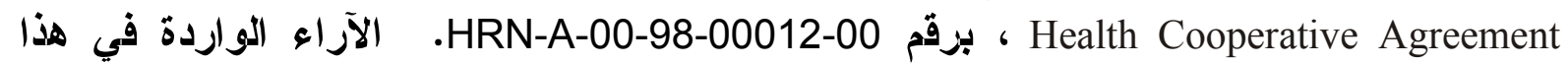
الإصدار تعبز عن المؤلفين، ولا تعكس بالضرورة وجهة نظر هيئة المعونة الأمزيكية للتنمية

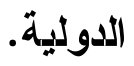

2002، جميع الدقوق مدفوظة لمجلس السكان USAID 
.5

6

شكلر وتقديز

8

توضيح المفاهيم و المصطلحات

8

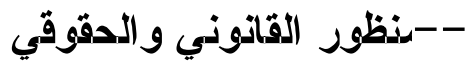
التصركات و المخاطز

24 تحديد ذماذج للتذخل

24.

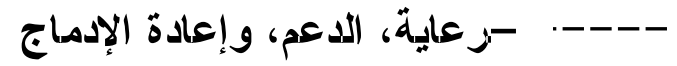




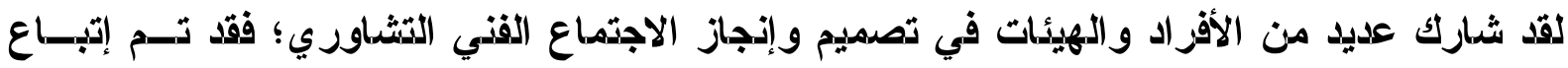

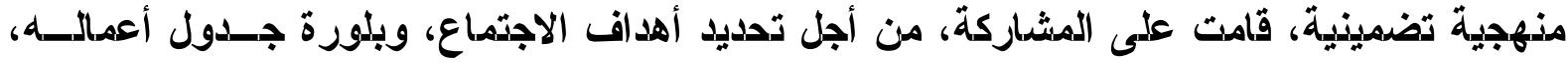

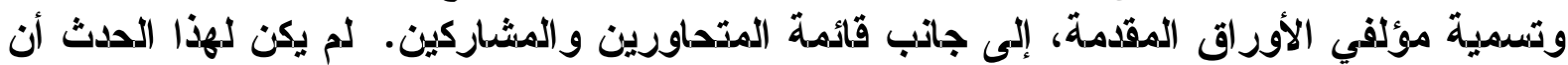

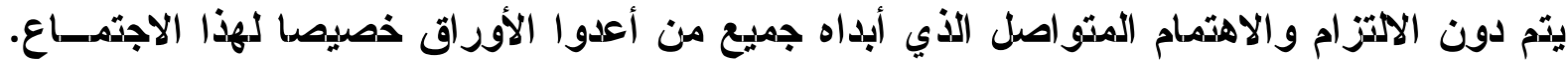

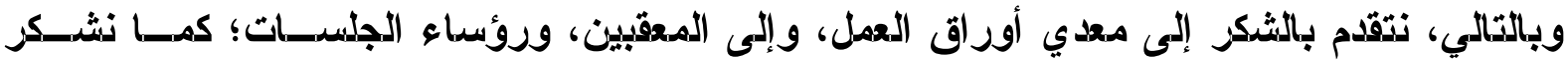

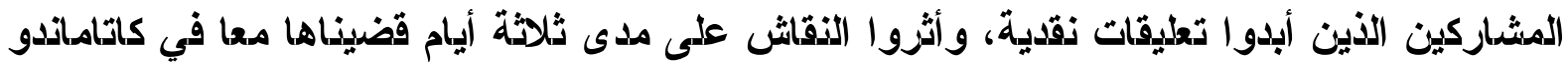
Kathmandu, Nepal. بنيبال الفين

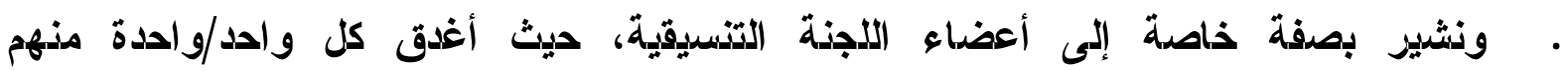

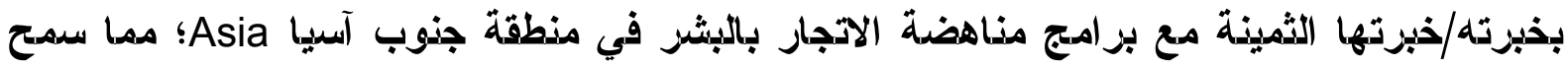
بمشاركة واسعة الذطاق في هذا الاجتماع.

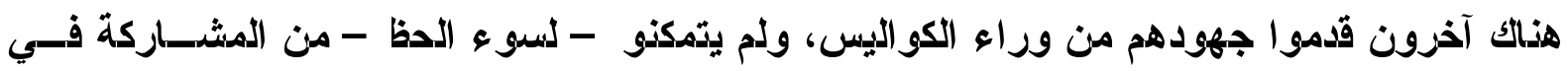

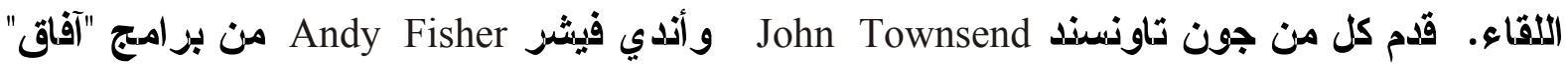

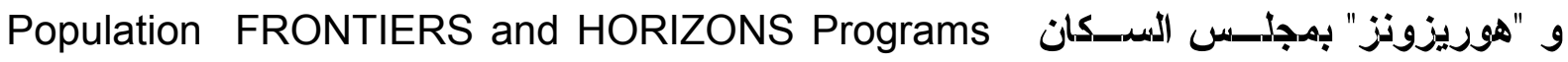
هاركو جأهil's

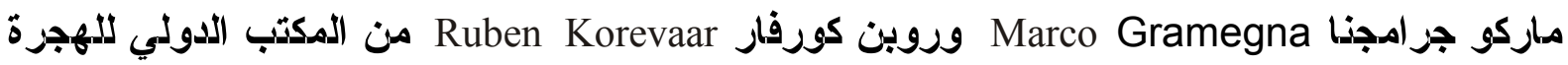

IOM offices في كل من جينيف Geneva وداكا Dhaka في التخطيط الاستراتيجي الخاص بتحديد أهداف اللقاء.

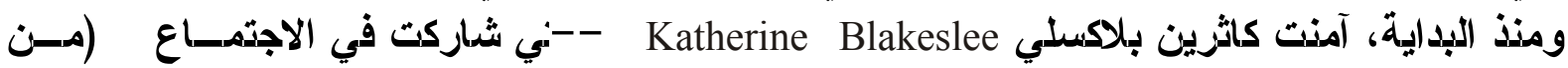

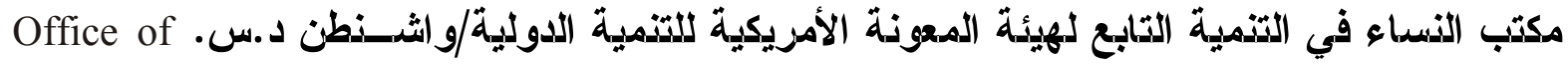
Carla Barbiero (Women in Development, USAID/Washington, D.C.

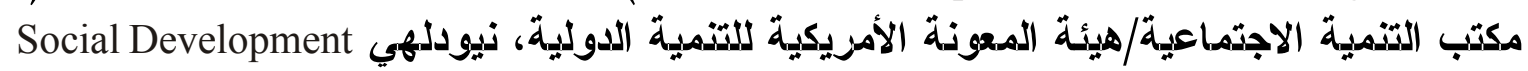
هن ( Office, USAID/New Delhi من أجل تدقيقه.

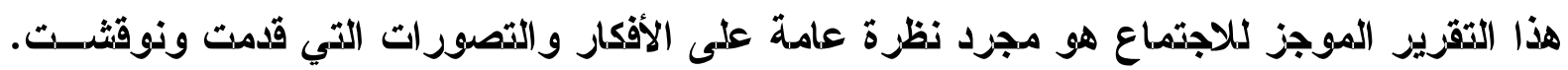

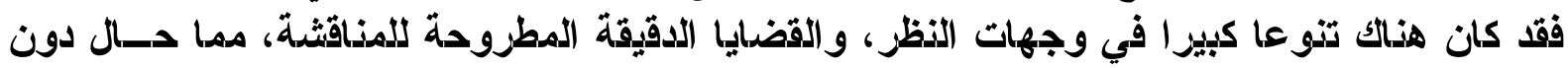

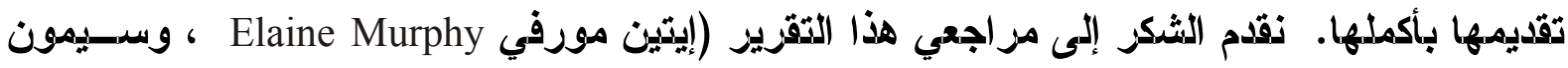

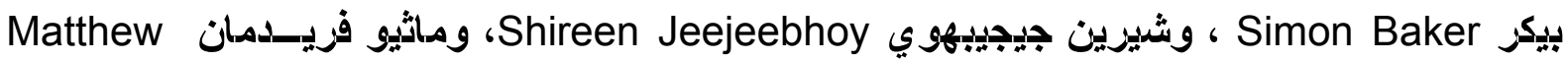

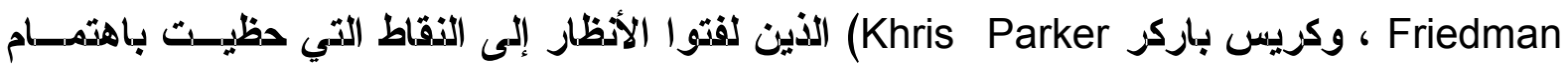

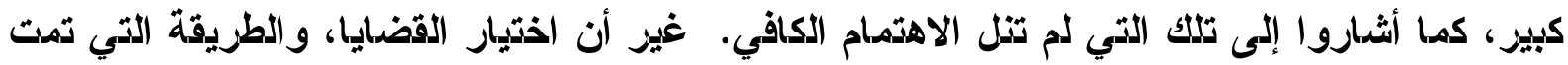

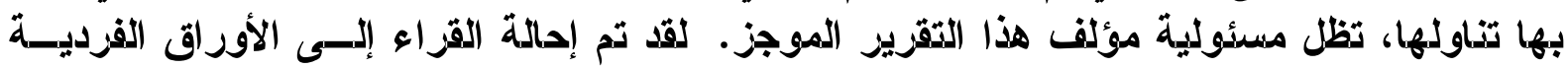

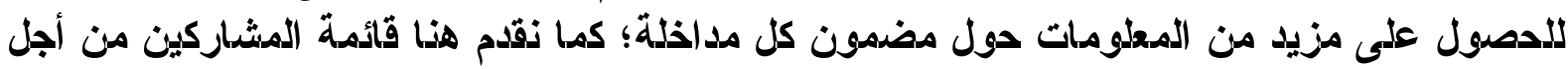
الاستفادة بها في المناقثشات التي ستثلعلق بالمتابعة. 


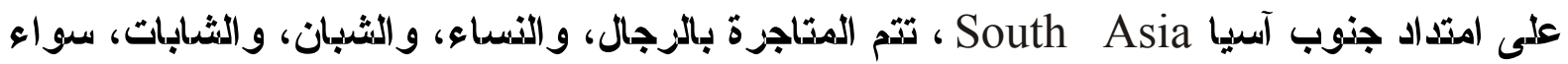

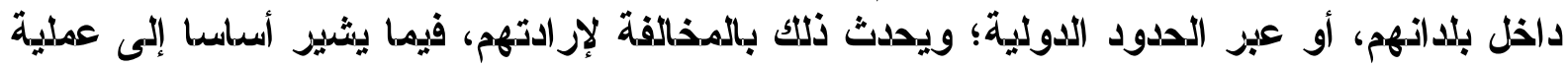

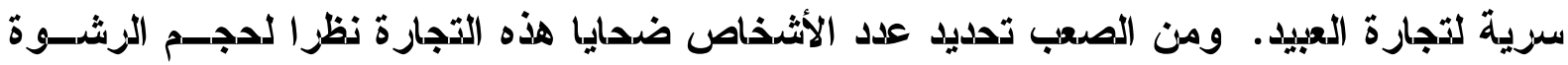

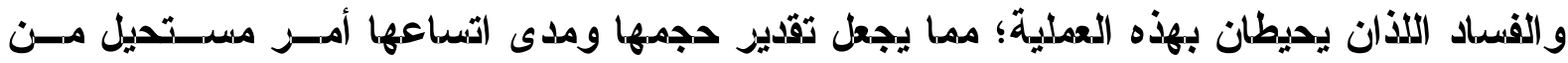

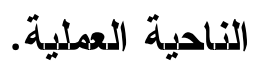

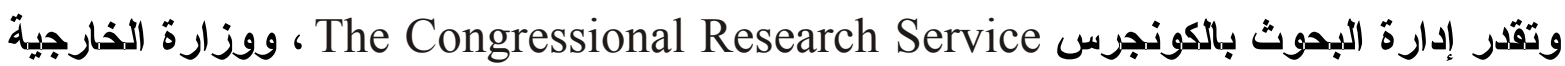

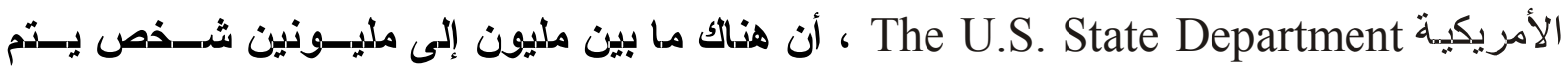

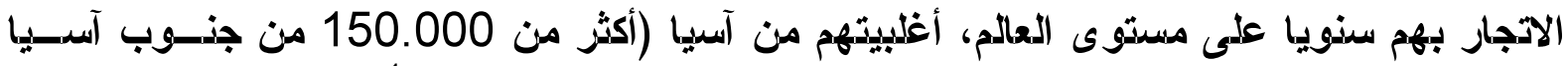
South Asia

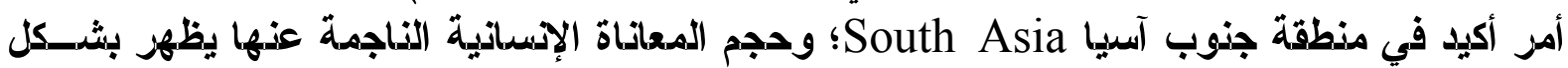

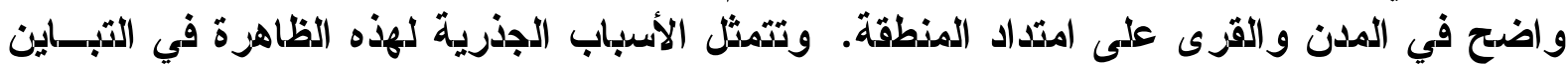

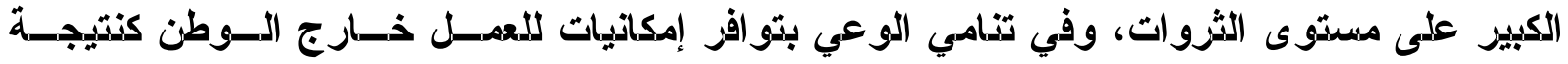

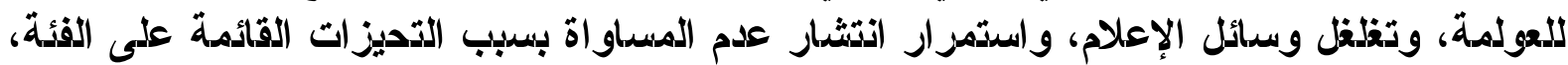

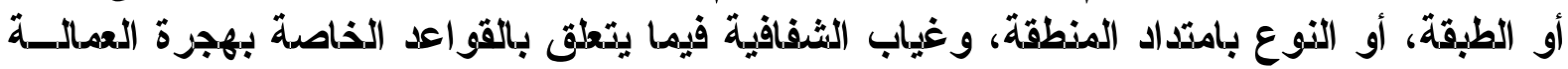

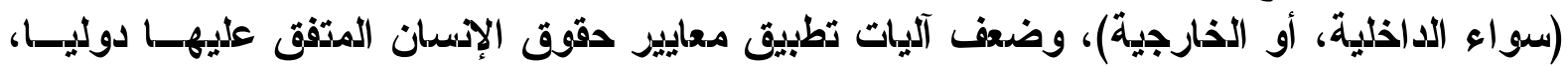
و المكاسب الباهظة التي يدصل عليها تجار البشر.

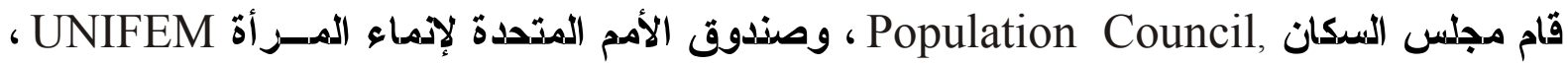

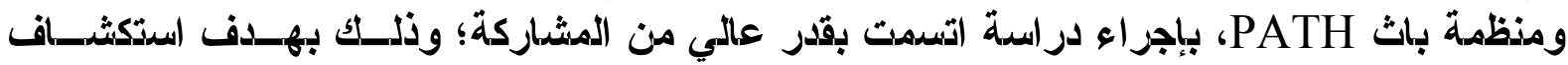

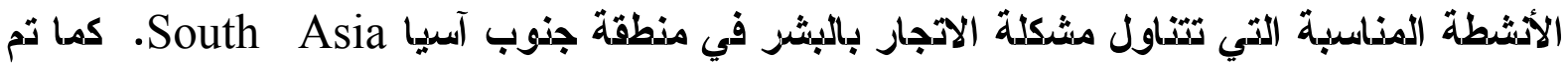

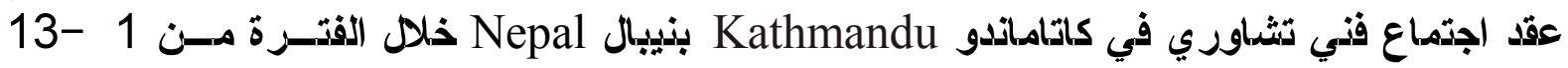

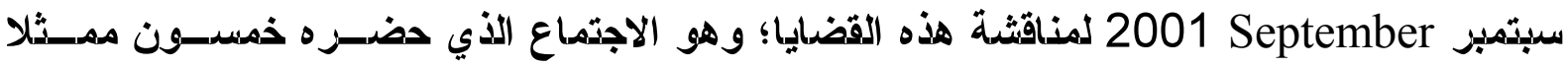

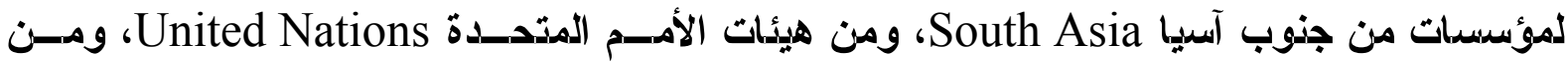
المنظمات غيز الدكومية المحلية و الدولية NGOs.

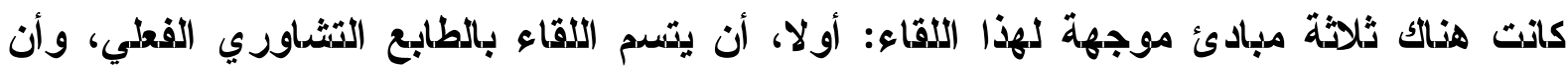

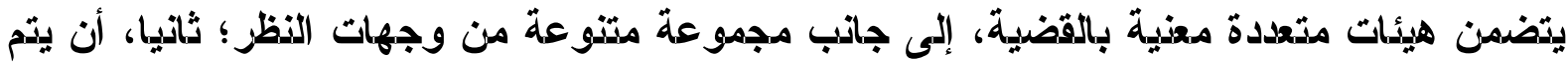

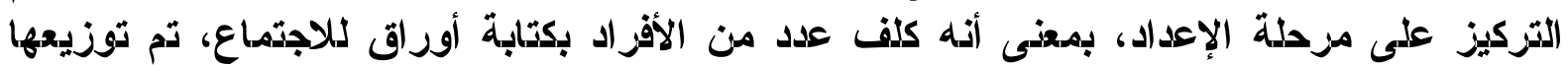

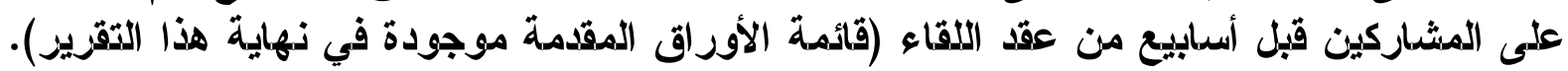

1 Congressional Research Service, Report 98-649 “Trafficking in Women and Children: The US and International Response" May 10, 2000; www.usinfo.state.gov/topical/global/traffic خدمة بدوث الكونجزس، التقزير رقم 8 -649 "الاتجار بالنساء والأطفال: رد الفعل الأمريكى والدولى" 10 مايو 
أما المبدأ الموجه الثالث، فقد تمثل في الالتزام بتطويز وتصميم نموذج لتقييزم عمليات التدخل

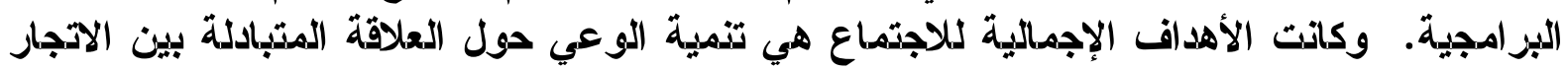

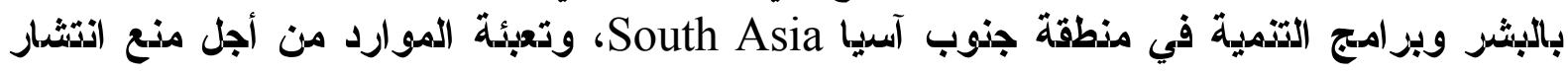

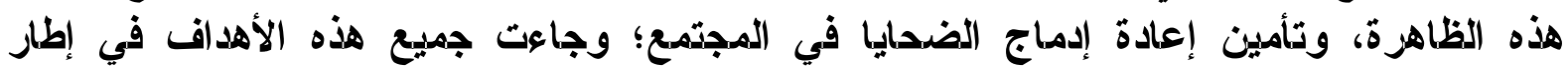

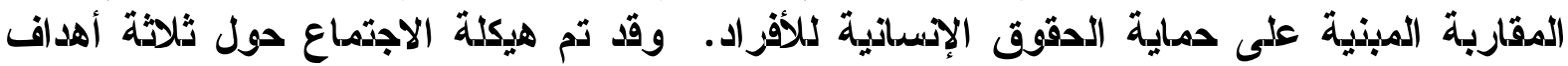
عملية: 1. توضيح الأطر المفاهيمية المستعملة في تعريف الاتجار بالبشر.

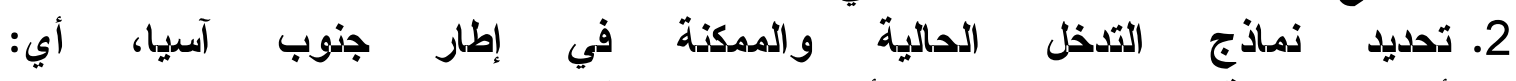

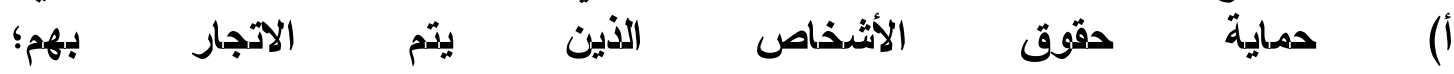

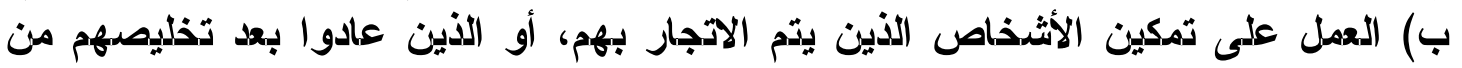

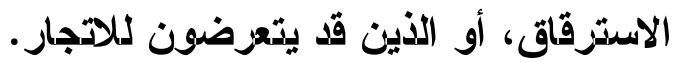

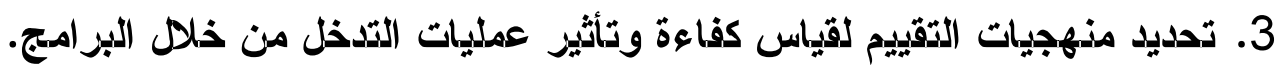

يقوم التقريز الدالي بتلذيص الذقاط الأساسية التي تضمنتها كل ورقة من الأوراق المقدمة،

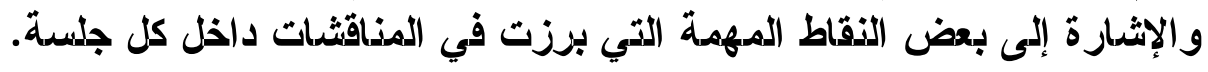




\section{- المنظور القانوني والدقوقي القانيون الدولي والاتفاقيات المتعلقة بالاتجار بالبثن}

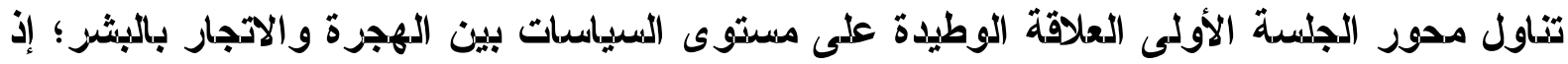

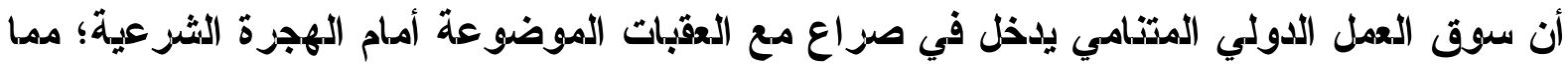

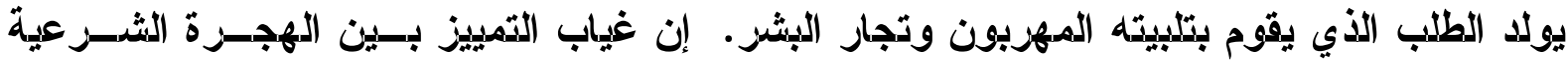

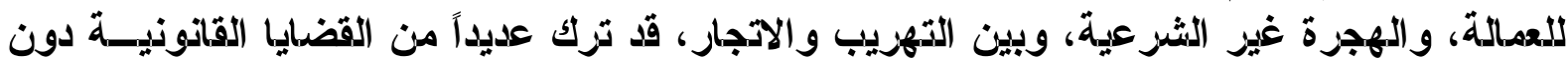

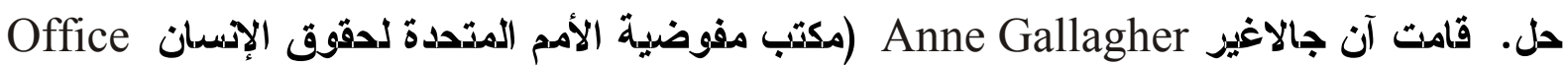

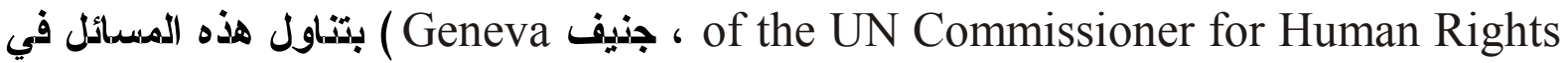
ورقتها التي تضمنت نظرة تاريخية عامة حول القانون الدولي للقرن العشـرين الذهاص بالاتجسار بالبشز من وجهة نظر حقوقية.

يعد الدظز الدولي للاسترقاق والاتجار بالعبيد من أولى الدقوق التي أقز بها القانون الدولي. فئناك

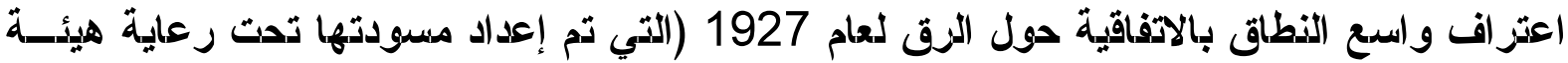

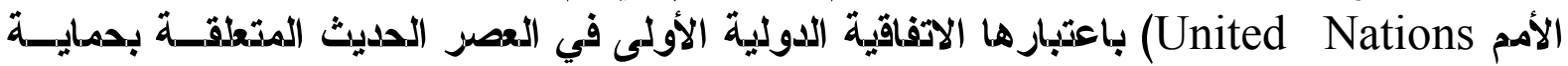

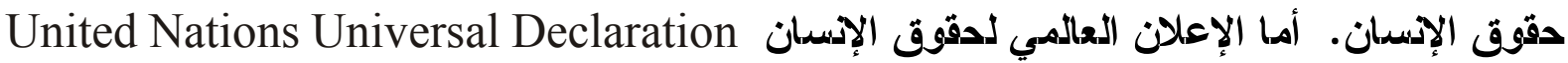

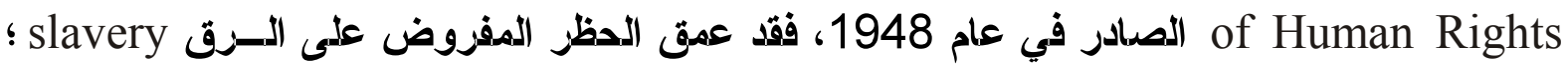

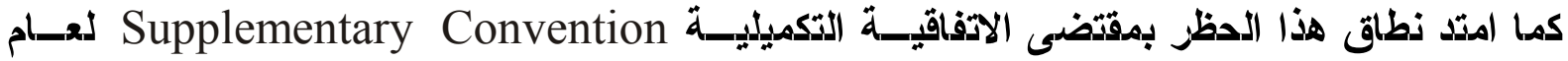

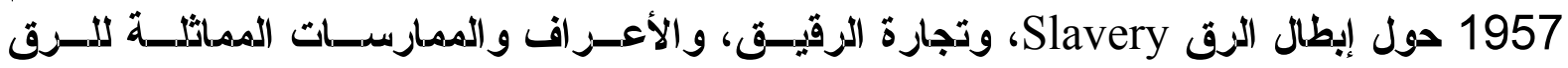

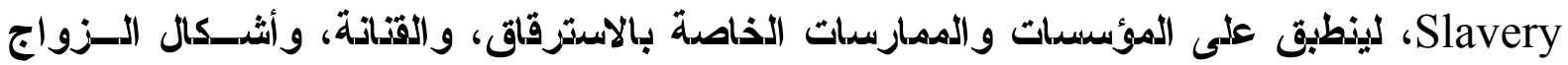

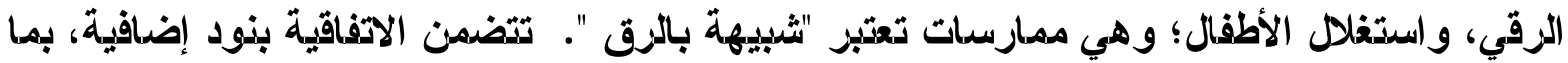

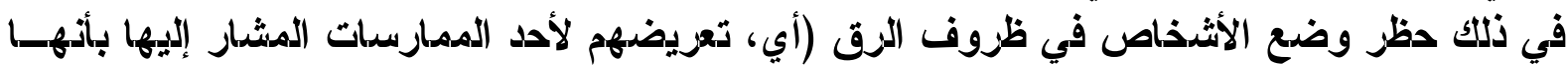

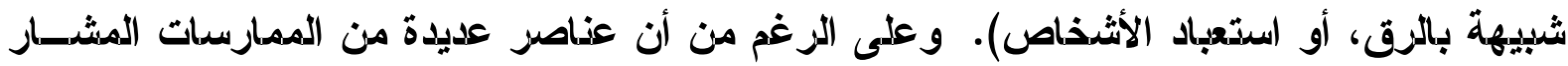

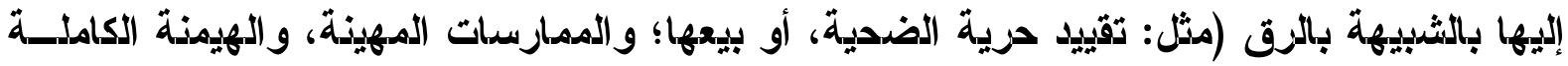

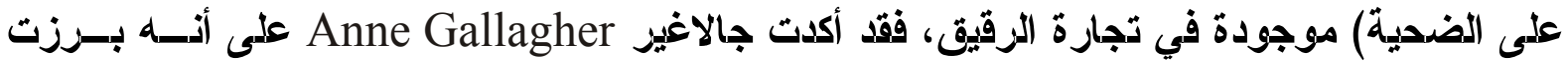

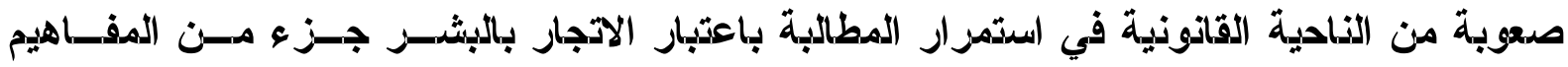
القاتونية التي تحظر الاسترقاق والاتجار بالزقيق.

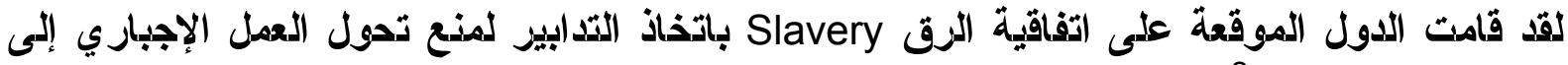

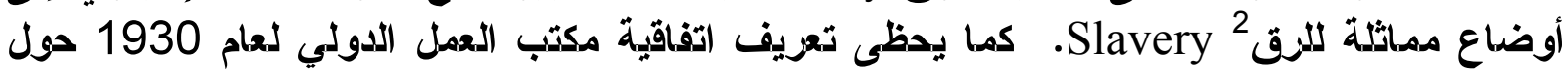

${ }^{2}$ In Gallagher, page 9. Convention on Slavery, Article 5.

Gallagher أنظر صفحة 9 المادة 5 من اتفاقية الثق 


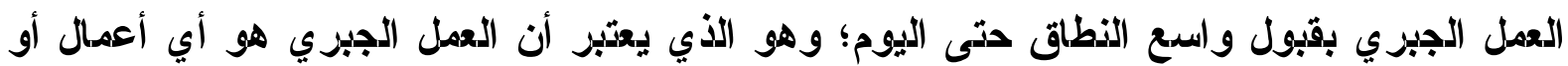

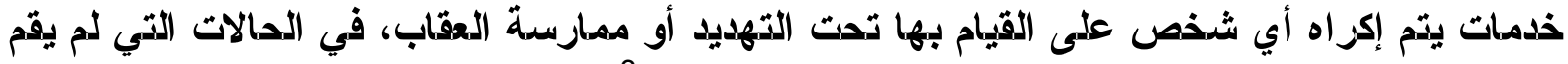

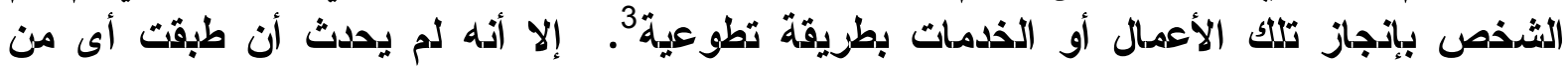

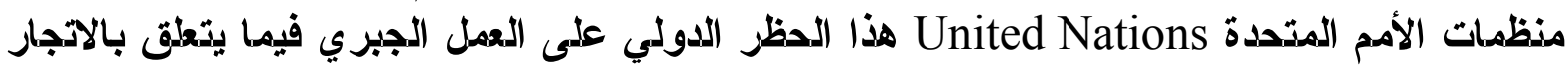

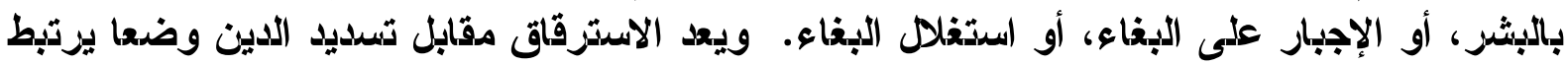

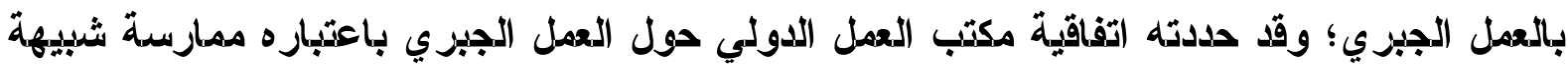

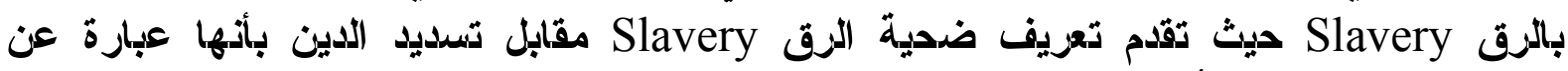
شخص في وضع رقي.

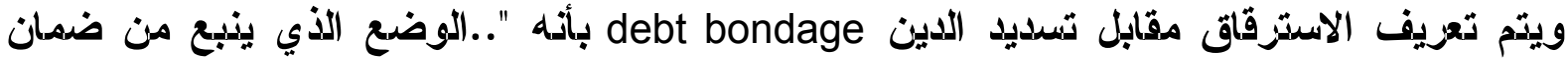

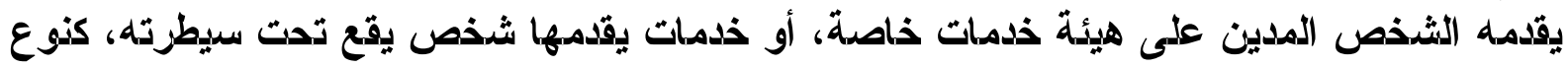

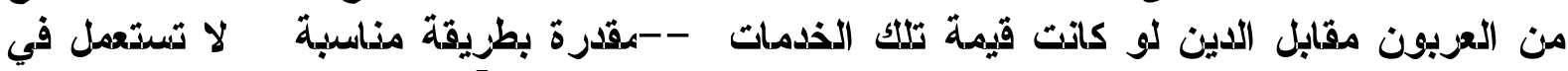

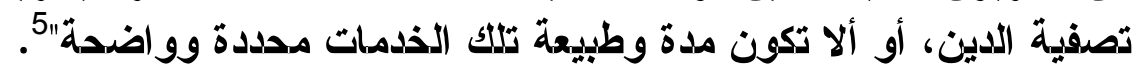

خلال النصف الأول من القزن العشرين، تم إيرام عدة اتفاقيات دولية تتناول الاتجار بالنساء والفتيات

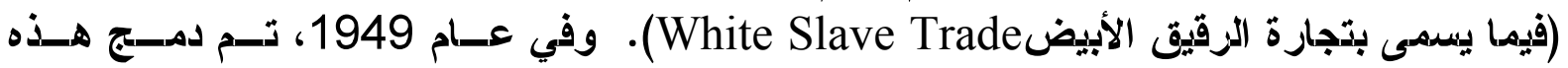

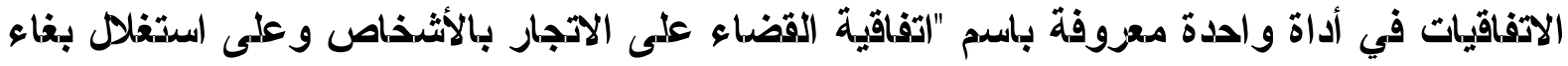

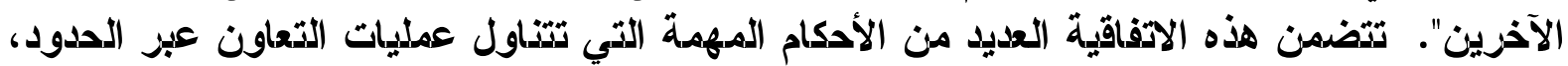

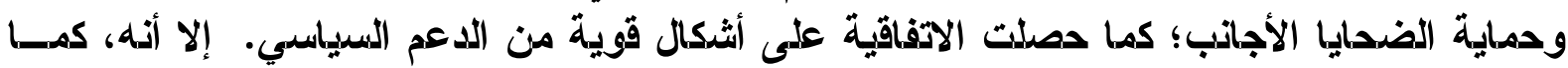

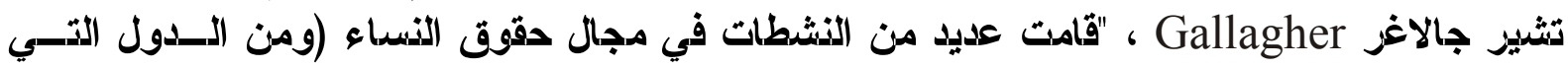

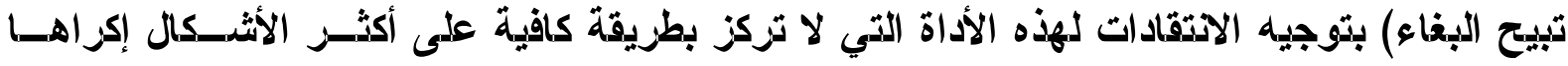

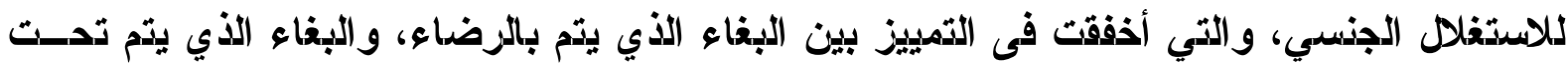

\footnotetext{
${ }^{3}$ In Gallagher, page 9. ILO Convention No. 29 concerning Forced of Compulsory Labor (1939) ف Gallagher
}

صفحة 9 ، اتفاقية منظمة العدل الدولية رقم 29 حول العدل الجبزى أو القهىى (1939)

4 In Gallagher, footnote 100: David Weissbrodt and Anti-Slavery International, Updated Review of the Implementation of and Follow-up to the Conventions on Slavery, Sub-Commission on the Promotion and Protection of Human Rights

فallagher

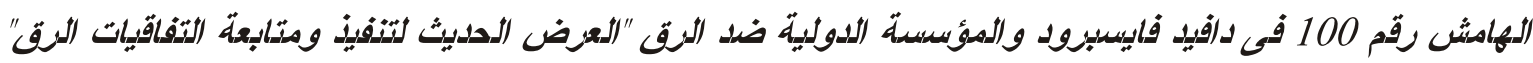
اللجنة الفزعية للنهوض بحقوق الأسان و حمايتها.

${ }^{5}$ In Anne Gallagher, page 10. Supplementary Convention on the Abolition of Slavery, the Slave Trade and Institutions and Practices Similar to Slavery, Article 1(a).

في آن جالاجز صفحة 10. الاتفاقية التكميلية للدد من الثق، و تجارة الدق والمؤسسات والممارسات الشبيهة

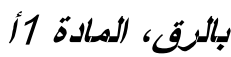




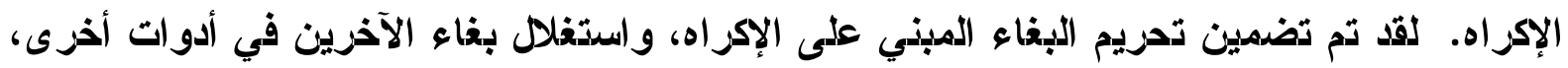
مثل: اتفاقية القضاء على كافة أثدكال التمييز ضد الندين على النساء (Gallagher, p. 9).

وتثظطى اتفاقية حقوق الطفل ما جاء في اتفاقية مكتب العمل الدولي ILO حول الإجبار على العمل؛ الإك

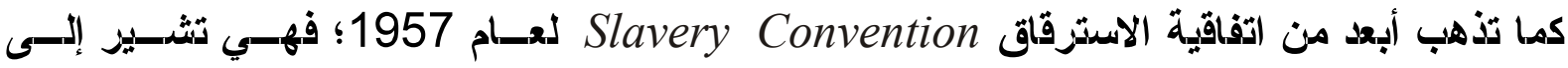

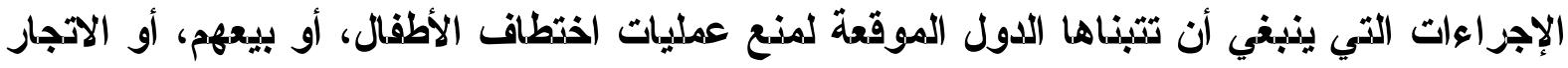

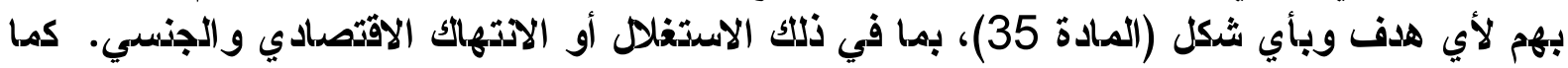

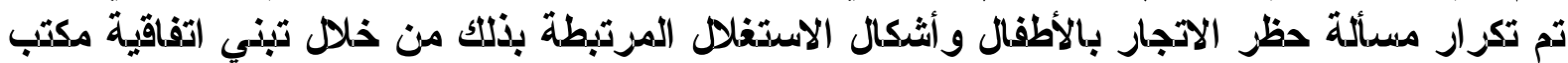

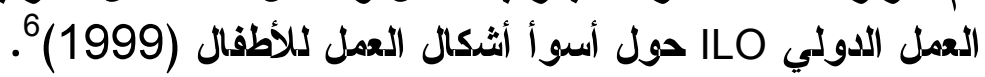

لقد تم استكمال أعمال لجنة الأمم المتحدة حول الجزيمة لعام 1999، واتفاقية نوفمبز 2000

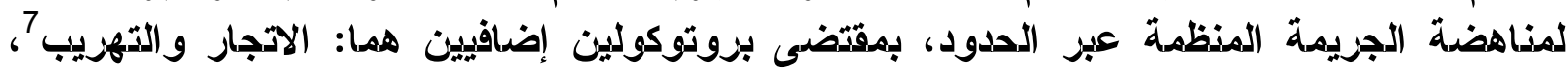

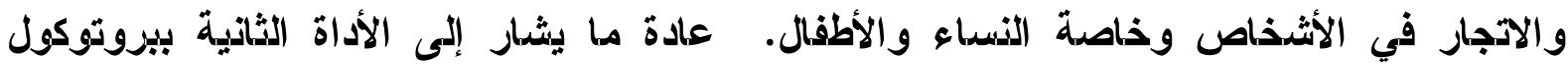

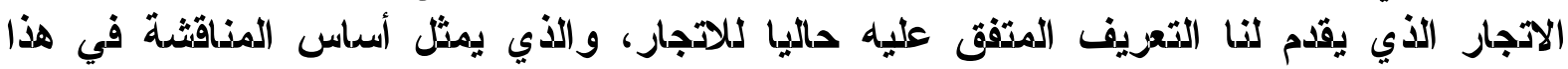

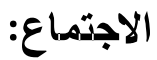

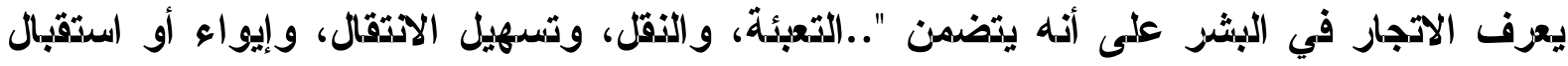

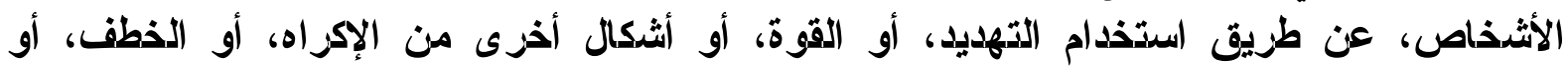

${ }^{6}$ In Gallagher, page 12. Convention Concerning the Prohibition and Immediate Action for the Elimination of the Worst Forms of Child Labor, adopted 17 June 1999 by the General Conference of the International Labor Organization (ILO.)

فى جالاجز صفحة 12، الاتفاقية الذاصة بمنع أسوأ أشكال عمالة الأطفال واتخاذ الاجزاعات الفقرية لإحد منها، تم تبنبيها فى 17 بيونيو 1999 من قبل المؤتمر العام لهنظمة العمل الدولية

7- In Gallagher, page 3. Protocol against the Smuggling of Migrants by Land, Sea and Air, supplementing the UN Convention against Transnational Organized Crime, Report of the Ad Hoc Committee on the Elaboration of a Convention against Transnational Organized Crime on the work of its first to eleventh sessions, UN Doc. A/55/383 (2000), Annex III, Article 3(a). This instrument provides a definition of smuggling that clearly distinguishes it from trafficking: Smuggling of migrants is defined as “... the procurement, in order to obtain directly or indirectly, a financial or other material benefit, of the illegal entry of a person into a State Party of which the person is not a national or a permanent resident."

\footnotetext{
فح جالاجز صفحة 3. البزوتيكيل المناهض لتهزبيب المهاجزين بالبيز والبحر و الجو المكمل لاتفاقية الأمم المتحدة المناهضة

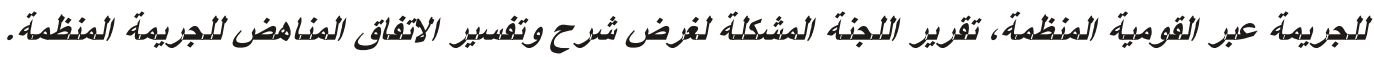
تقدم هذه الآداة تعزيفاً للتهزيب بيميزه بيوضوح عن الاتجار: بيعف تهريب الههاجرين بأنه: "التيوصيل، بهذف الحصول المباشن

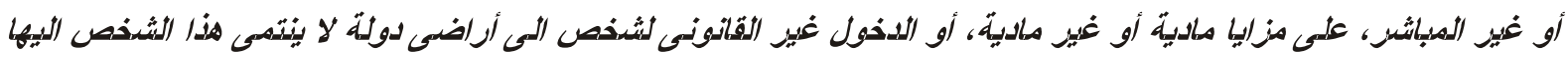
ولايقيم فيها بصفة مستدبيةة.
} 
الاحتيال، أو الذداع، أو استغلال السلطة أو هشاثدة الوضع، أو من خلال الدفع أو تلقي الأموال، أو أو

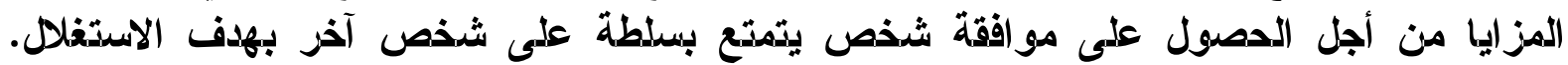

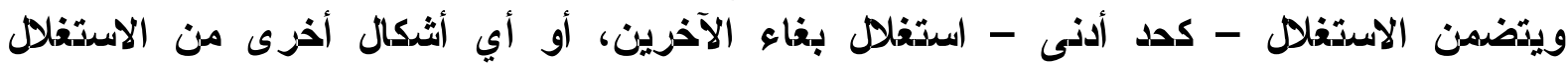

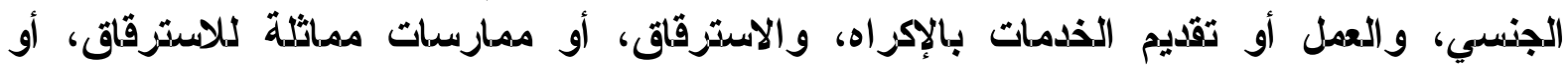

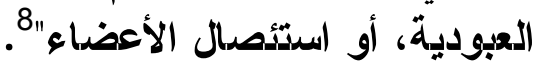

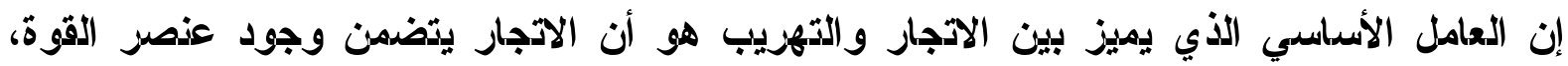

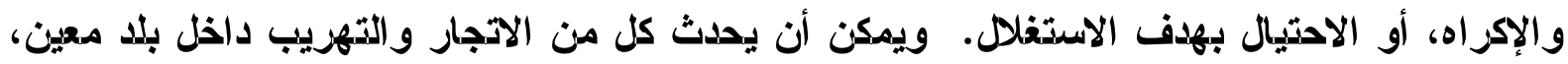

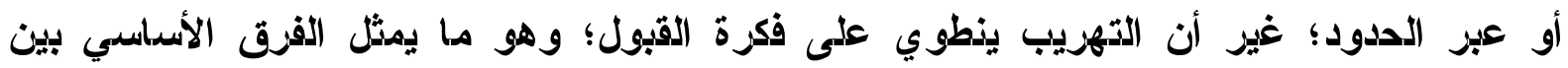

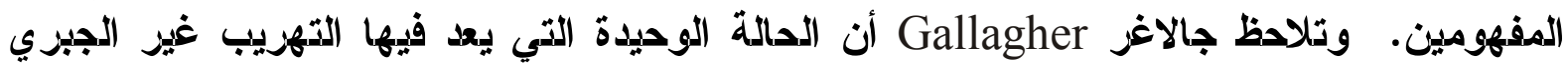

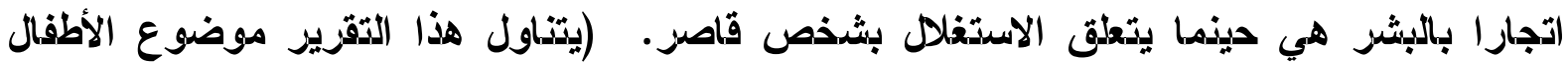

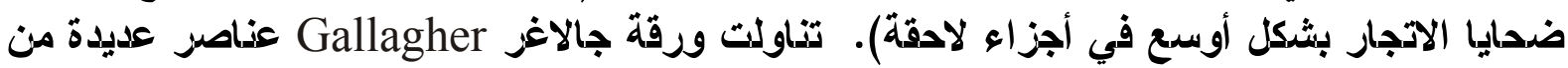

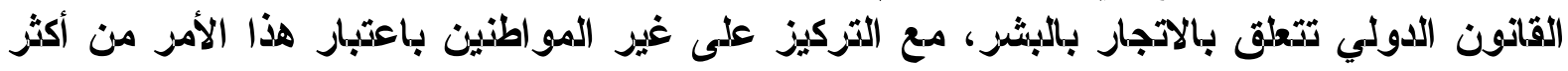

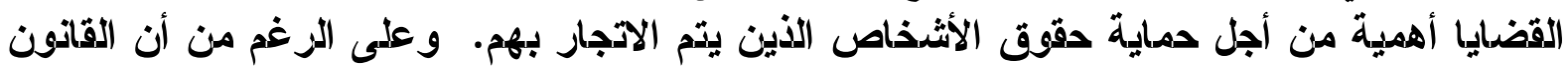

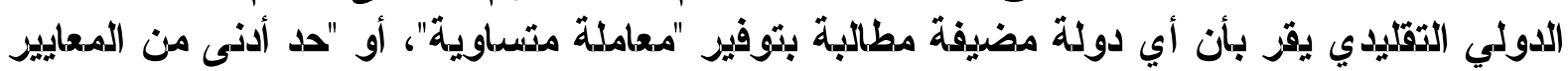

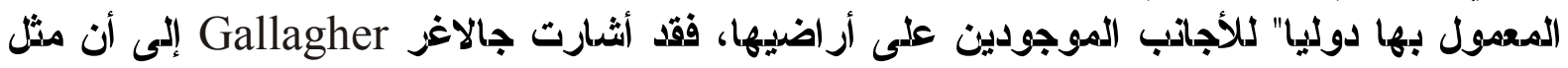

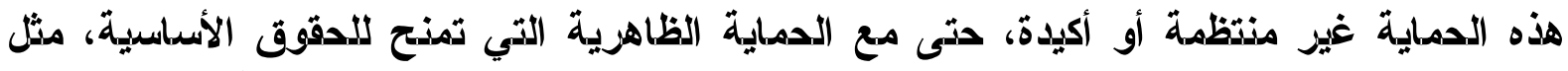

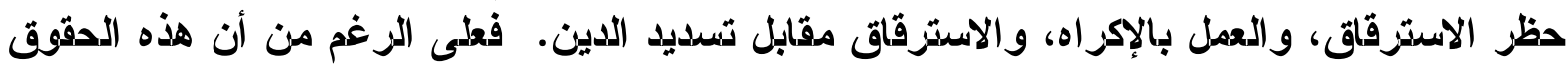

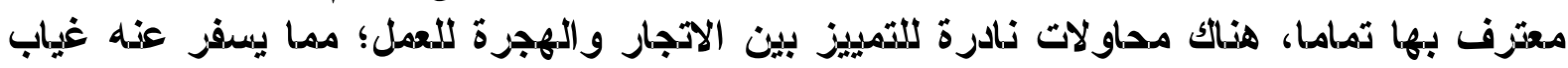

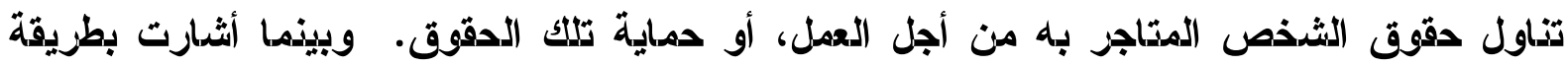

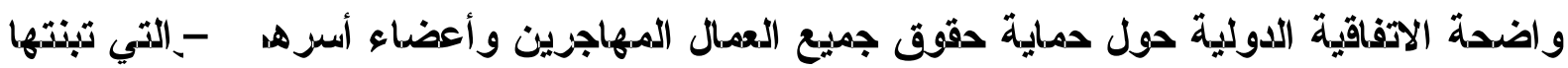

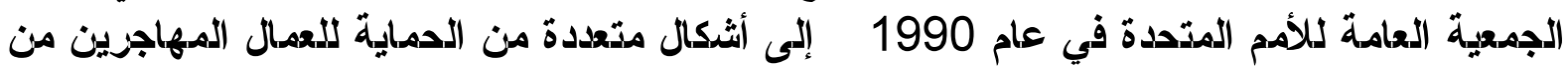

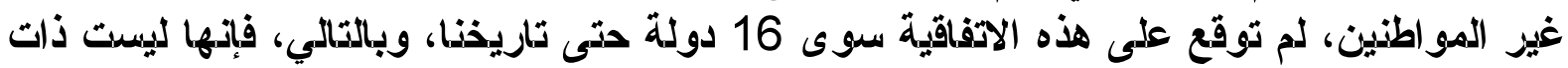

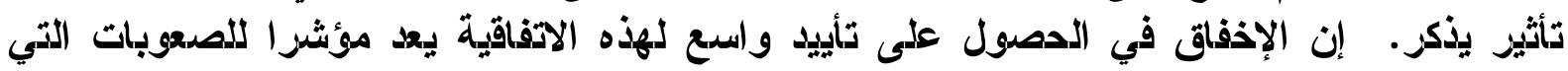
تواجه تأمين الدقوق لغيز المو اطنين.

8 In Gallagher, page 3. Protocol to Prevent, Suppress and Punish Trafficking in Persons, Especially Women and Children, supplementing the UN Convention against Transnational Organized Crime; 12 December 2000, supra note 2, Article 3 (a). The Protocol further states that the recruitment, transportation transfer, harboring or receipt of a child for the purpose of exploitation shall be considered "trafficking in persons" even if this does not involve any of the means set forth in subparagraph (a) Ibid, Article 3(c).

فى جالاجز صفحة 3، بزوتوكول منع معاقبة والدد من الاتجار بالأثذاص ولاسيما النساء والأطفال، المكمل لاتفاق الأمم

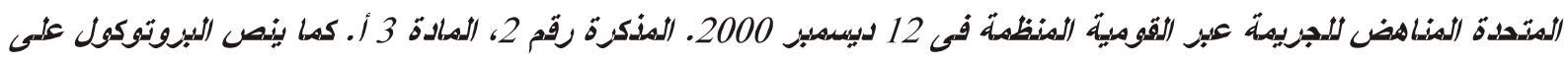

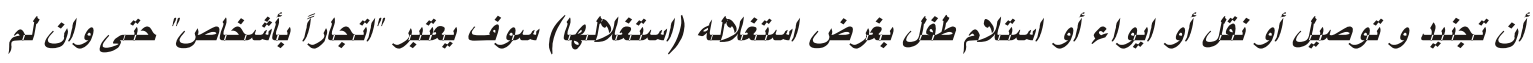

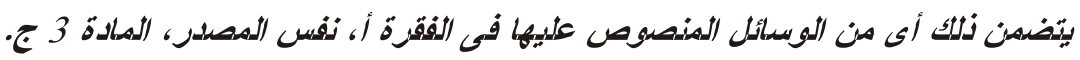




\section{شكل 19}

\section{العركة داخل أو عبر الددود}

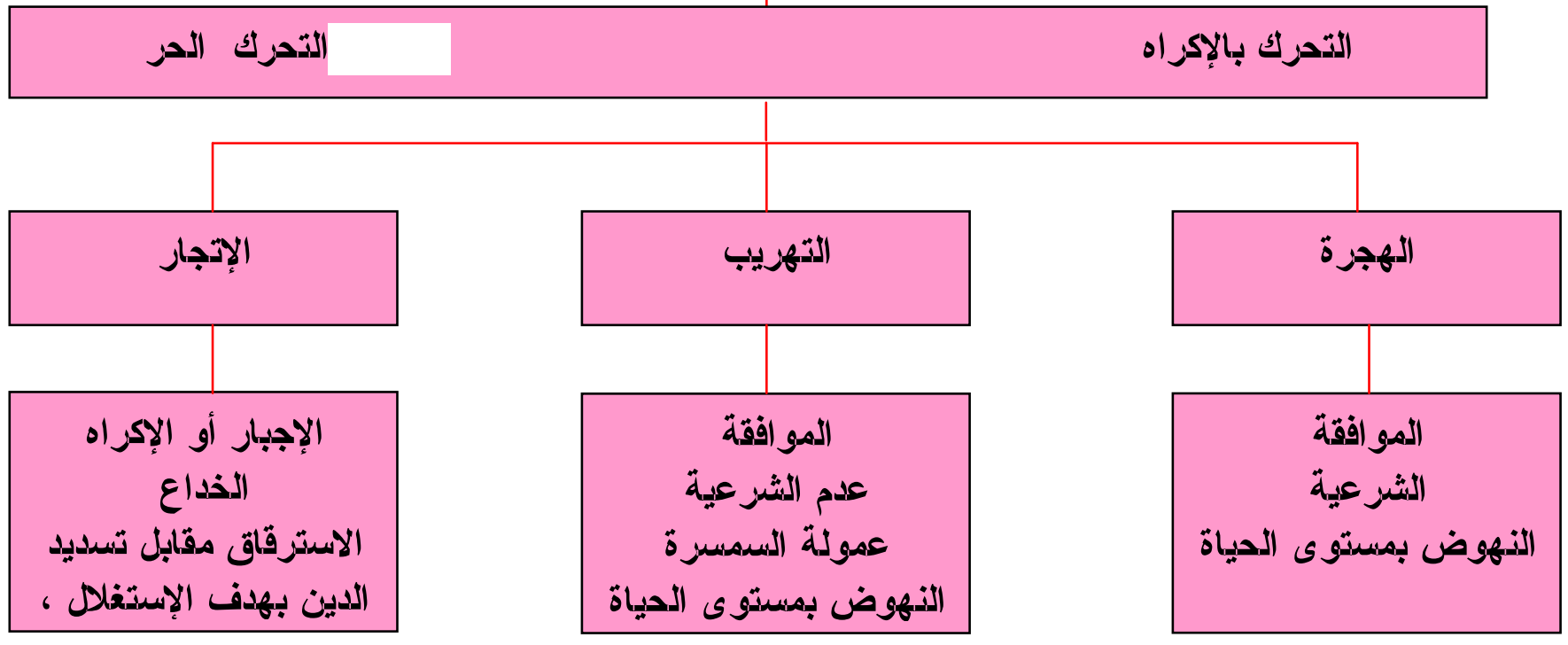

أنهت جالاغز Gallagher مزاجعتها للقانون الدولي المتعلق بالاتجار بملاحظة أن القانون الدولي

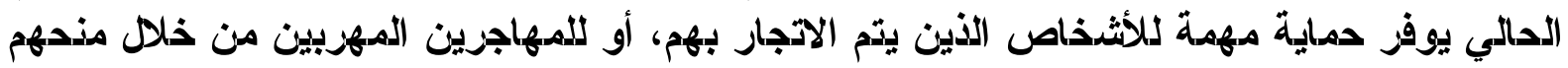

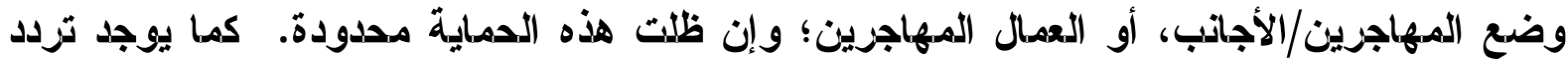

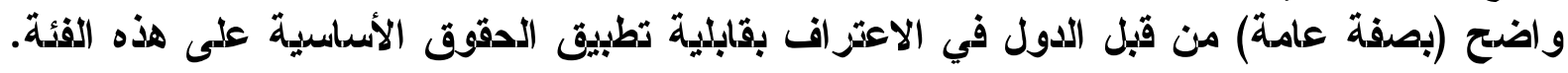

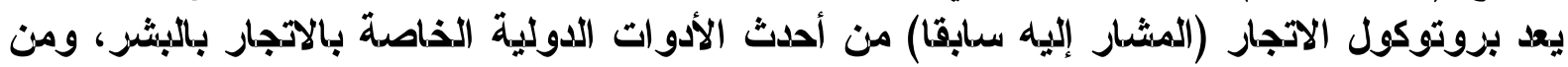

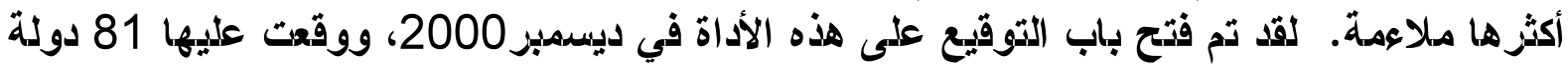

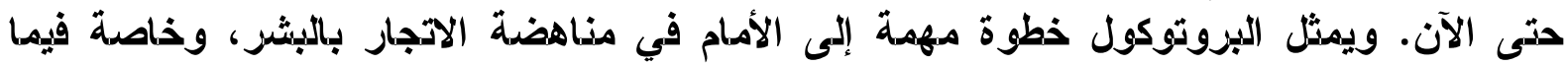

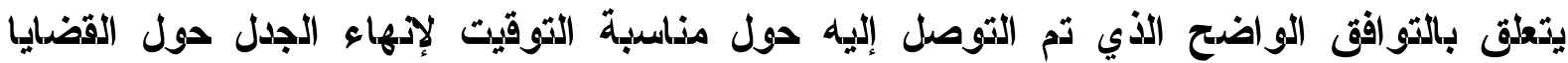

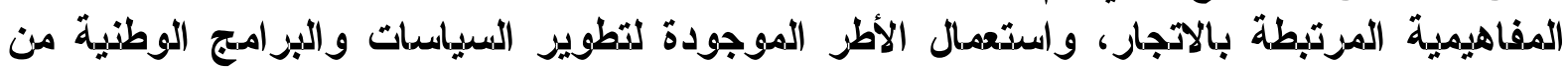

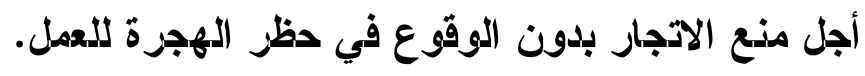

South Asia Region تطبيق الاتفاقيات الدولية في منطقة جنوب آسيا

نتيجة للتواقق حول تعريف الاتجار بالبشر، انتقل الاجتماع إلى الذظر في تطبيق الآدفاقيات الدوليـة

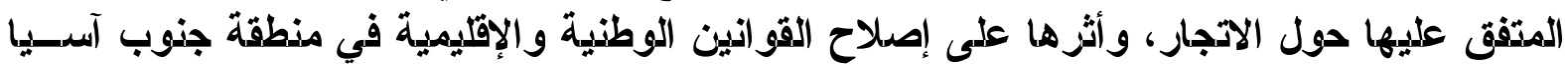

9 Source of Figure 1: Adopted from model presented in Human Rights in Practice Global Alliances Against Trafficking in Women, Bangkok 1999, page 8

مصدر الثكل 1: مقتبس من النموذج المقدم فى حقوق الأسان فى المجال العملى: التدالقات الدولية المناهضة للاتجار بانساء، باتجكو 1999 صفتة 8 
J Jركـز البحسوث القانونيـة Douth Asia Region

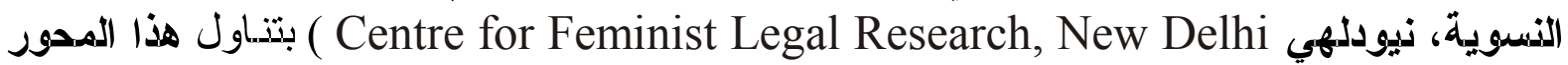

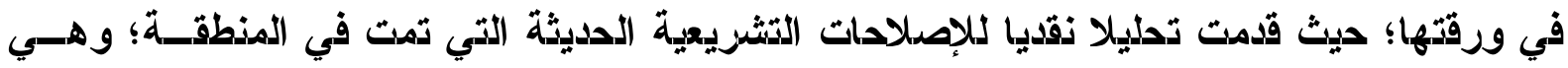

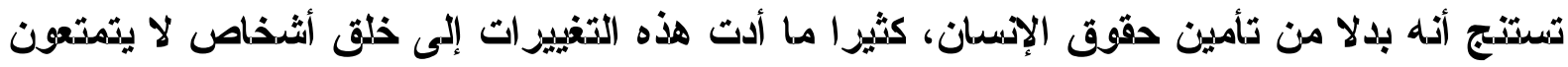

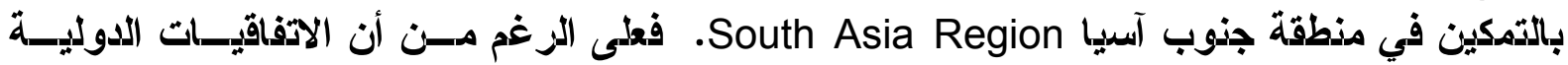

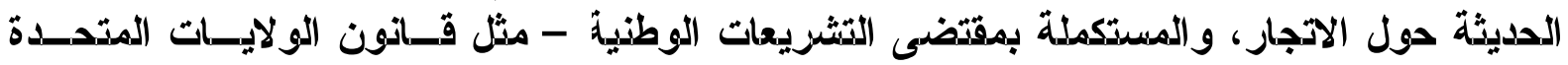

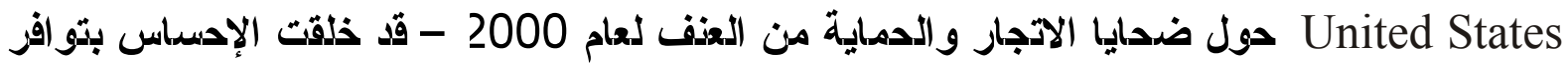

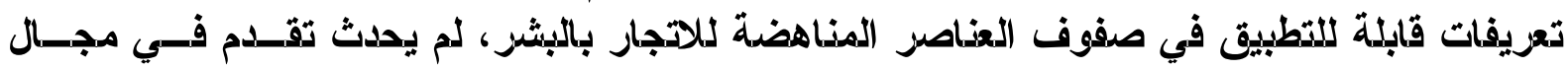

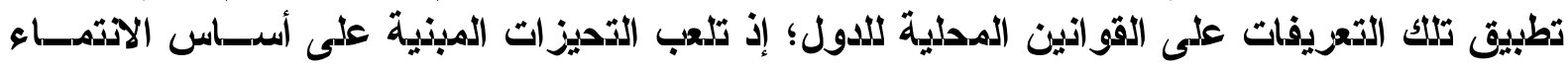

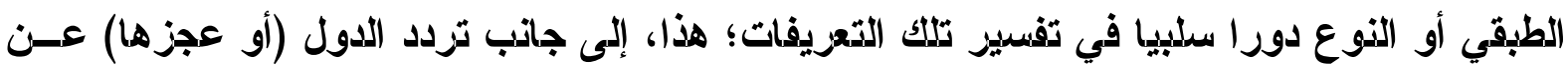

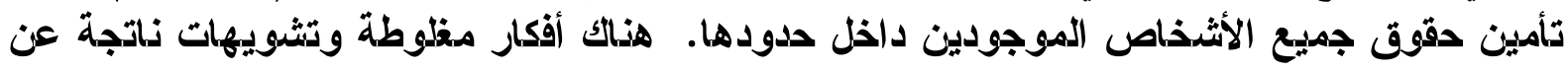

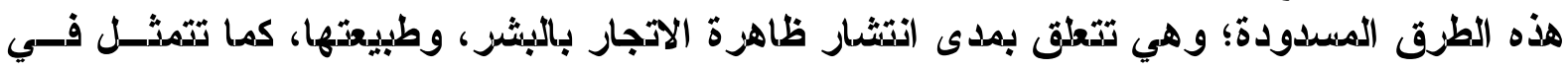

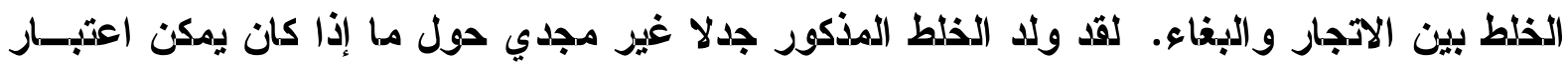

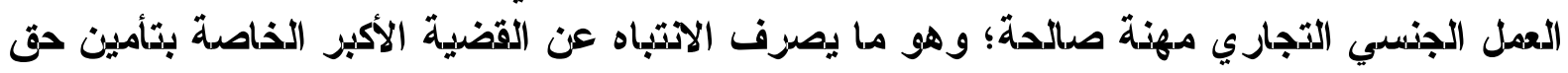

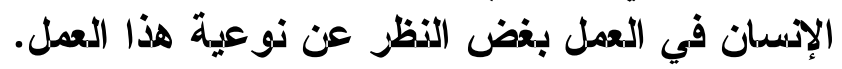

تثيز شاتغيرا Sanghera إلى أن هناك عنصران أساسيان يؤثران سلبيا على التشريعات

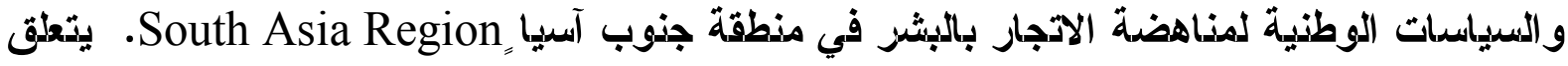

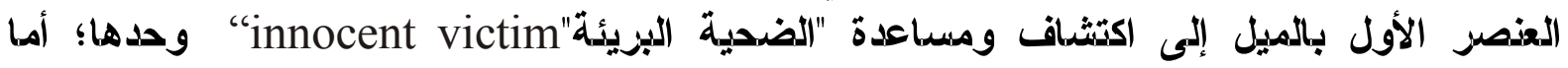

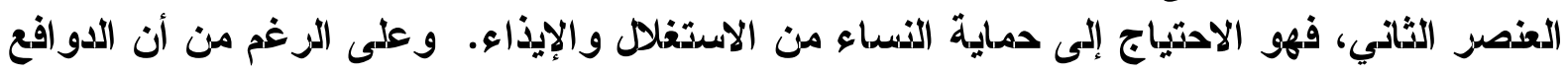

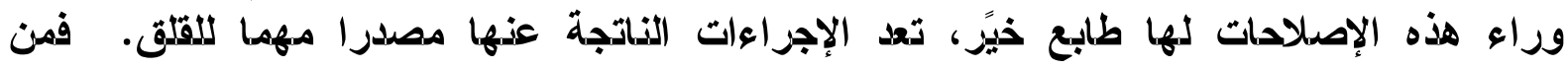

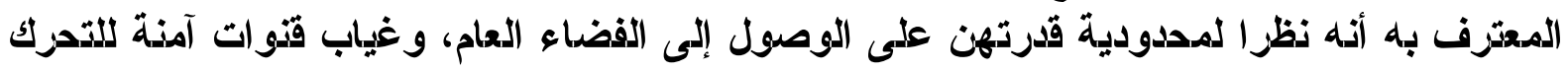

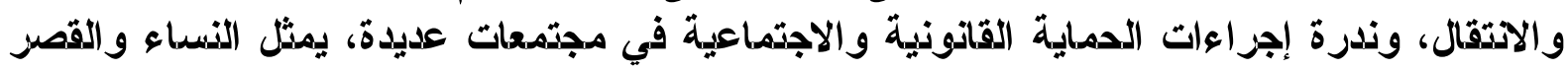

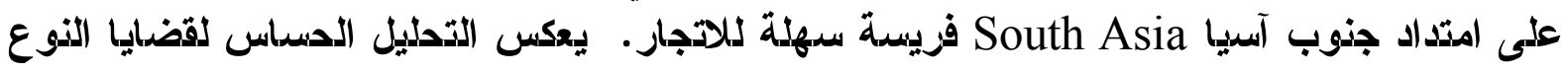

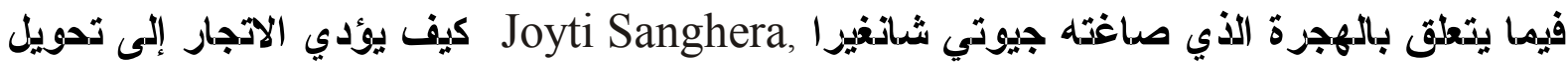

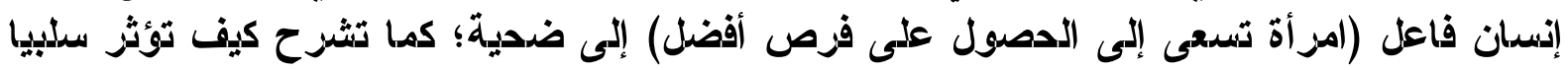

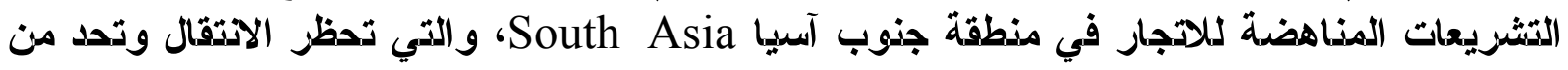

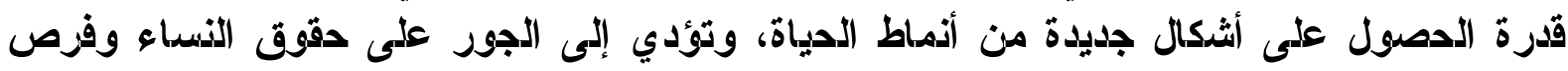

تمكينهن.

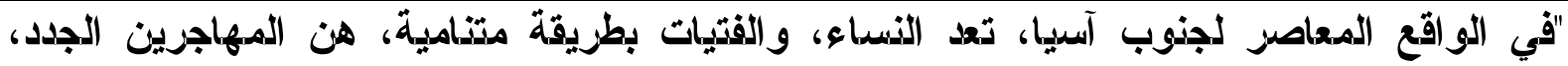

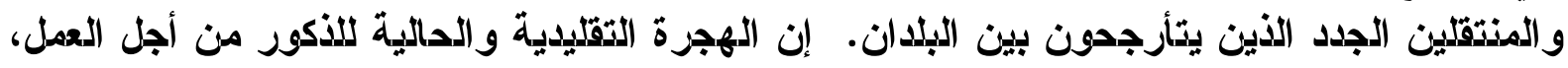

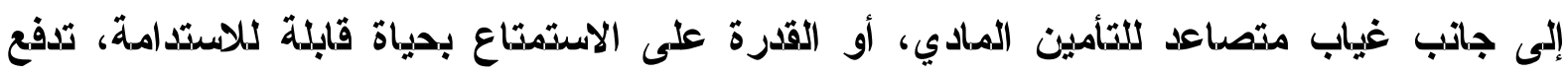

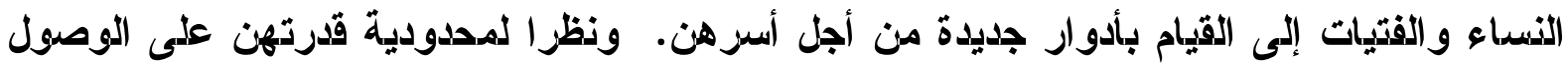

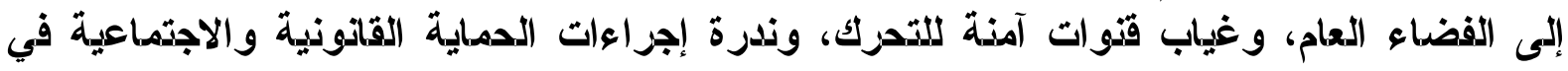

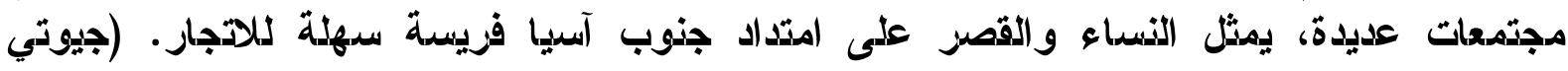

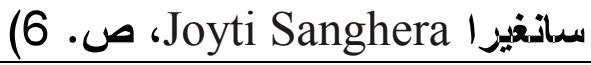




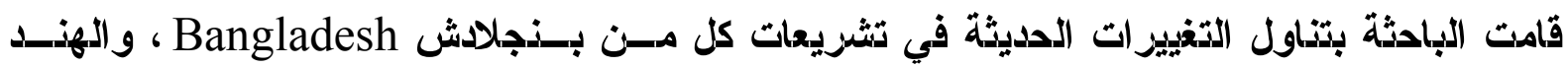
India

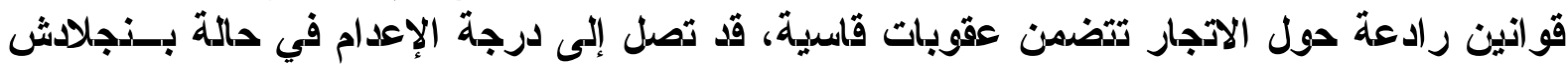

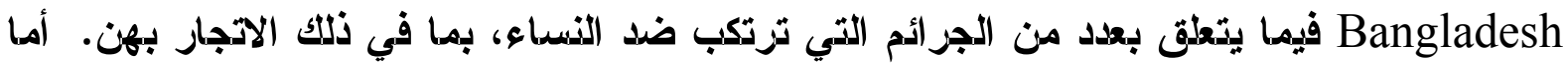

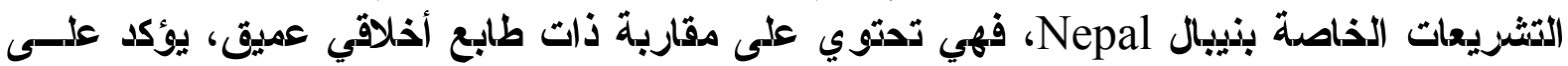

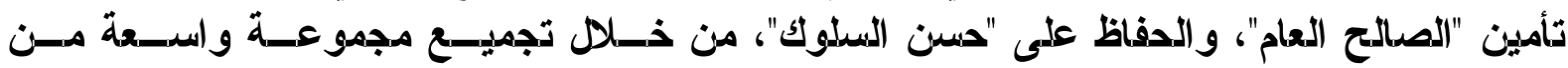

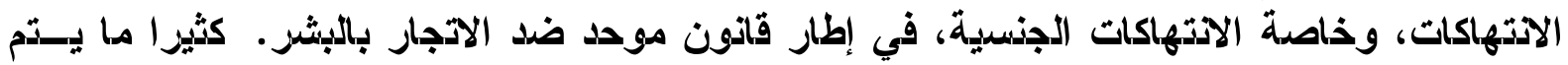

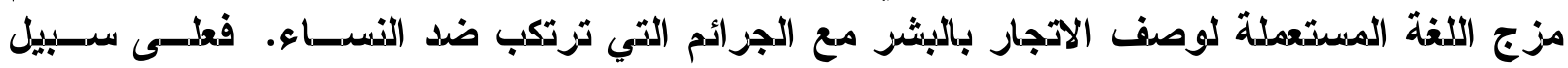

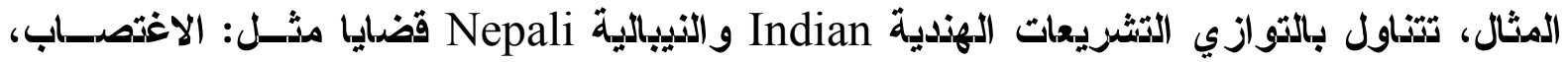

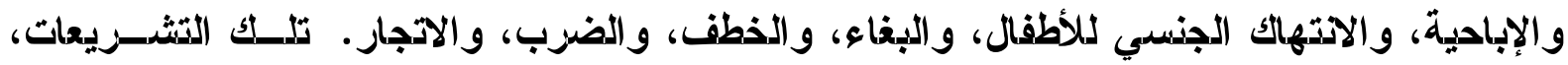

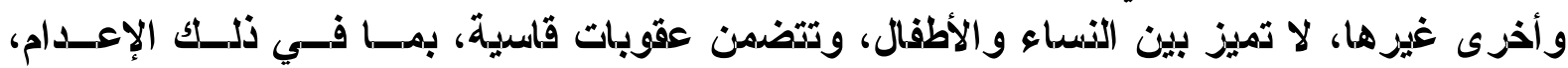

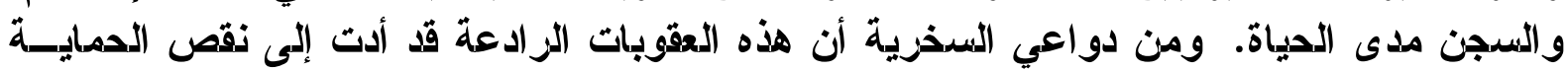

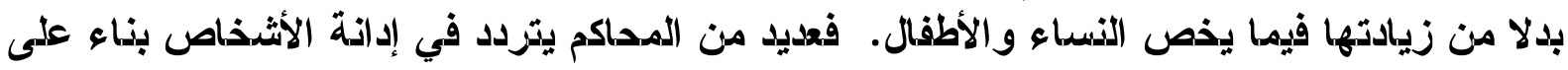

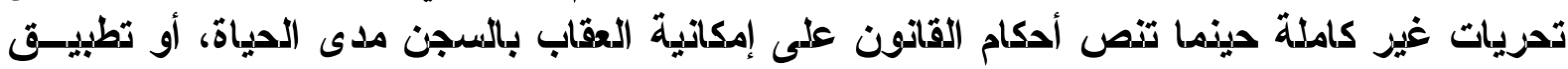

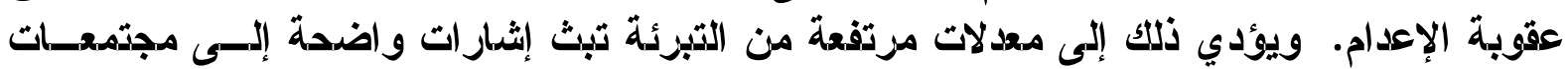

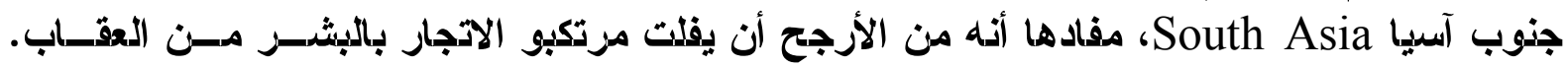

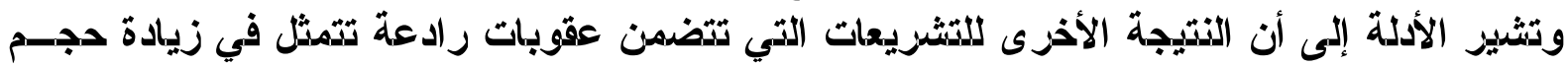

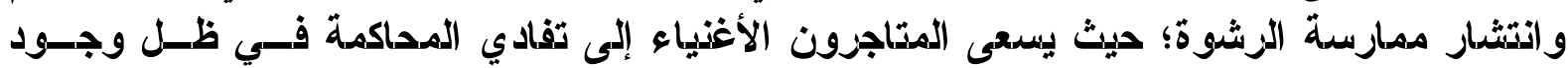
عقوبات كبيزة.

أما الفئة الثانية من القيود القانونية التي تؤدي إلى إضعاف الذهاء، فهي تتمثئل في الإجراءات

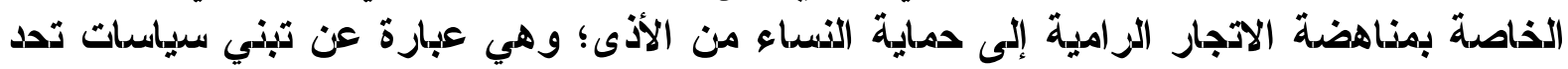

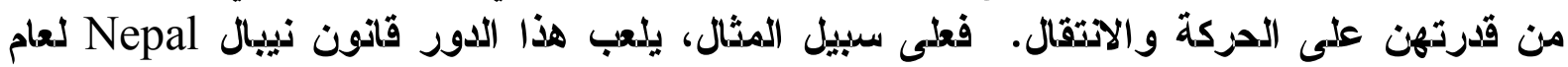

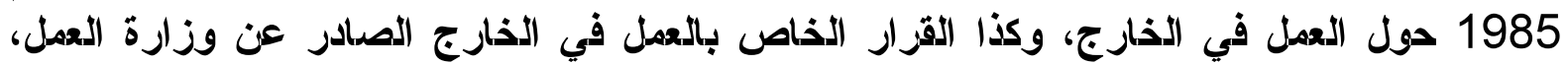

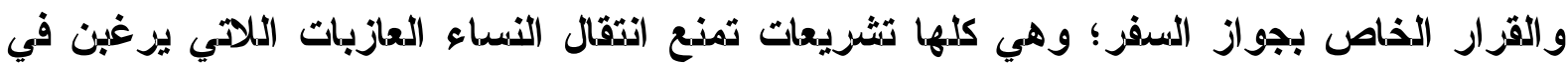

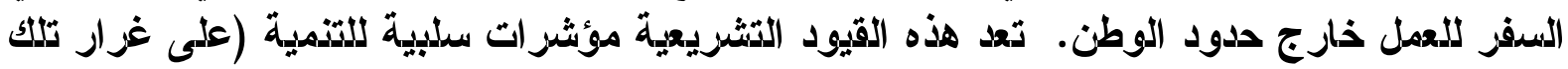

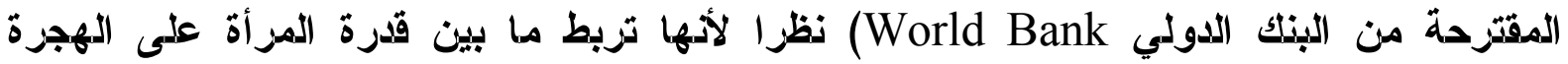
التطوعية بإجزاءات التمكين والتقريز الذاتي.

South أما المؤشر الثالث لإضعاف النساء الناتج عن التثريعات المذكورة في منطقة جنوب آسيا Asia Region

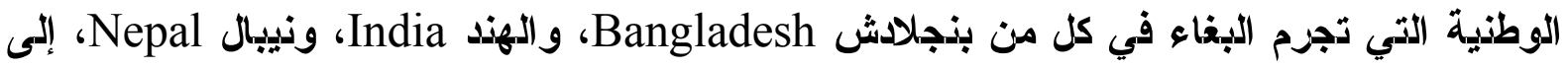

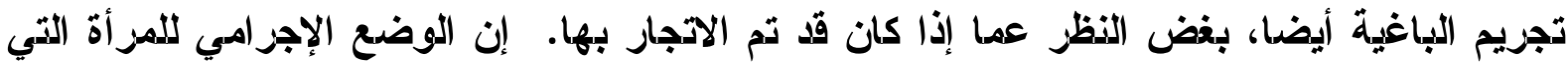

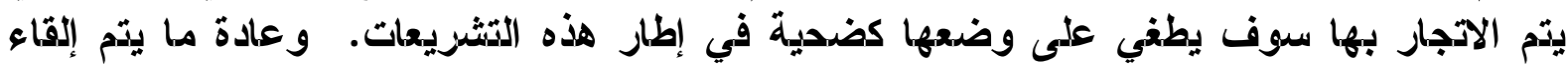

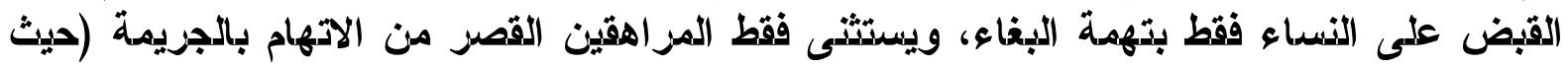

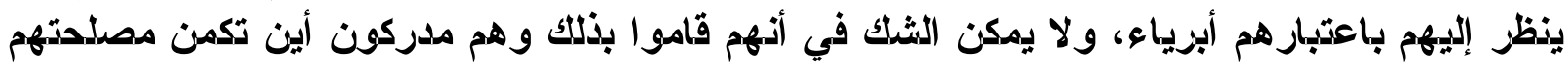

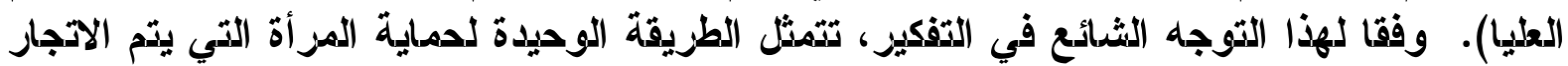

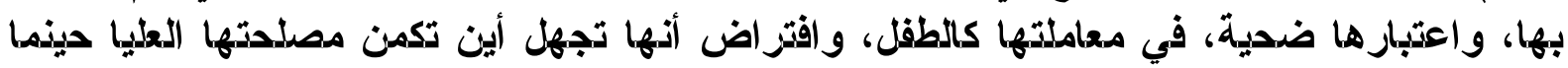


قزرت ممارسة الجنس كمهنة. إن الإدتاج الاجتماعي والقاتوني لضدية الاتجار البريئة عبارة عن

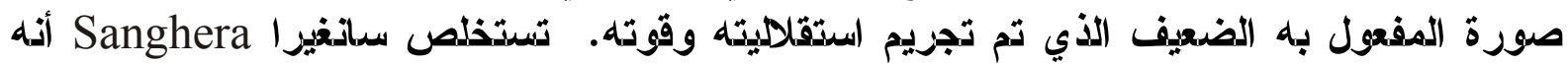

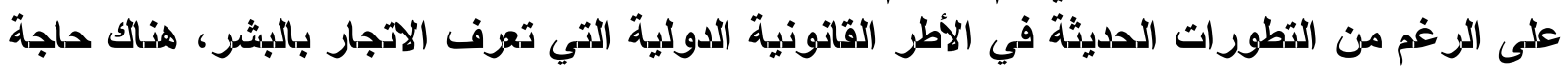

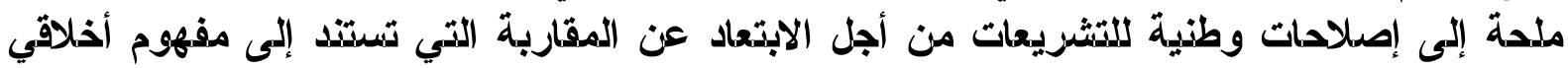

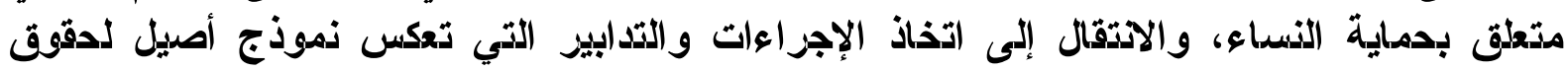
الإسسان، يثمن استقلالية وقوة النساء في منطقة جنوب آسيا South Asia Region.

$$
\text { الاتجار من منظقر حقوق الإسسان }
$$

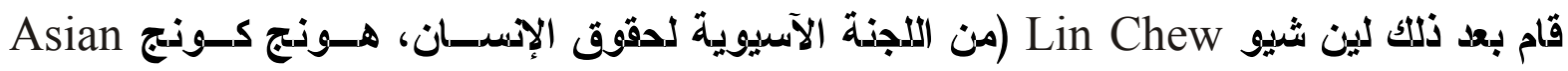
الأجار بالبشن (Commission, Hong Kong Human Rights

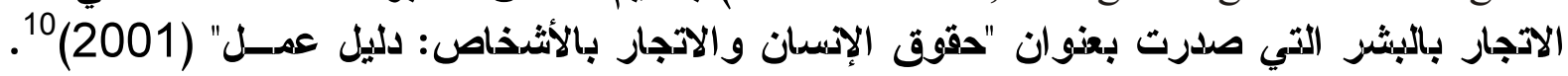

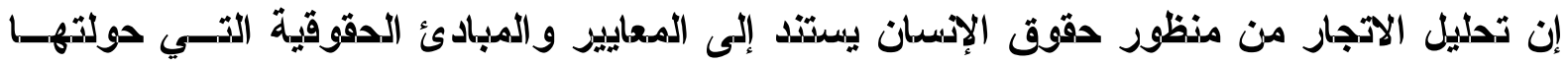

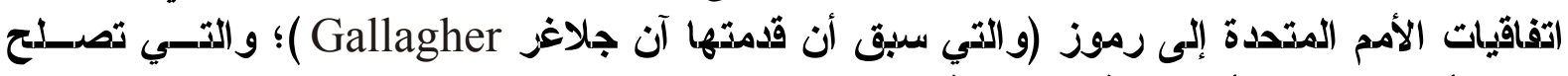
لتكون أساسا لتحليل أي قضية الجتماعية.

بالإضافة إلى هذه الآليات المستتدة إلى اتفاقيات، هناك مكاتب موازية للادفاقيات تابعة لهيئة الأمم

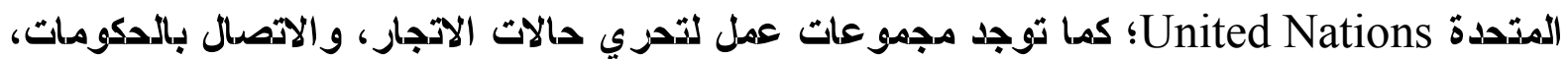

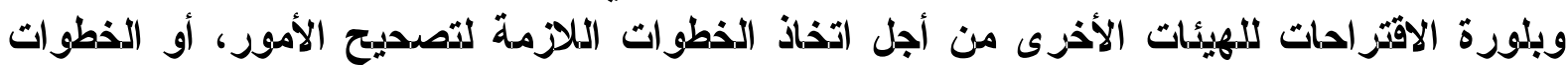

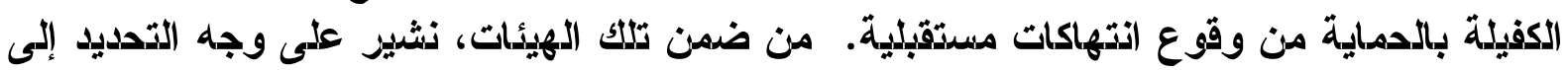

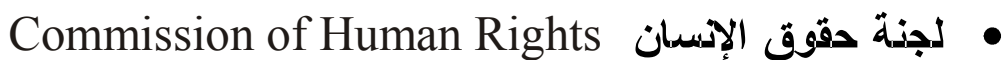

Office of مكتب الدفوض السامي لدقوق الإسسان، والدقزرة الخاصة بالعنف ضد النساء the High Commissioner for Human Rights, Special Rapporteur on Violence against Women المقرر الخاص للدقوق الإسانية للمهاجزين Special Rapporteur on Human Rights of Migrants

Sub-Committee on the Prevention of اللجنة الفزعية لمنع التمييز وحماية الأقليات Discrimination and Protection of Minorities مجموعة العمل حول أثثكال العبودية المعاصرة Working Group on Contemporary مجأ Forms of Slavery

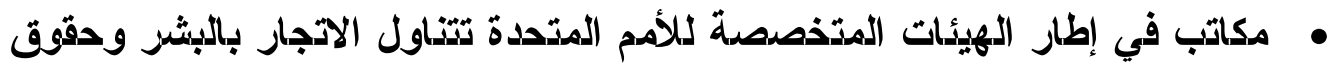

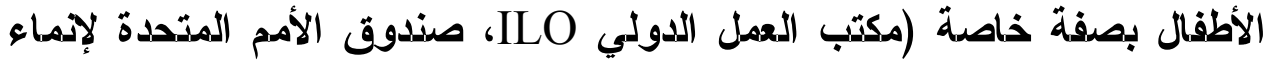

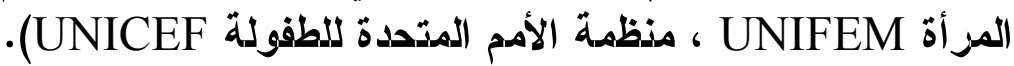

${ }^{10}$ GAATW, Bangkok, Thailand 2001. 
يمكن الذزوج باستنتاج عام بناء على عمل هذه الهيئات الموازية، وهو أن هناتك انتهاكات شديدة

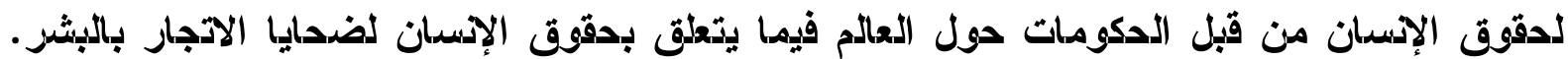

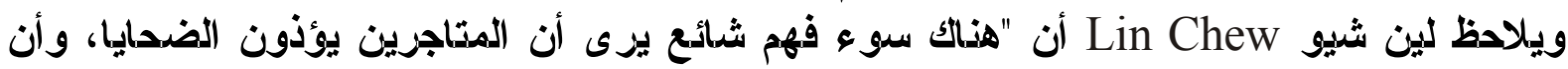

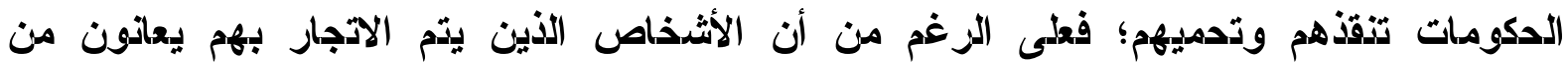

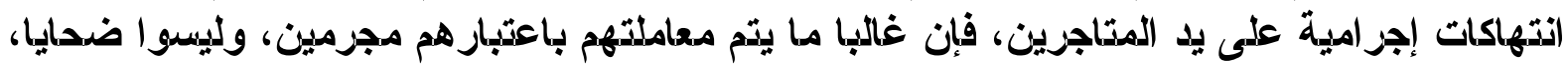

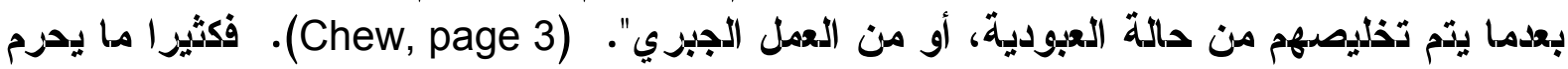

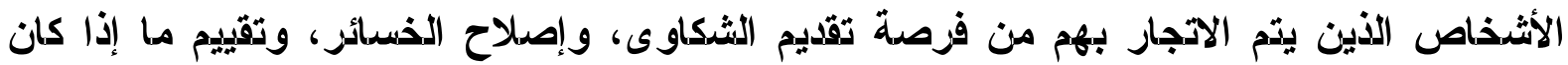

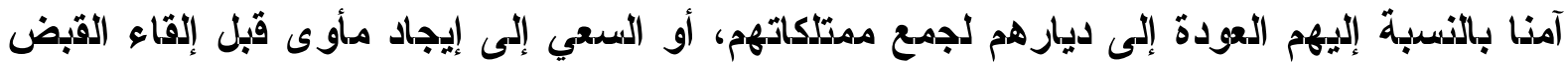

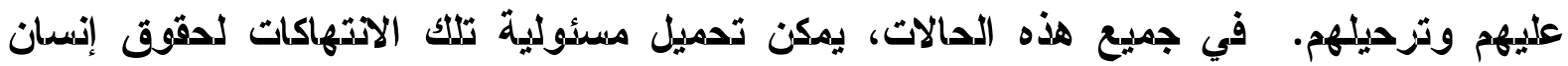

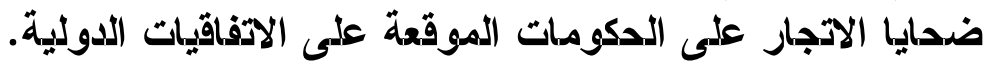

شدل 211: انتهاكات حقوق الإسدان في عملية الاتجار

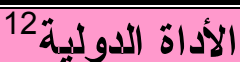

الدقوق

الآتهاكات الإجزامية

11 Source: Human Rights and Trafficking in Women: A Handbook, GAATW, Bangkok, 2001. cited in Lin Chew.

المصدر: حقوق الاسنن والاتجار بالنساء: دليل، باتجوك 2001، كما ورد فى مين تثو

12 List of abbreviations of international instruments:

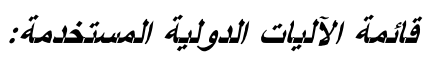

UDHR: Universal Declaration of Human Rights ICCPR: International Convention on Civil and Political Rights

الاتفاق العالمس لدقوق الاسنان

الاتفاقية الدولية للدققق المدنية و السياسية

ICESCR: International Convention on Economic, Social and Cultural Rights

الاتفاقية الدولية للدقوق الاقتصادية و الاجتماعية و الثقافية

CAT: Convention against Torture and Other Cruel, Inhuman or Degrading Treatment or Punishment الآفقاقية المناهضة للتعذيب والمعاملة القاسية أو غير الآدمية أو المذلة أو العقاب

CEDAW: Convention on the Elimination of All Forms of Discrimination against Women

اتفاقية الدد من كافة أشكال التمييز ضد المرأة

CRC: Convention on the Rights of the Child

اتفاقية حقوق الطفل

ILO No. 29: International Labor Organization Convention No. 29 Concerning Forced Labor اتفاقية منظمة العمل الدولية رقم 29 حول العمل القسرى

ILO No105: International Labor Organization. Convention No. 105 Concerning Abolition of Forced 


\begin{tabular}{|c|c|c|}
\hline الأداة الدولية2 & الحقوق & ت الإجزامية \\
\hline 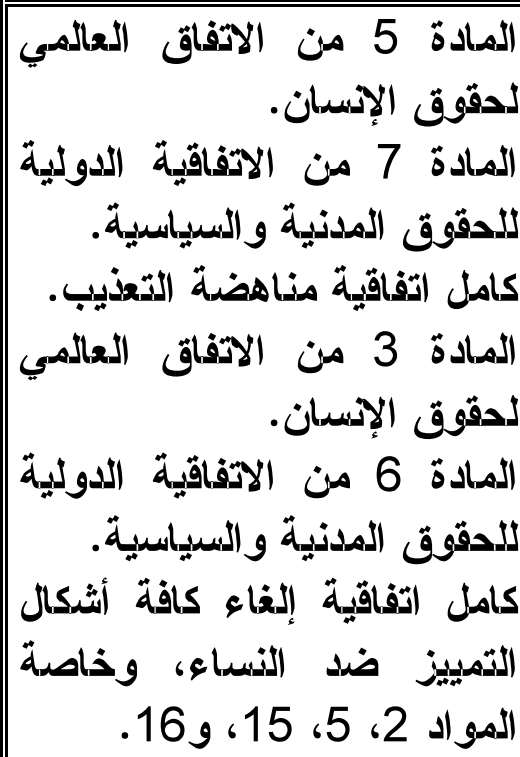 & 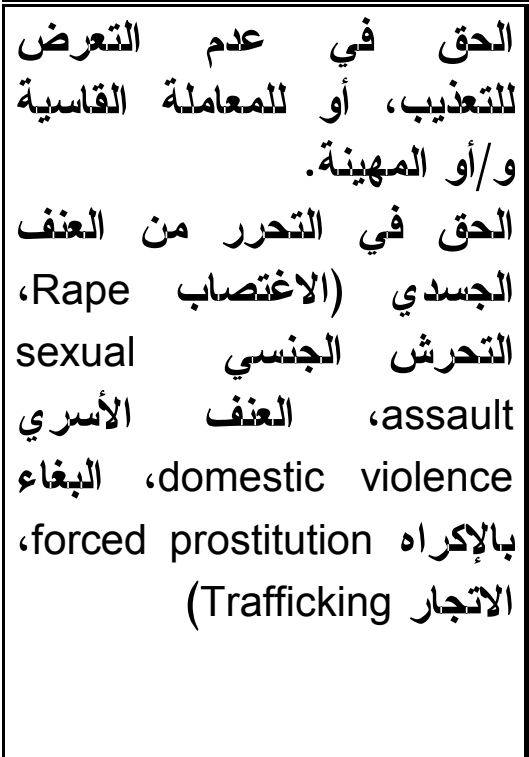 & 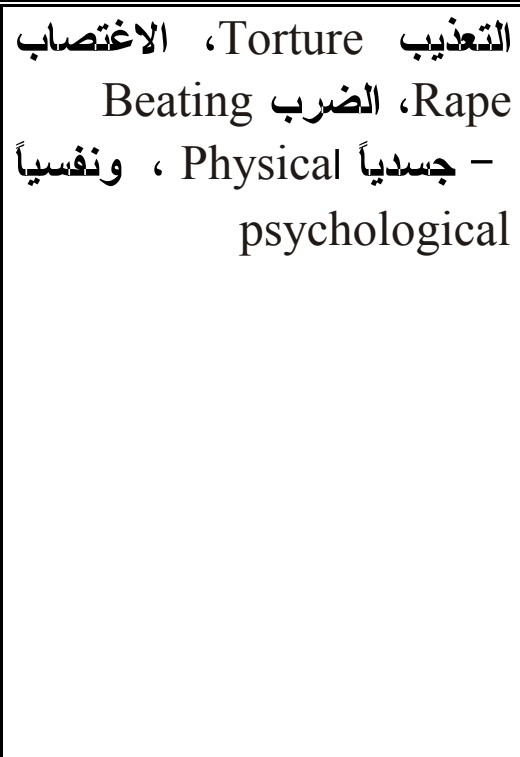 \\
\hline 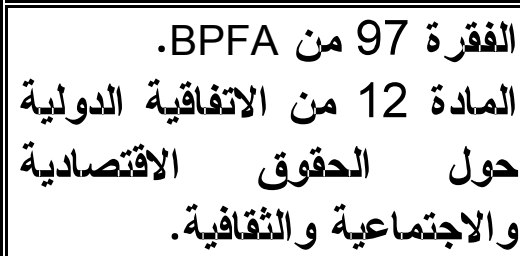 & 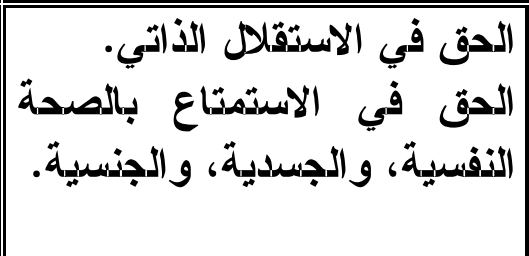 & بالإكران \\
\hline لالدقادة الإسسان. 12 من الاتفاق العالمي & الذاتي. & الأسرة عذد بالادتقام من الإلى أعضاء \\
\hline 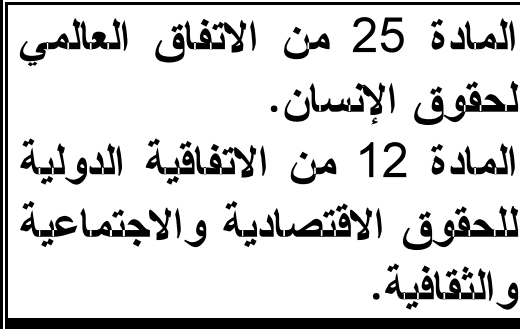 & |الذفسية، وي الجسدية، والجتاع بالصدية. & 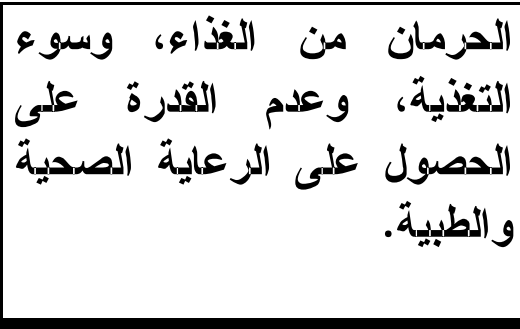 \\
\hline 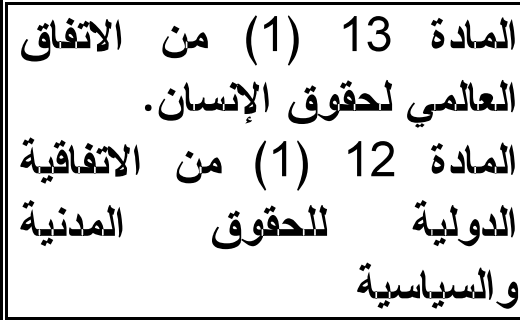 & ورالادية الختل داخل البلد. مدل الإقامة، & 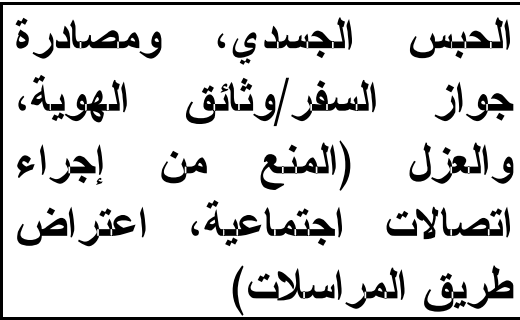 \\
\hline
\end{tabular}

Labor.

اتفاقية منظمة العمل الدولية رقم 105 الذاصة بالحد من العمل القسزى

UNSC: United Nations Slavery Convention

الثفاقية الأمم المتحدة المناهضة للزق

UNSCAS: Supplementary Convention on the Abolition of Slavery, the Slave Trade and Institutions الاتفاق التكميلى دول الد من الدق وتجارة الرق ومؤسساته 


\begin{tabular}{|c|c|c|}
\hline الأداة الدولية2 & الحقوق & الآتهاكات الإجزامية \\
\hline 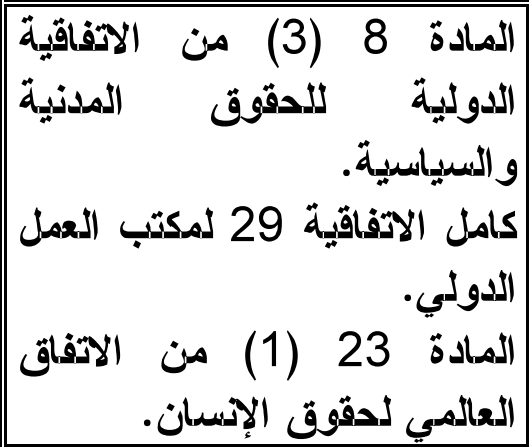 & 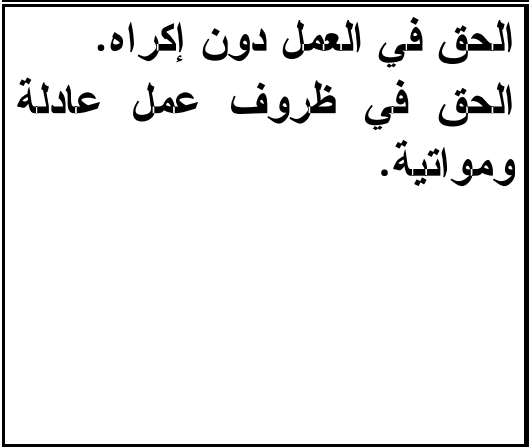 & الدمل الدصول على سائد، راحة طويلة، \\
\hline 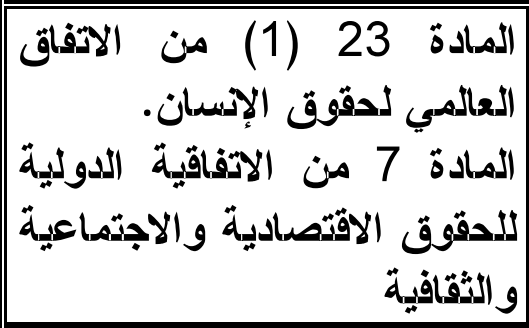 & |آمنة. في ظزوف عمل صدية & صدية وأمنية ضعليفة سيئة، إجزاعات \\
\hline 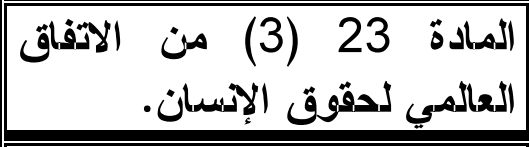 & |الحق في أجز عادل ومناسب. & |لعياب أو تأجيل دفع الأجز مقابل \\
\hline |للدقوق المدنية والديادة 11 من الآفاقية الدولية & 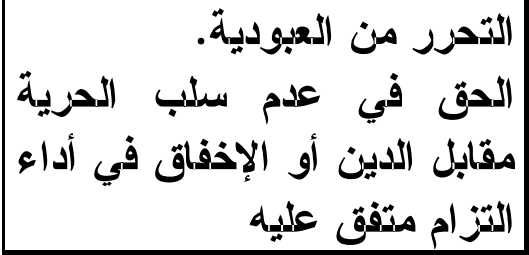 & مقابل تسديد الدوسين، والاسترقاق \\
\hline |العادمي لدقوق 23 (2) من الإدسان. الآفات & |لالعدل المتسي الأجز المتساوي مقابل & العمل التهاك (لعقد من قبل صاحب| \\
\hline
\end{tabular}

يتضمن بروتوكول الاتجار بالبشز Trafficking Protocol مواد قوية قابلــة للتطبيـق قانونيـانيا،

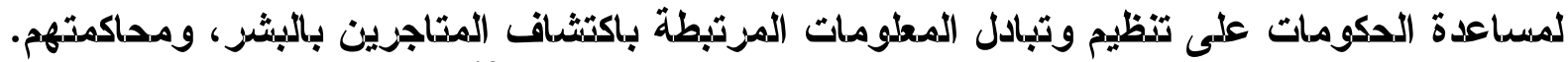
وفي حين يتسم تركيز بزوتوكول الآجار

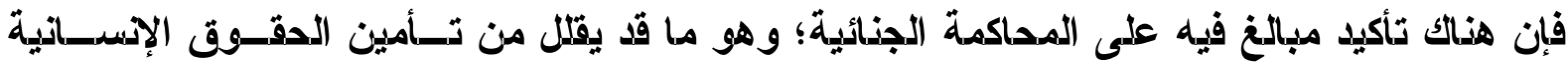

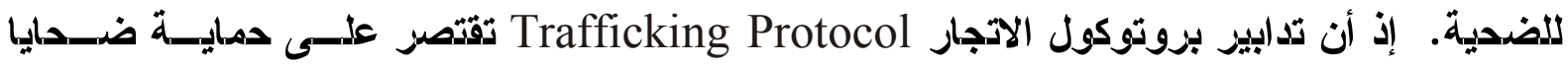

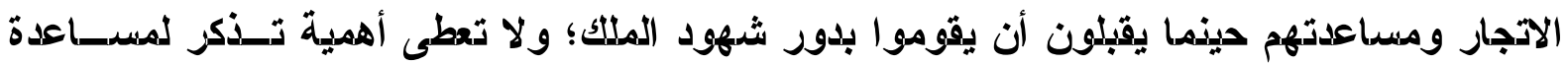

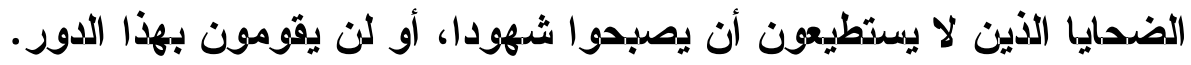

دفعت هذه الذواقص لين شيو Lin Chew إلى تسمية تعريف الاتجار الموجسود فــي البروتوكسول

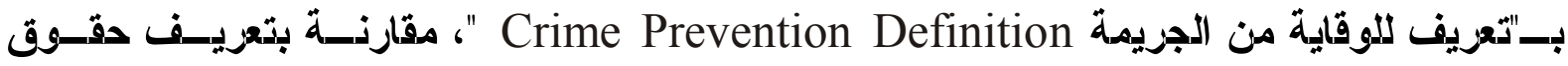

${ }^{13}$ UN Special Rapporteur on Violence against Women Radhika Coomaraswamy; Report to the UN Commission on Human Rights (E/CN 4/2000/68), February 2000. (in Lin Chew, page 6)

المقرد الذاص حول العنف ضد النساء راديكا كوماراسوامى، تقزيز مقدم للجنة الأمم المتحدة عن حقق الأسان (فبرايز

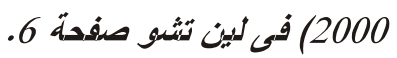


UN Special Rapporteur الإسسان الذي تبنته مقزرة الأمم المتحدة الخاصة بالعنف ضد الذهاء الذهاء UN on Violence against Women :Commission on Human Rights

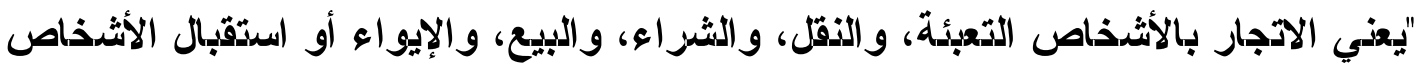

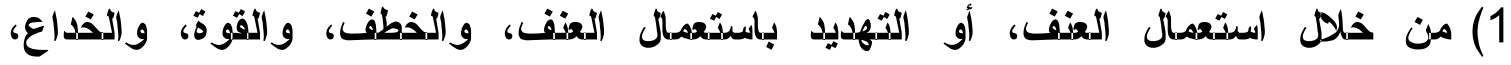

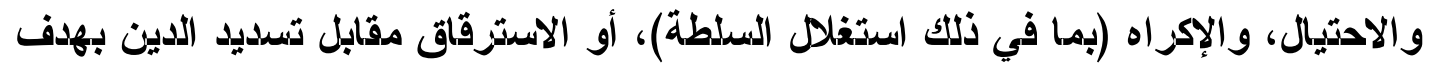

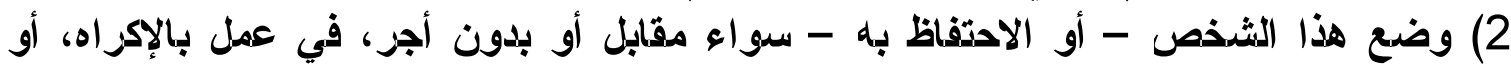

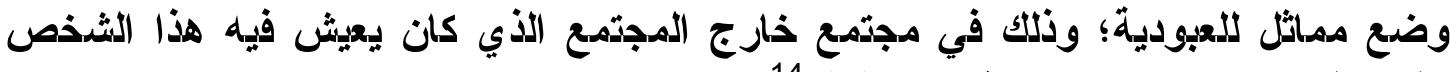

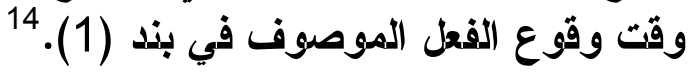

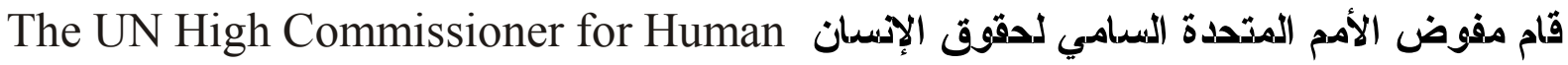

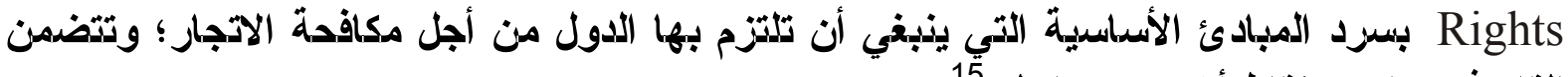

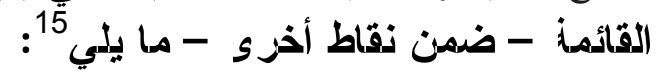

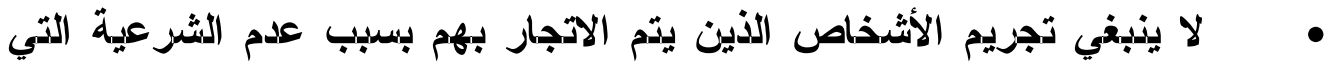

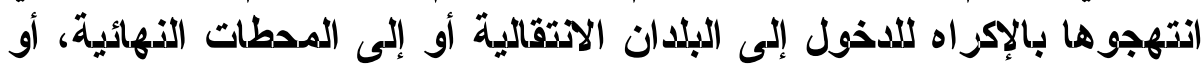

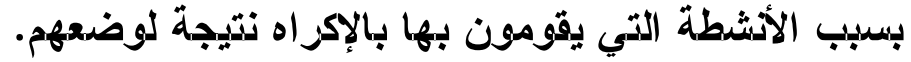

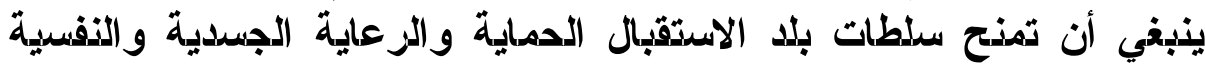

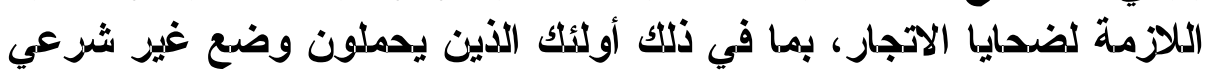
ل اللهجزة. ينبغي تأمين العودة الآمنة للضدايا بدلا من الترحيل التلقائي، خاصة في

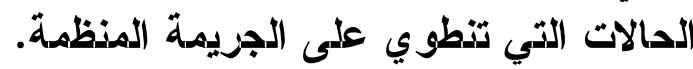

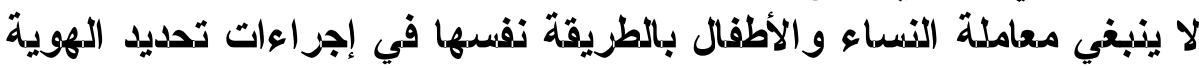

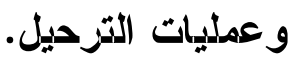
يجب بذل الجهود لتناول الأسباب الجذرية للاتجار بالبشز، بما في ذلك الفقر، وعدم المساواة، و التمييز، و الإنصرية.

تمثل الدقوق مبدأ مجزد يحتاج إلى التحديث من خلال تطبيق واع لمبادئ مثل تلك التي وصفها

${ }^{14}$ Mrs. Mary Robinson, UN High Commissioner for Human Rights, address to the International Abolitionists' Federation Conference on Trafficking in Women "Breakdown of Borders," Copenhagen, December 2, 1999 (Cited in Chew, page 9).

السيلة مارى روينسون، مفوضة الأمم المتددة لدقوق الاسدان، كلمة موجهة المى المؤتمر الدولى لاتداد الداعين للدد من

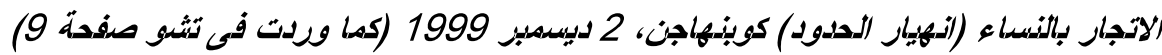

15 Article 3(a); see Stopping Forced Labour, A Global Report, the International Labour Conference, 89 Session 2001 Report I (B), International Labour Office, Geneva, ISBN 92-2-111948-3.

الدمادة 3. أنظل "وقف العمل الجبرى": تقريز دولى، مؤتم العمل الدولى، الجلسة 89 تقريي عام 2001 مكتب العمل الدولى، جينيف 
The UN High Commissioner for Human مفوض الأمم المتددة السامي لدقوق الإدسان

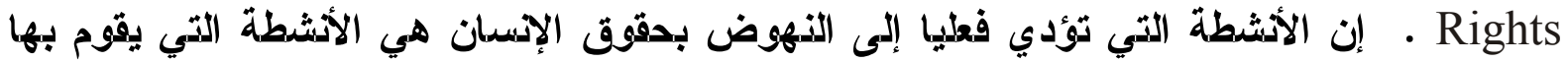

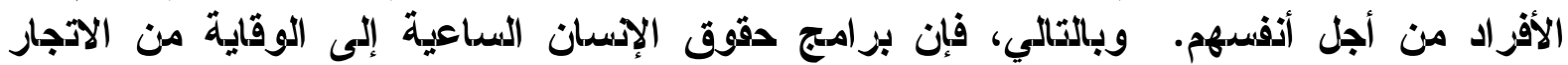

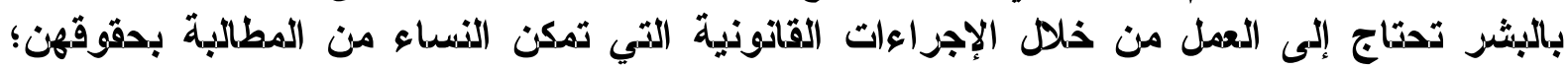

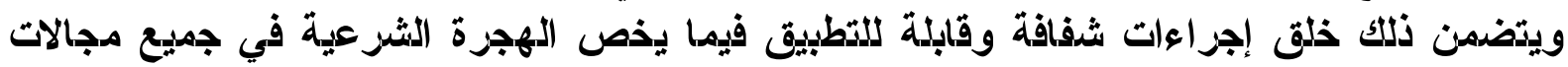

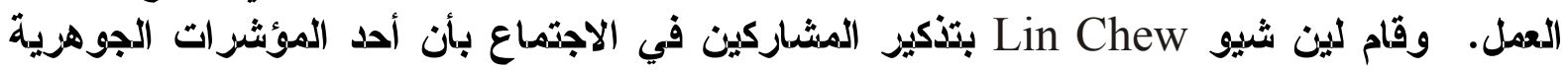

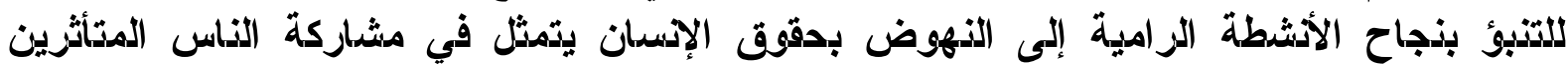

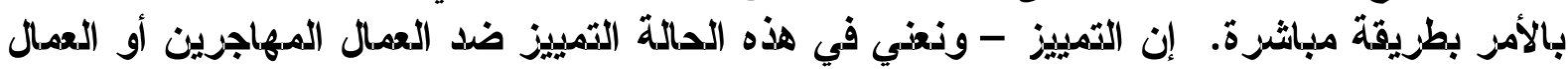

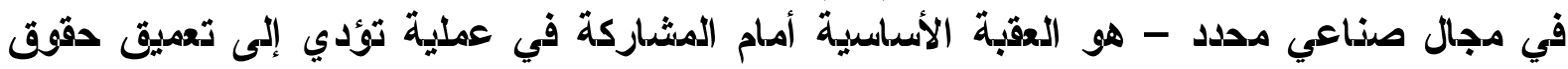

لا يمكن أن تكون الاستجابة القانونية للاتجار - سواء من خلا الآدفاقيات الدولية، أو القواعد التي

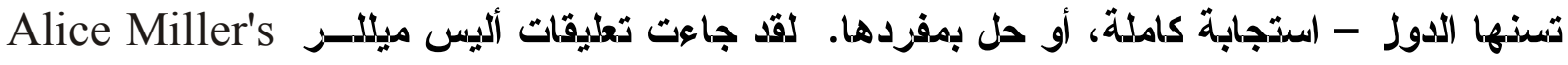

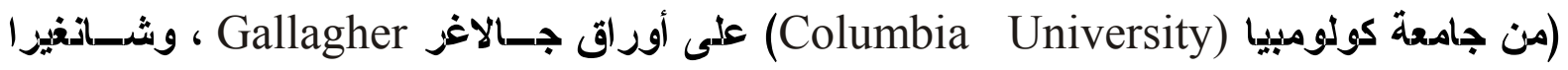
Sanghera

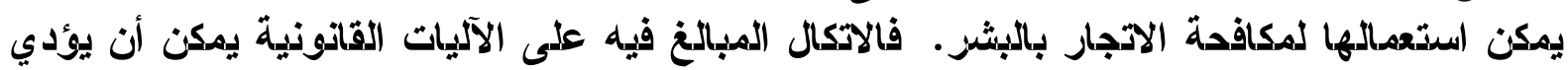

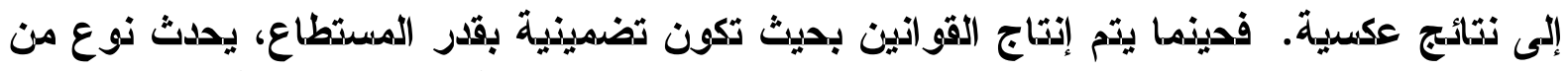

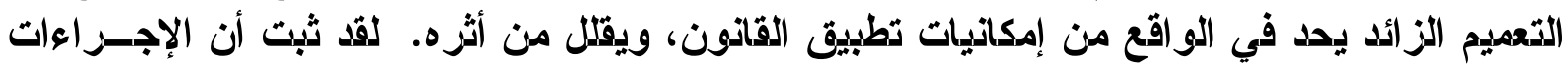

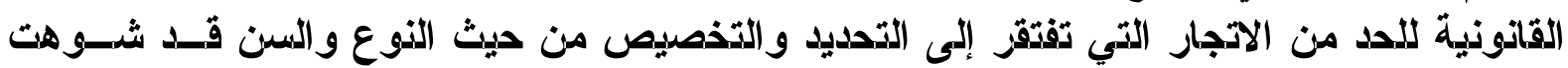

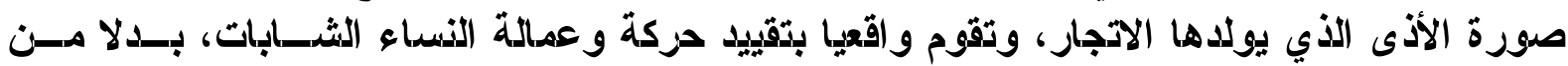

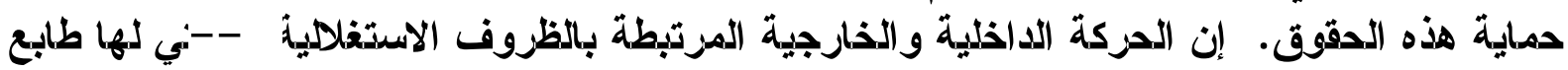

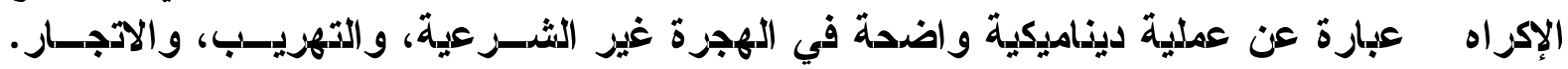

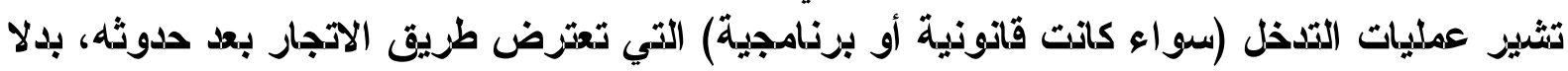

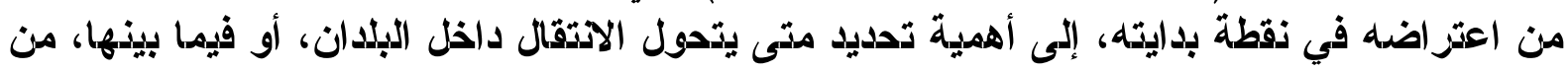

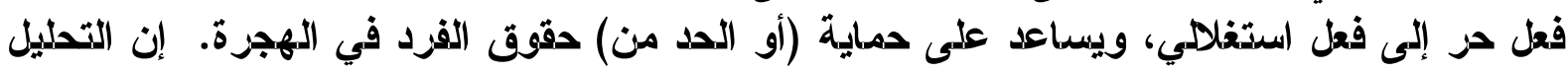

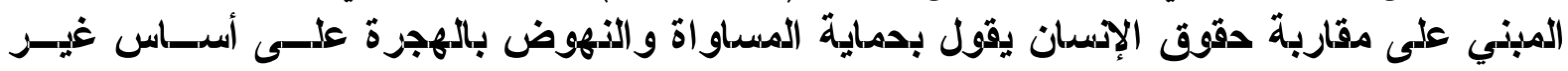
تمييزي.

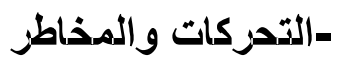
التضارب في التعبيفات الوظيفية للآجهار

قام بعد ذلك ماثيو فريدمان Matthew Friedman (هيئة المعونة الأمزيكية للتنمية الدولية/داكسا

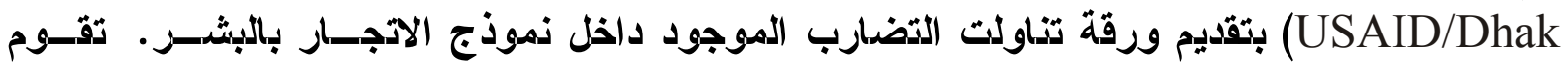

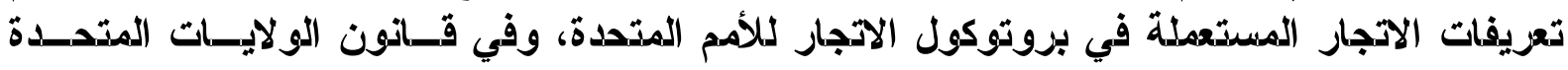
جوited States

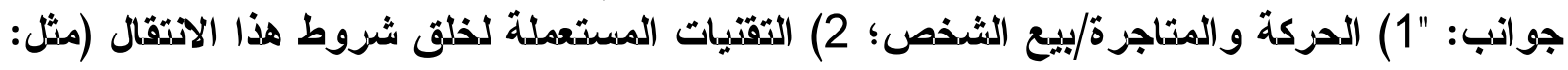

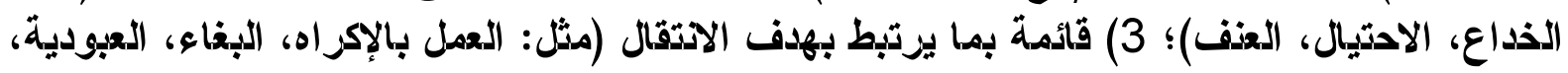




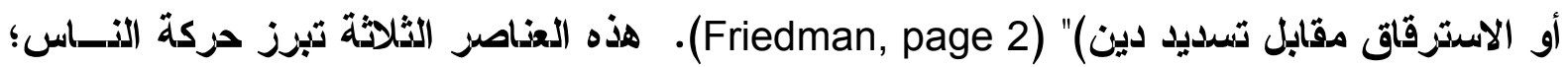

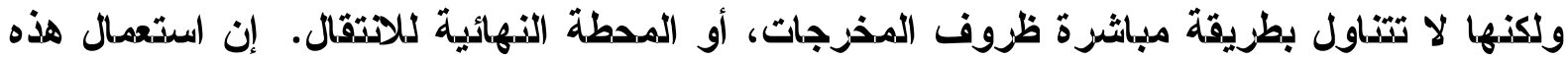

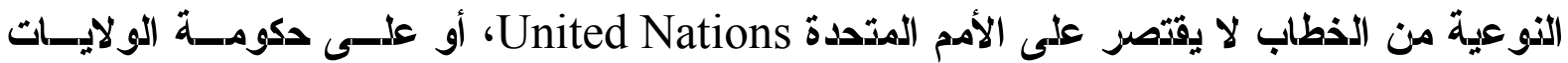

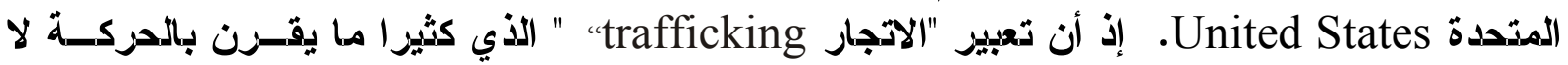

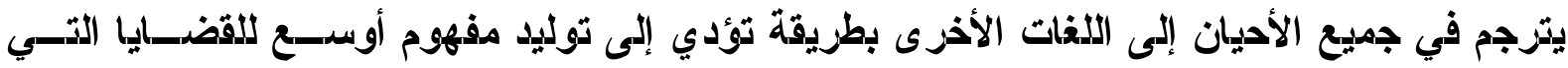

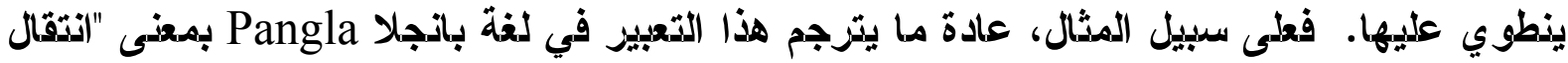

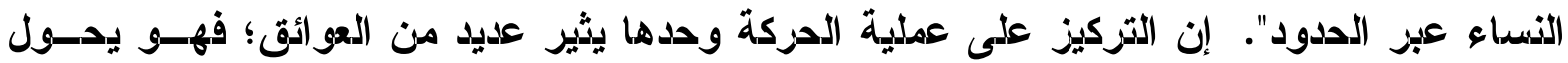

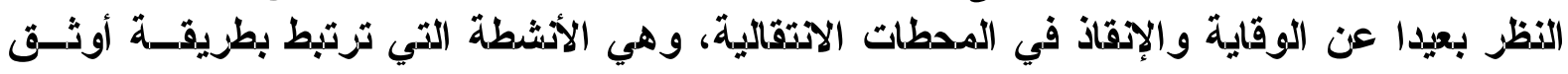

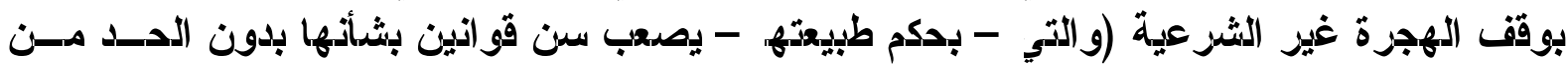
الهجزة)، بدلا من تحزي الآتهاكات في مكان (العمل.

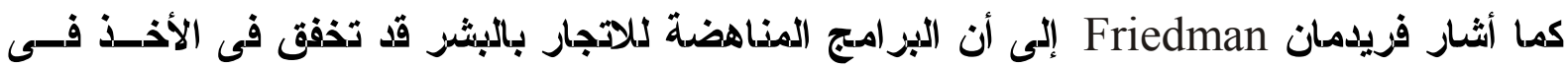

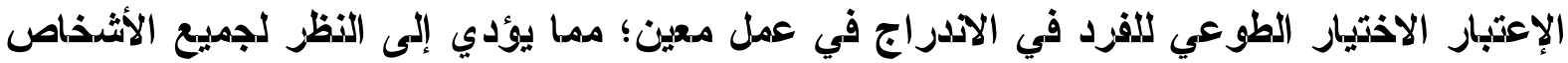

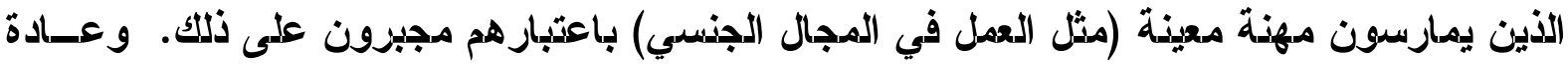

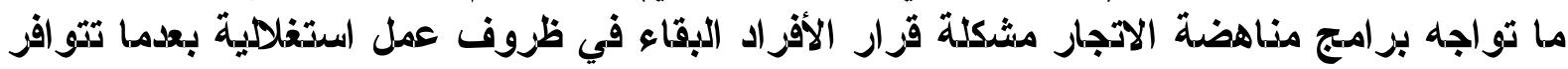

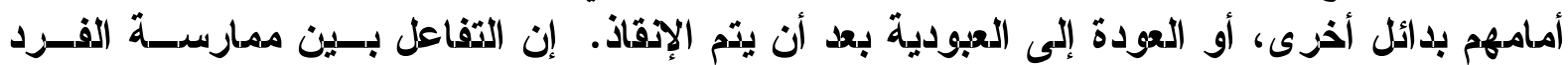

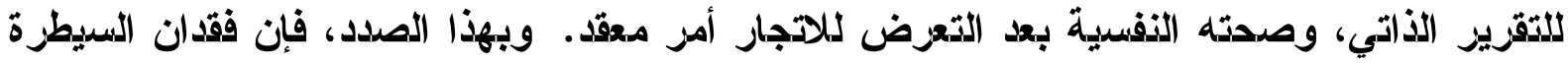

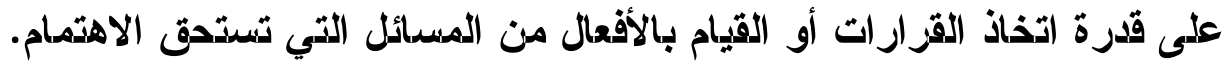

"..يعود عنصر أساسي لمشكلة الاتجار بالبشز إلى ما يلي: إذها عملية يفقد الشخص من خلالهما

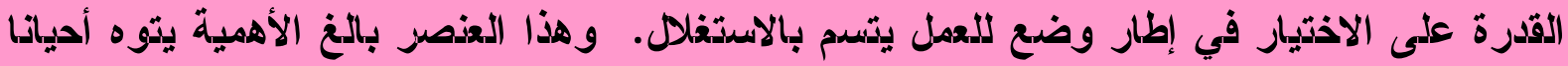

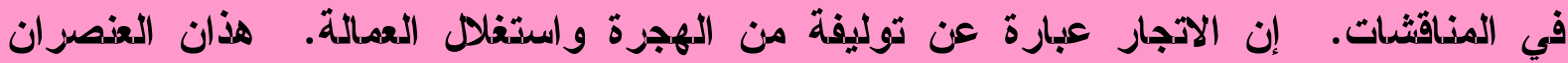

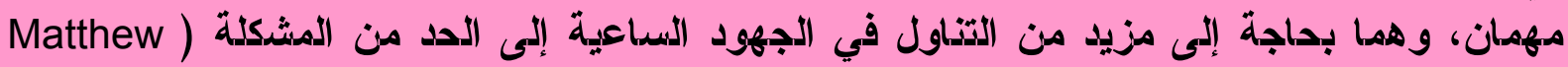
.(Friedman, page 5

Degrees of Vulnerability درجات المشاشة

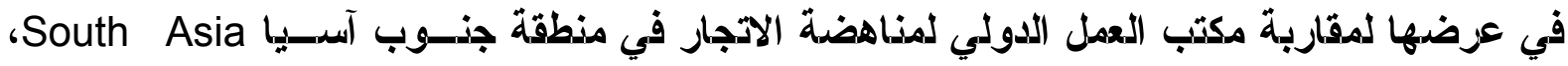

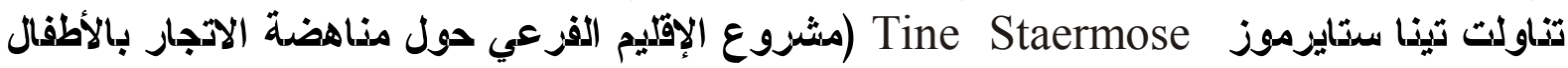
Sub-Regional Project on Anti-Trafficking in Childre

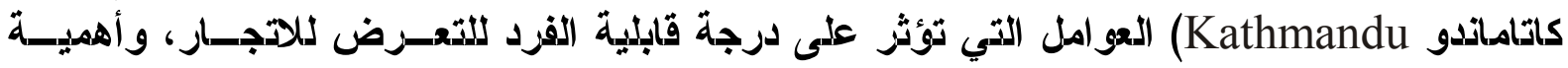

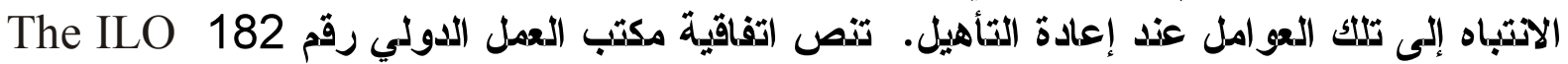
"جائ 182

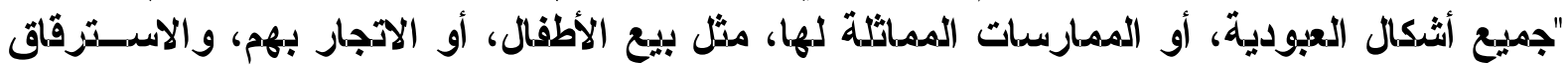

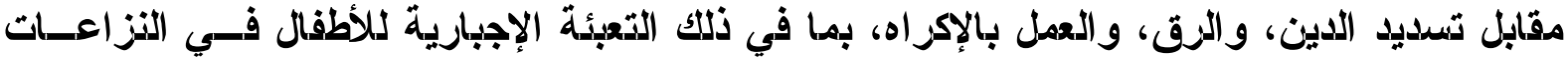




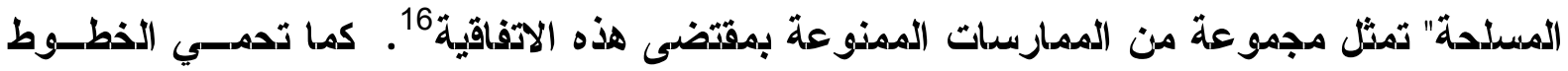

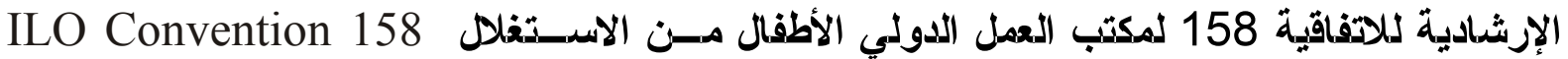
- guidelines

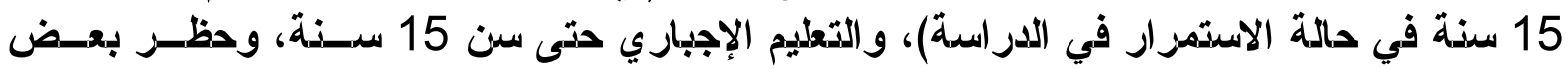
أثدكال العمل الذطيزة للأطفال حتى سن في الأن 17 سنة. والنعا

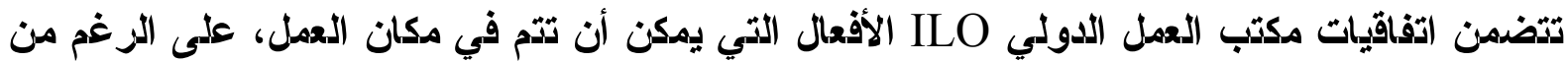

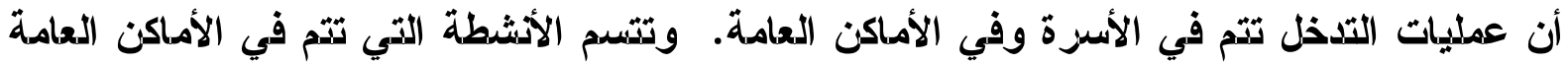

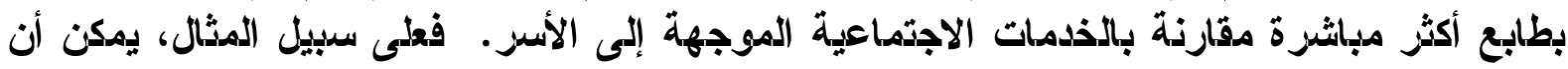

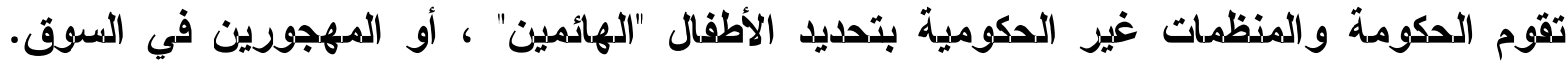

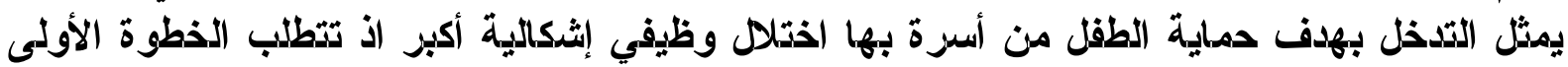

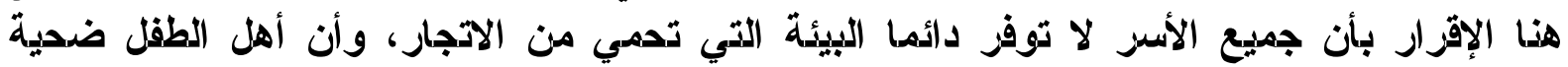

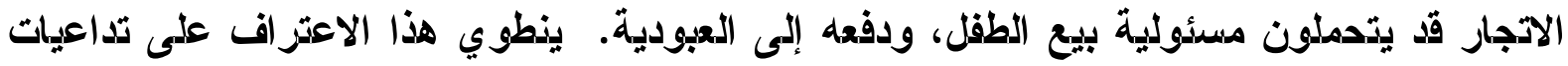

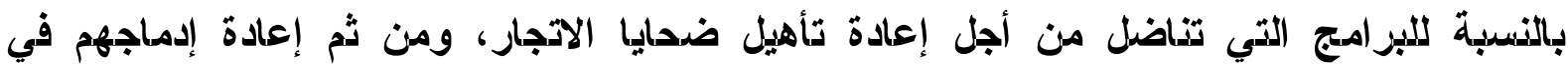
المجتمعات التي ينتمون إليها.

\section{Trafficking of Minors الاتجاربالقصر}

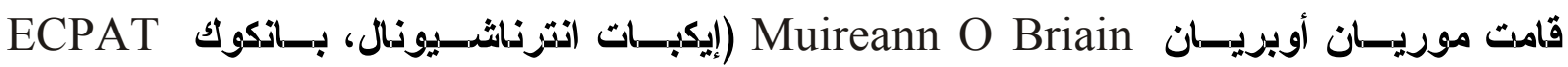
فائمارت (International, Bangkok

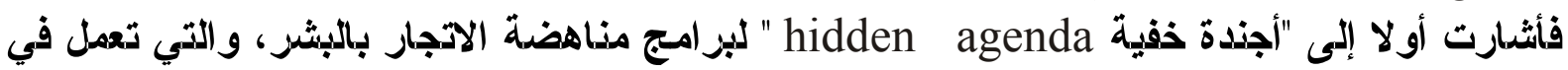

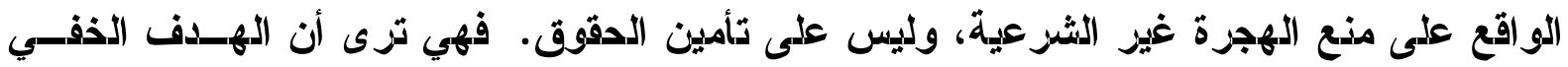

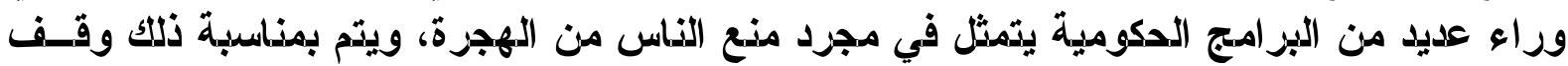

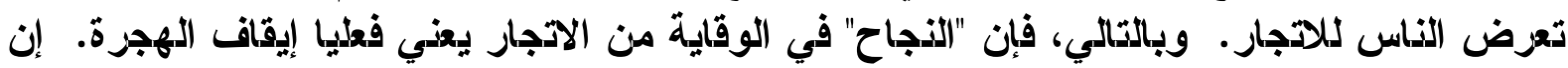

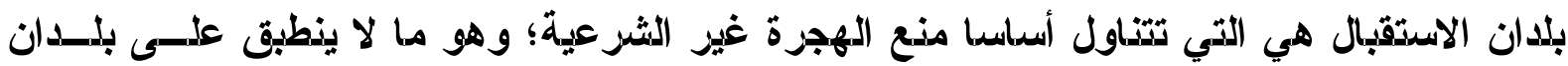

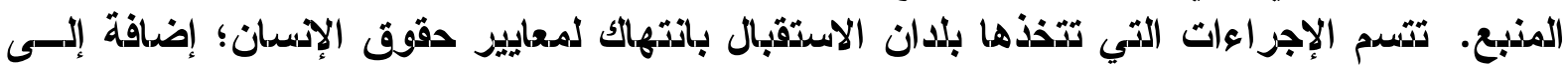

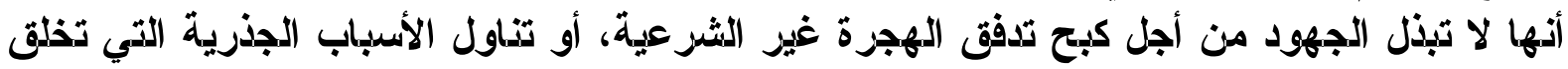

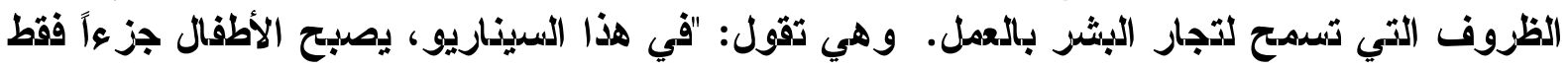

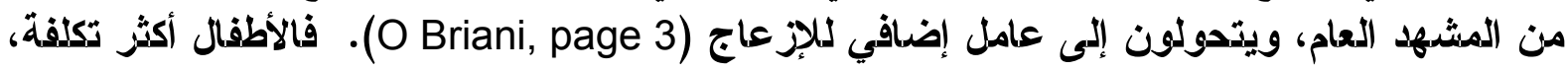

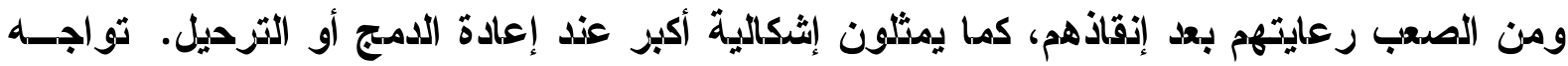

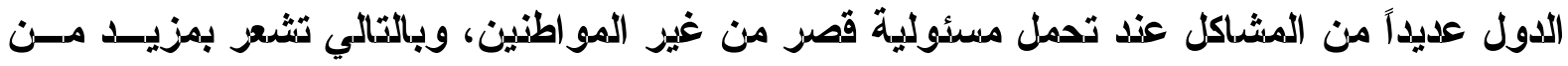

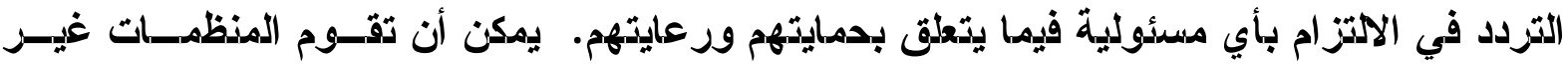

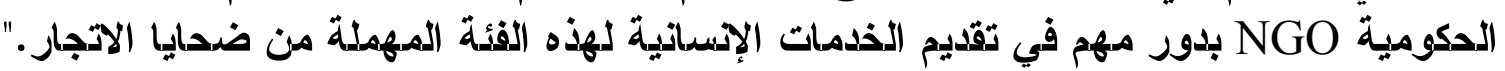

تعزف اتفاقية حقوق الطفل أي شخص تدت سن 18 سنة باعتباره طفلا تذطبق إجزاءات الدماية

${ }^{16}$ Source: Celine Costello Daly (presentation)

الدصدر: سيلين كوستيليو دالى (مد/خلة) 


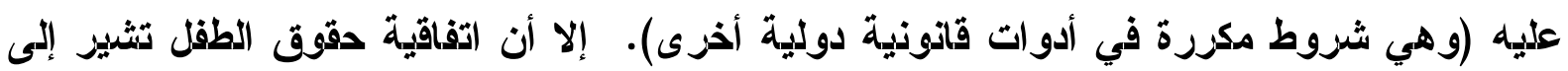

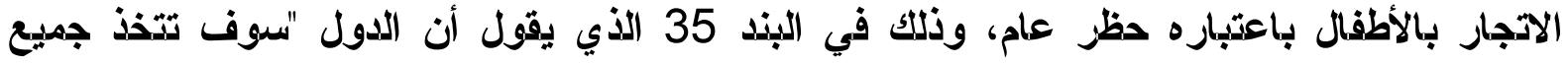

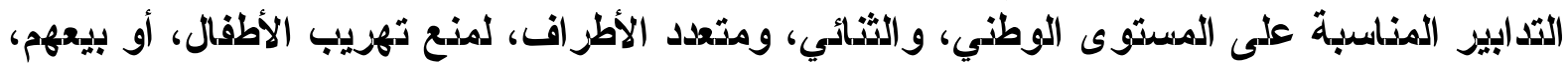

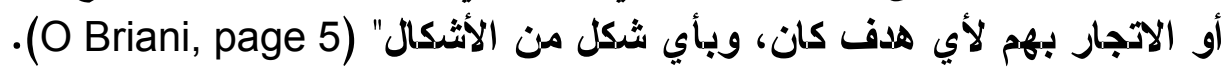

إن الاتجار بالأطفال عبارة عن فعل يقوم به المتاجز ضمن سلسلة الآتقال التي تعني التعبئة،

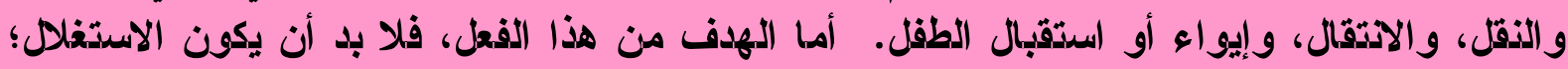

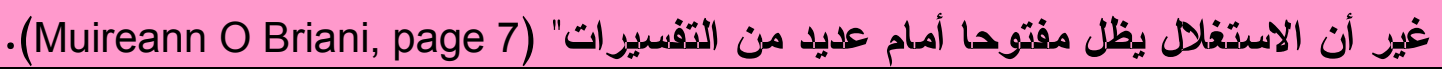

حيذما يتم ضم اتفاقية حقوق الطقل مع بروتوكول الاتجار بالبشز، والآتفاقية 182 لمكتـب العهـل

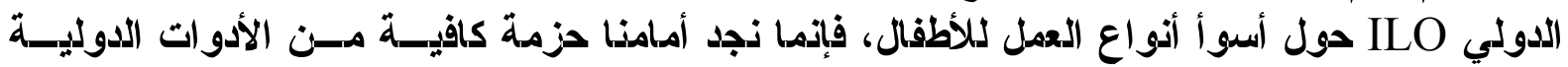

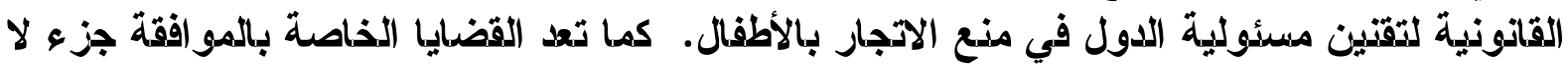

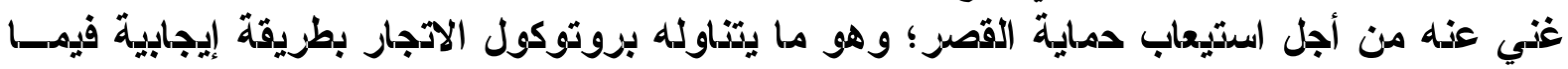

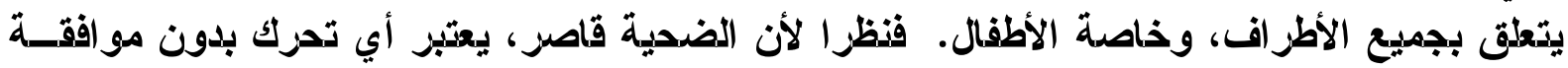

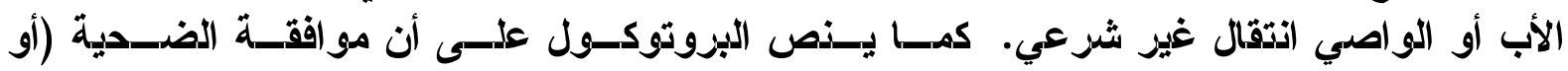
th he الأهل/الوصي الوصي parent/guardian

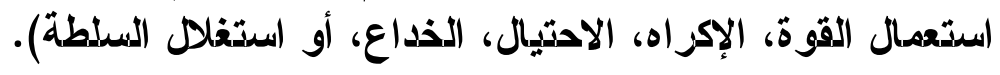

إن الأذى الممتد الذي يصيب الأطفال ضدايا الآجار مصدر قلث للمجتمع. فيتم الاتجار بالأطفال

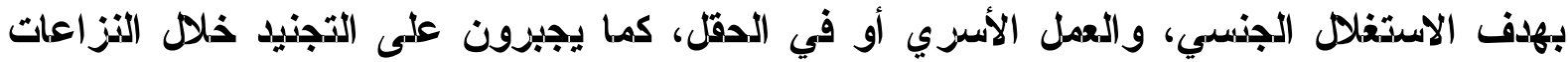

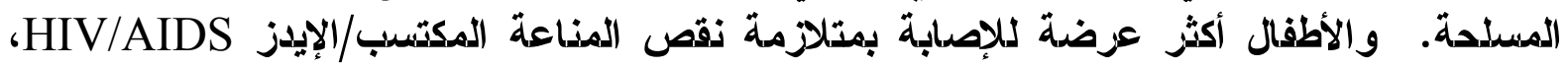

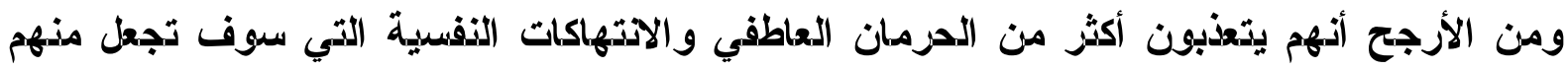

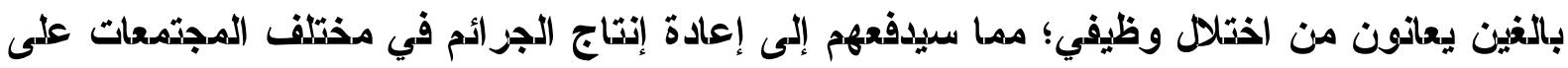

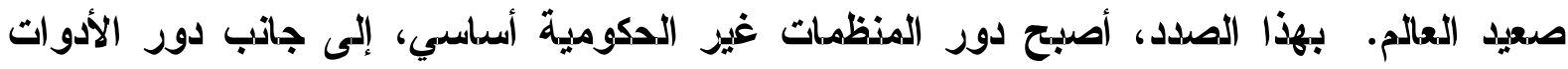

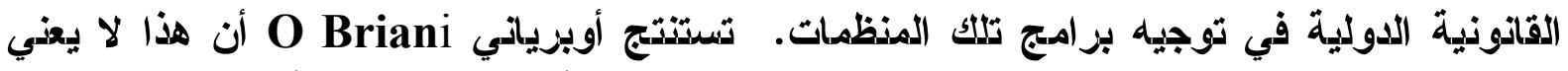

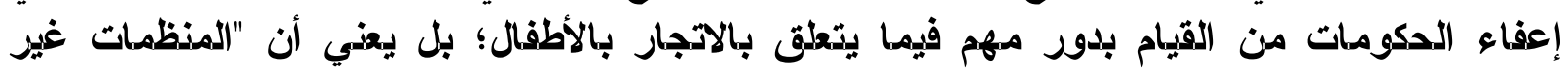

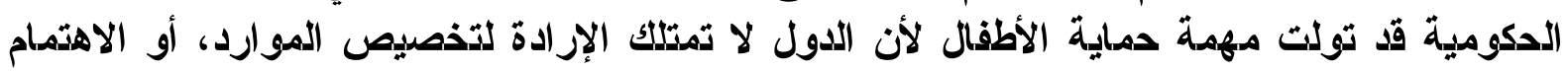

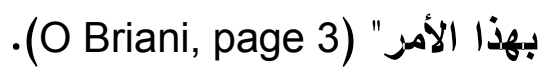

قام فيل مارشالPhil Marshall (مشروع الأمم المتحدة فيما بين الهيئات حول الاتجسار بالذسـاء

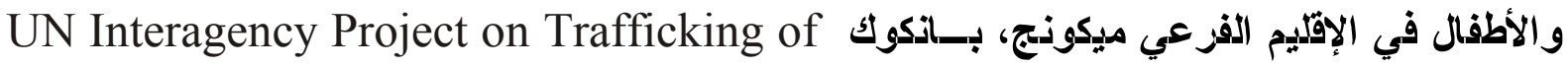

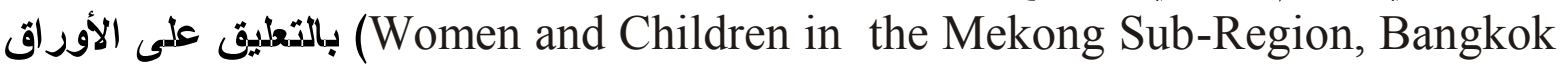

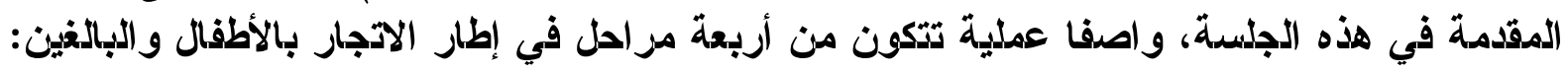

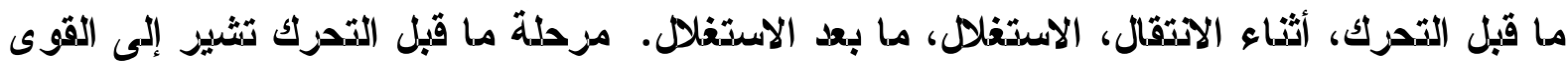

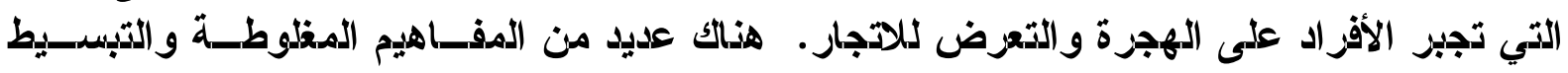

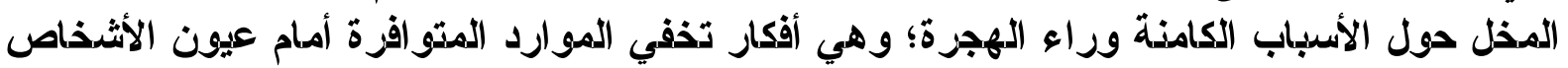




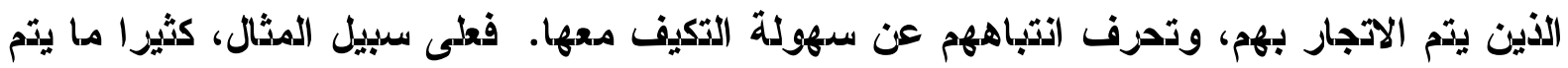

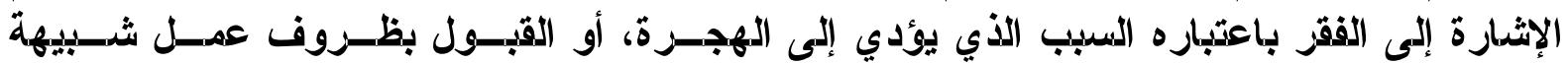

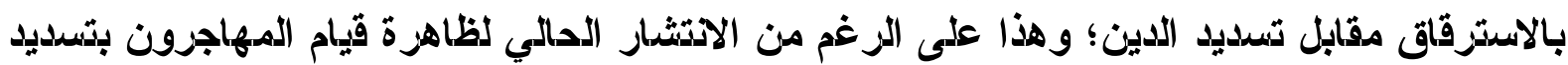

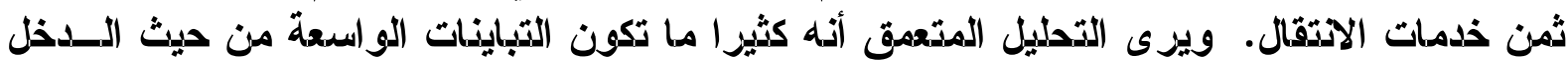

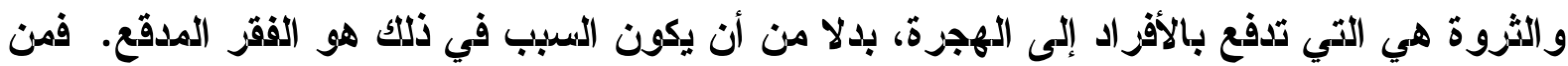

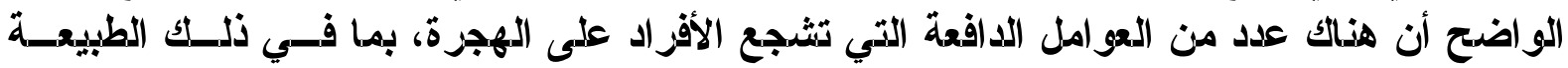

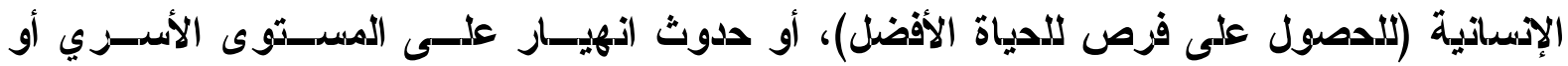
الاجتماعي، إلى جانب السبب الذي فيتم الإثشارة إليه باستمرار، ألي الذئ الفقر.

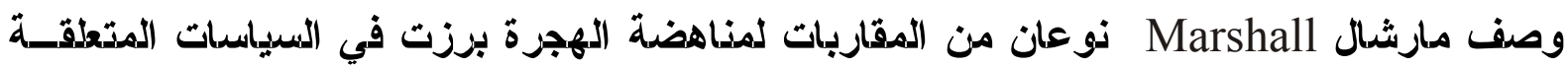

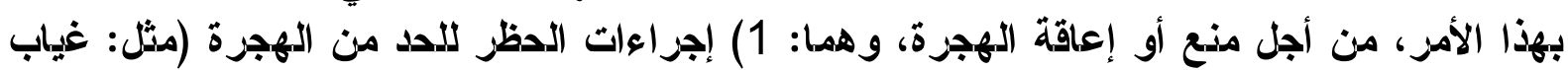

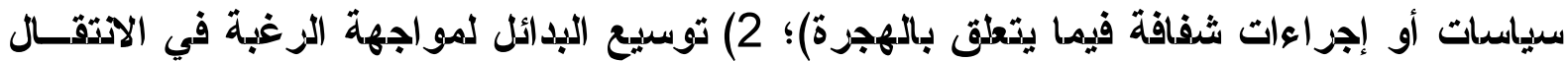

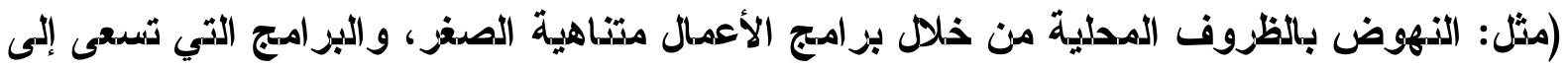

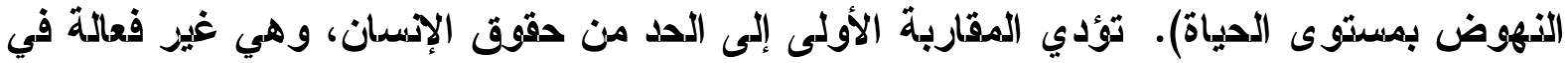

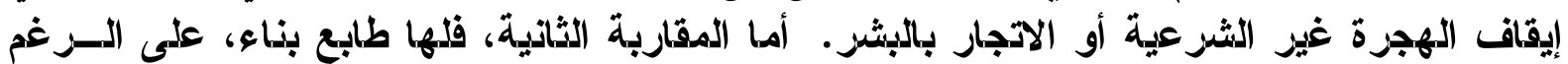

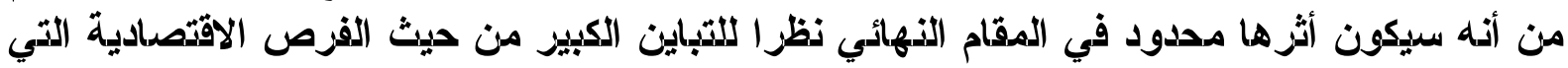

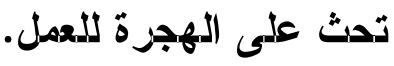

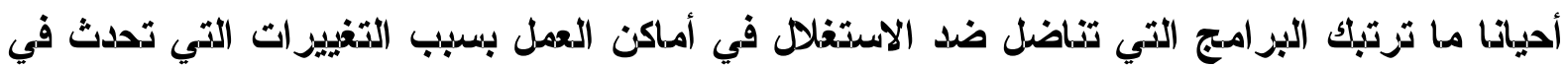

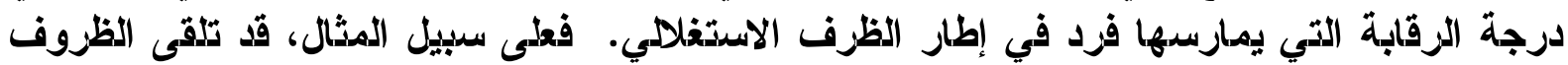

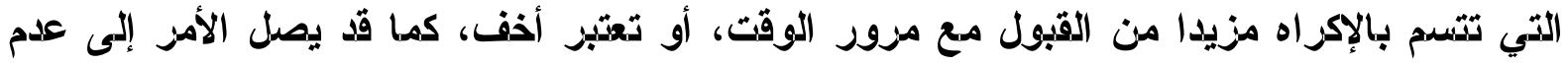

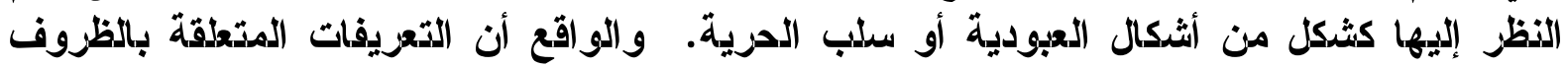

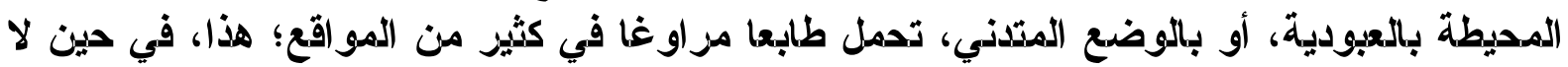

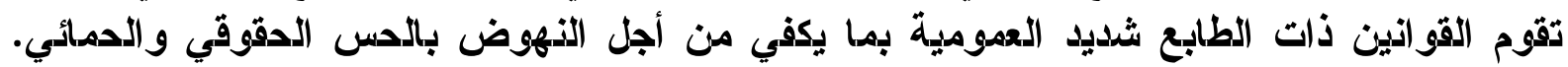

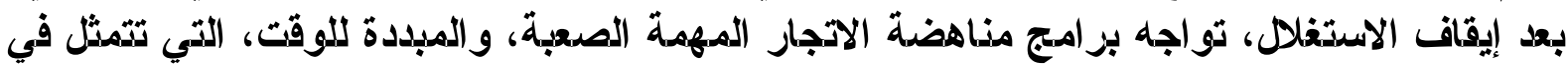

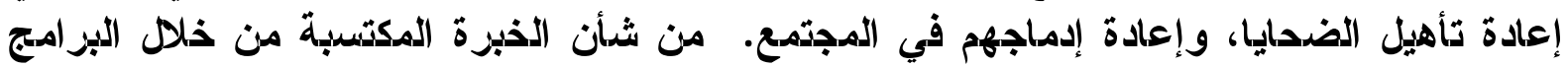

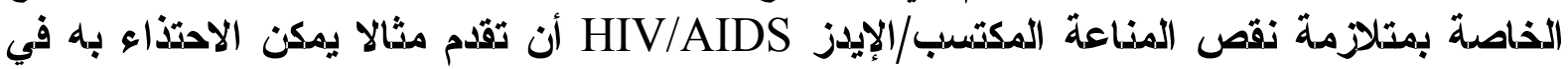

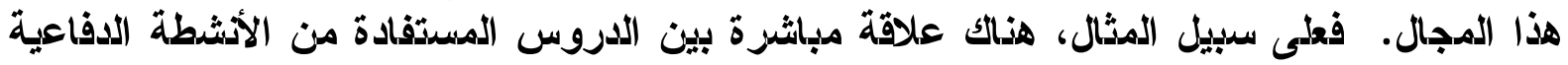

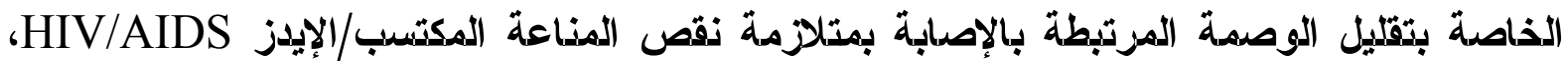

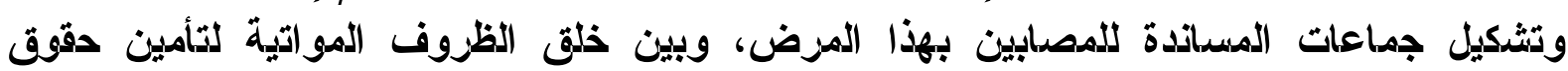

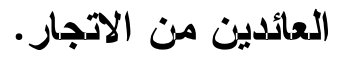

IDENTI FYING INTERVENTION MODE L S تحديد نماذج للتدخل - الأوقاية، الزعاية، الدعم، وإعادة الإدماج 
IOM'S Anti-Trafficking المقارية المكتب الدولهي للهجرة في مناهضة الاتجار بالبشر Approach

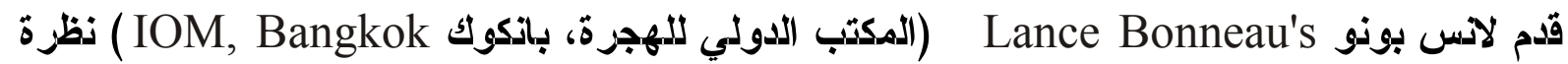

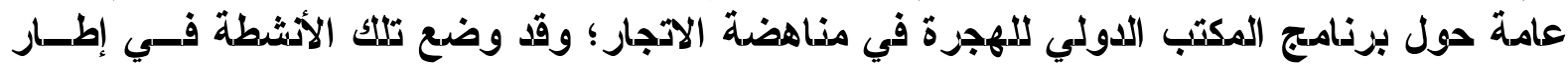

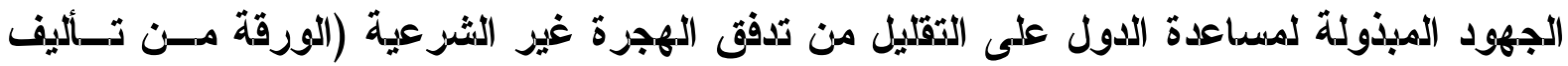

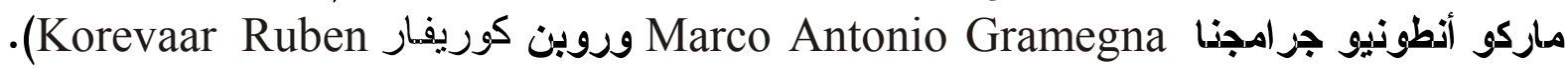

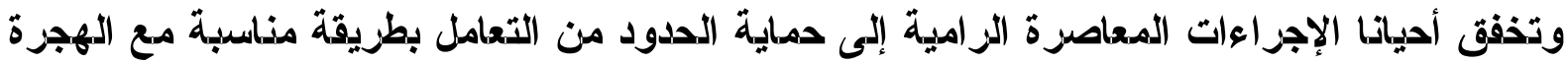

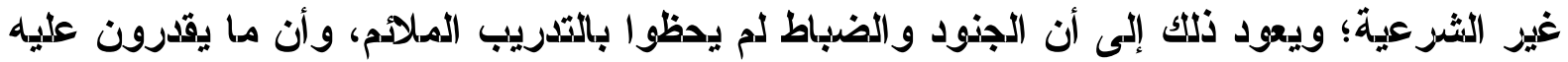

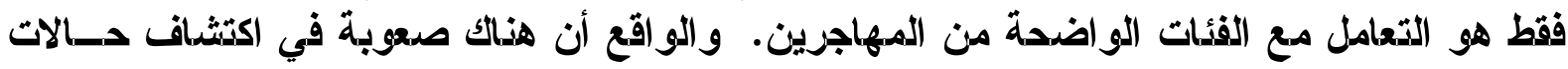

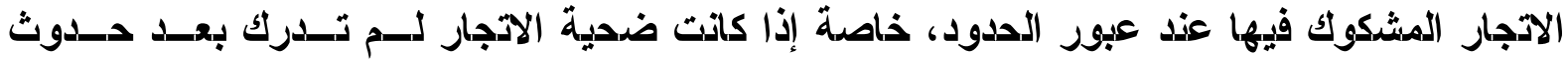

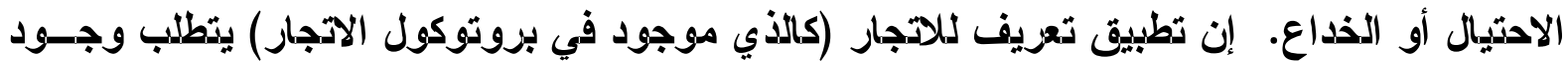

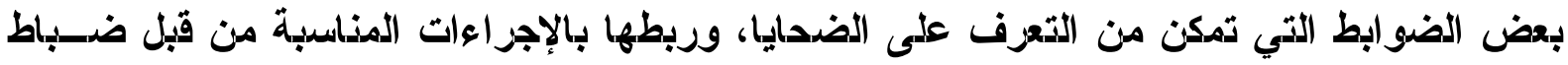

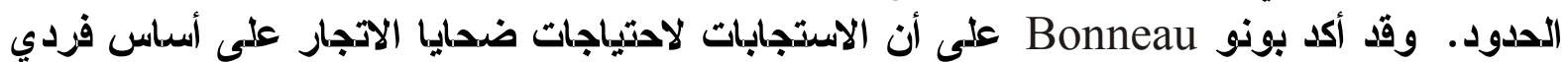

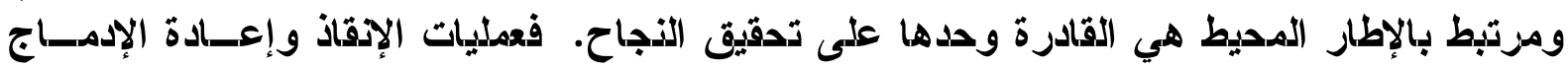

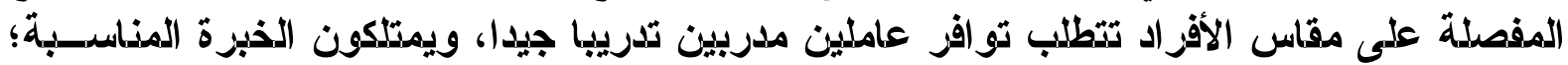

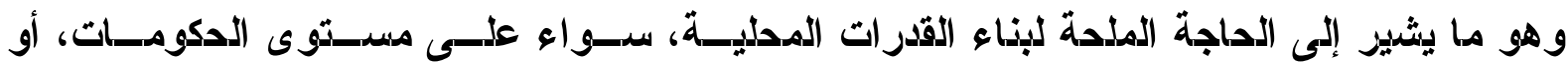

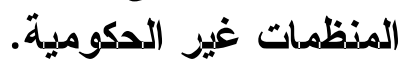

"هناك اليوم اتفاق عام فيما بين الدكومات والهيئات الدولية على أن الاستجابة الوحيدة القابلة

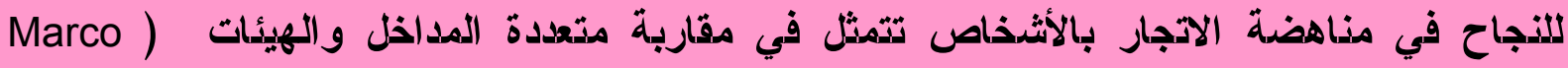
.(Antonio Gramegna and Ruben Korevaar, page 1

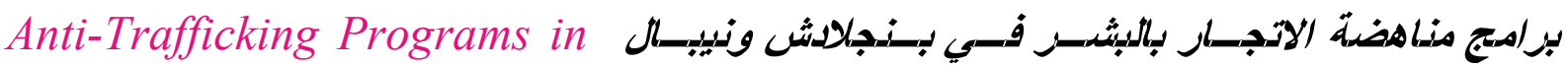
Bangladesh and Nepal

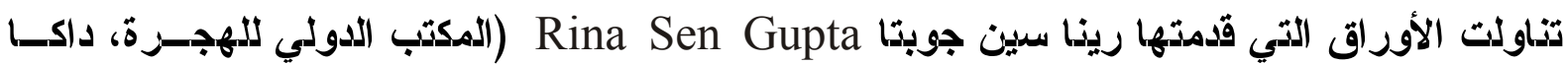

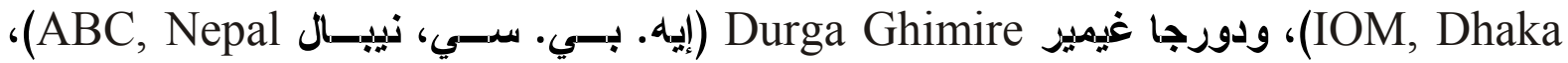

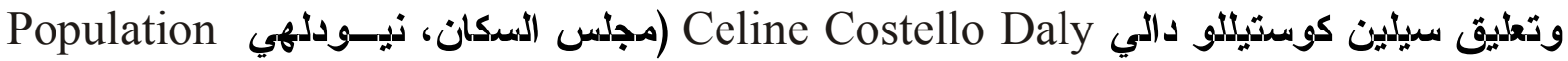
(Council, New Delhi

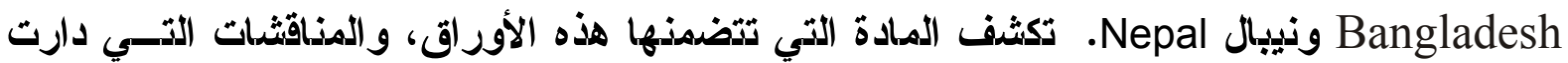

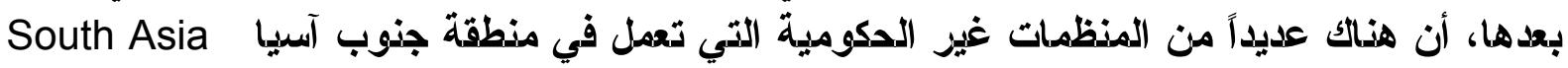

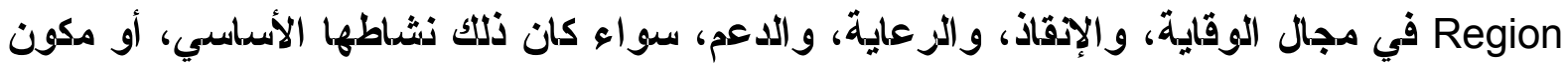

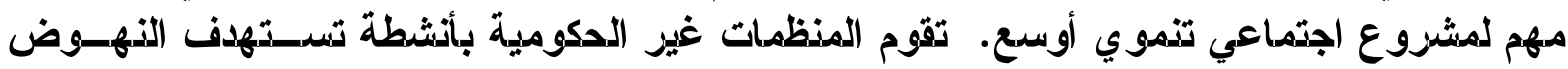

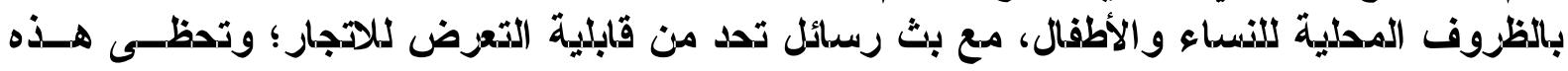

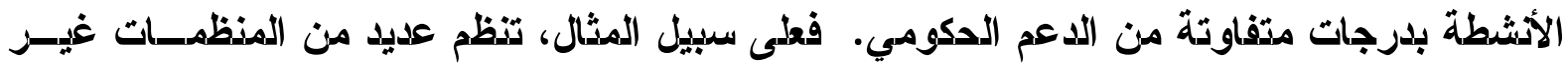


الدكومية في نيبال Nepal و والهند India، وينجلادش Bangladesh دورات تدريبية حول مهارات

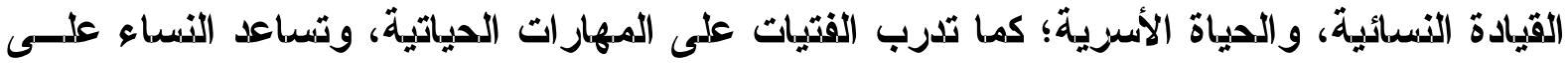

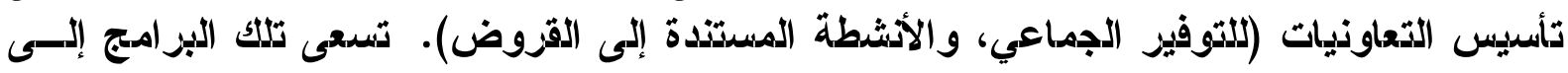
تحسين الظروف التي كثيرا ما اعثبرت السبب والأبطامن الكائ وراء الاتجار بالبشز.

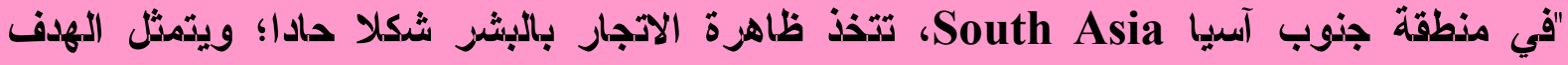

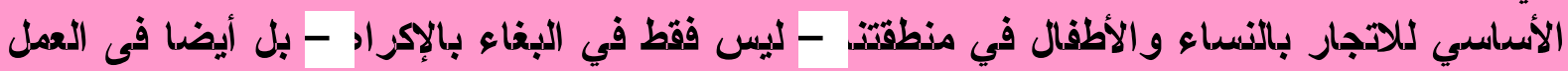

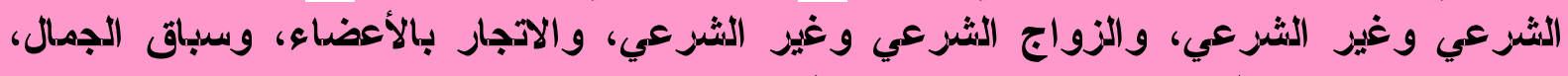

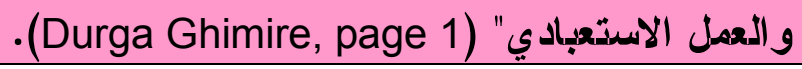

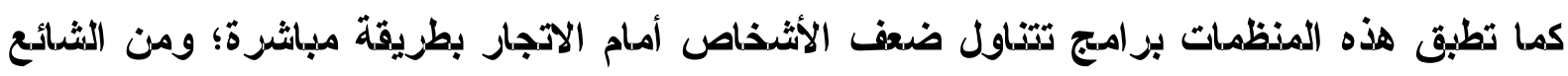

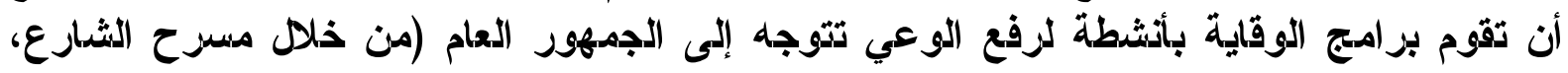

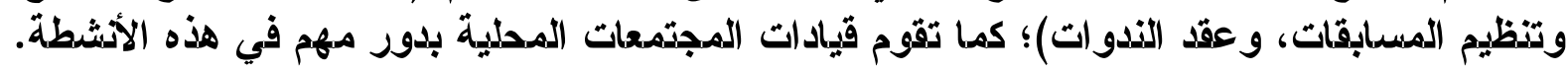

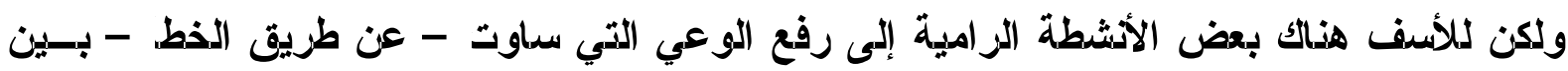

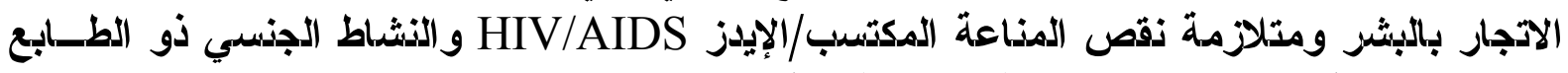

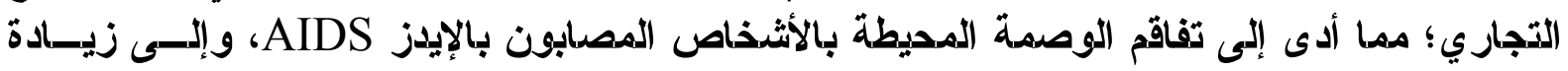

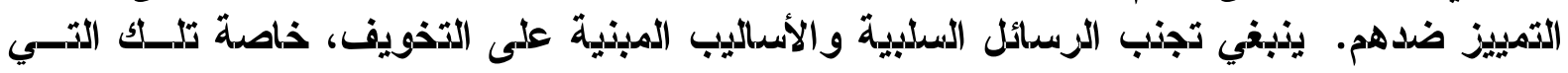

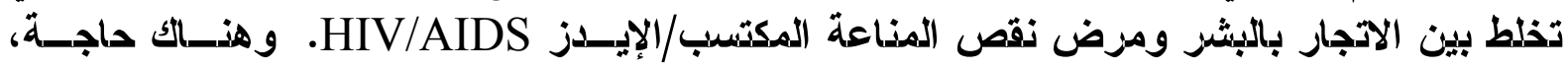

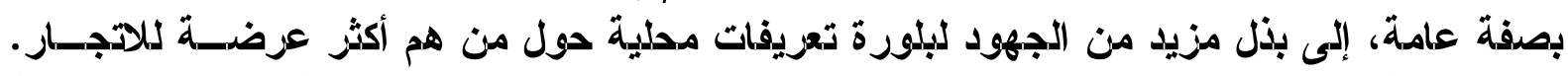

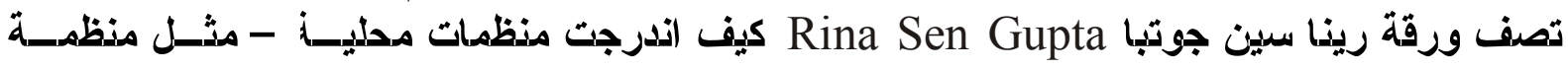

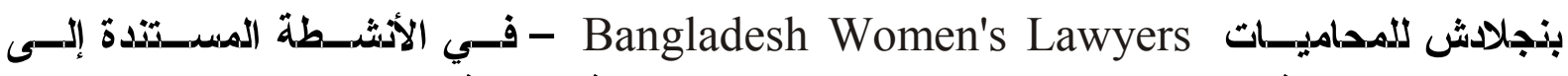

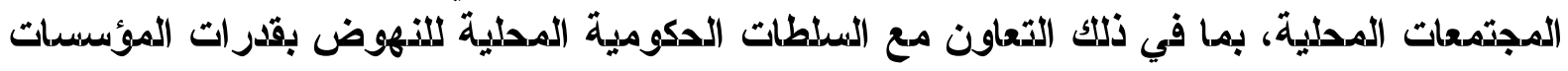

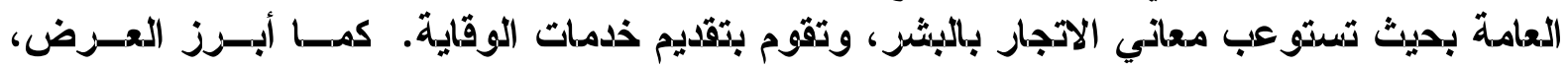

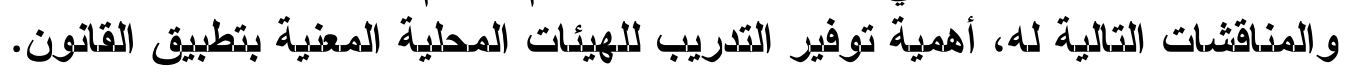


عمليات التذخل التي تتجه إلى المزاحل المذتلفة للاتجار بالبشر

المجتمع المدلي أو مكان العمل

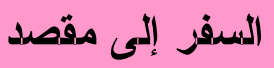

فتزة من العمل غير التطوعي مفيد

إعادة التأهيل

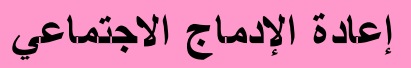

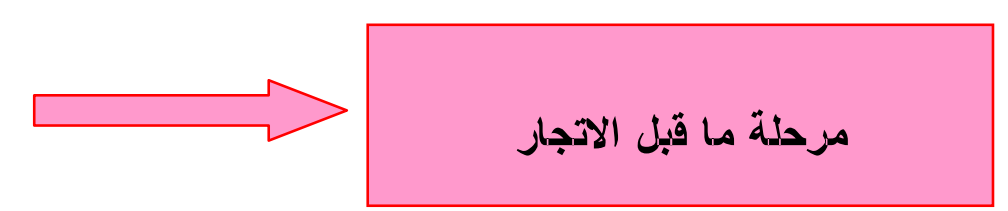

مزحلة ما قبل الاتجار
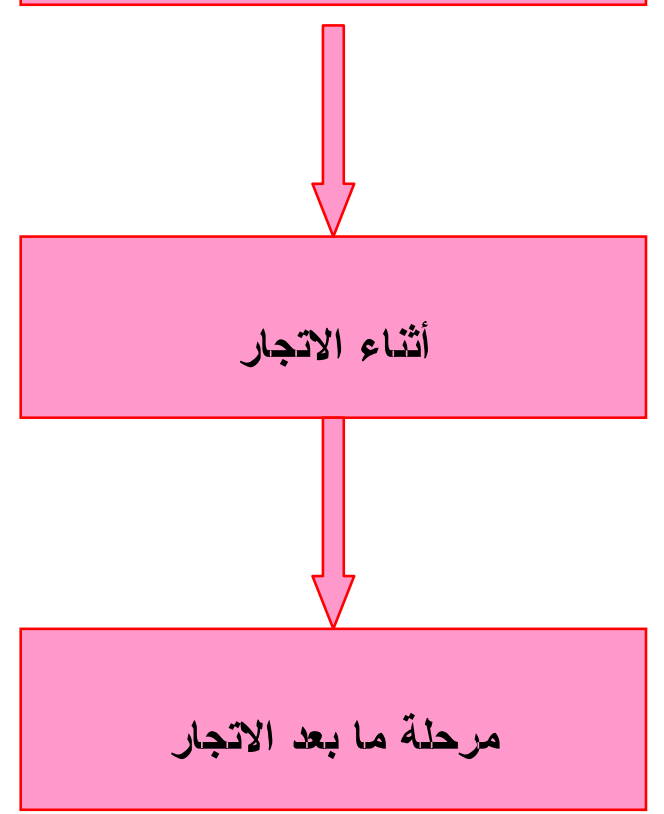

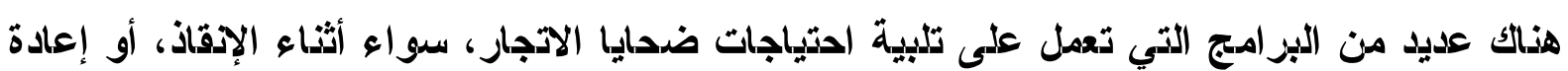

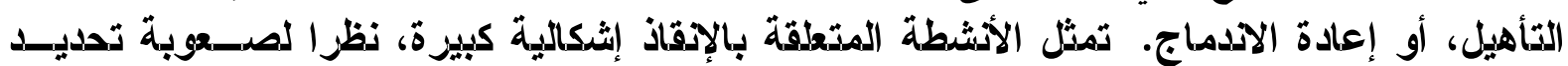

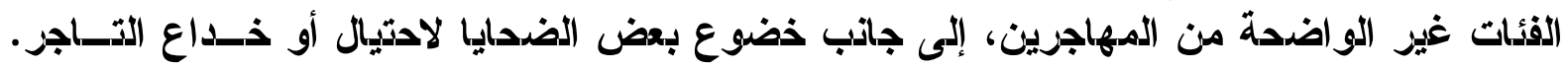

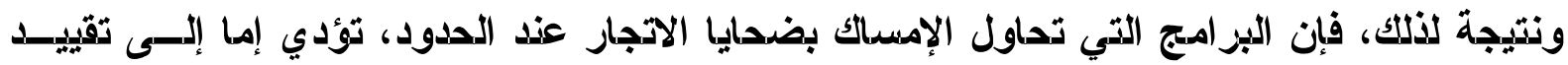

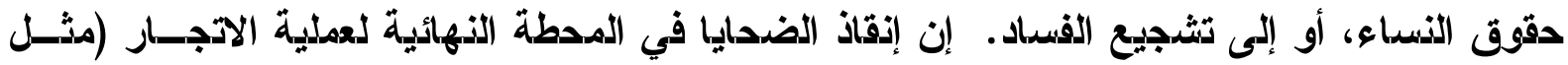

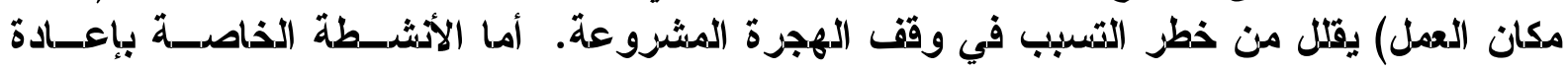

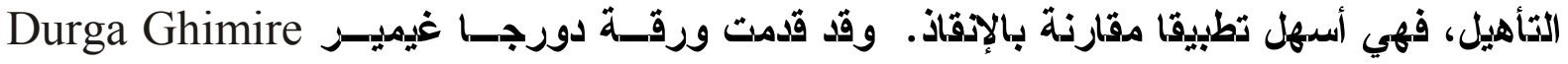

17 The South Asian Association for Regional Cooperation draft "Convention on Preventing and Combating Trafficking in Women and Children, SAARC/SUMMIT 10/CM.20.3, cited in Ratna Kapur, page 4.

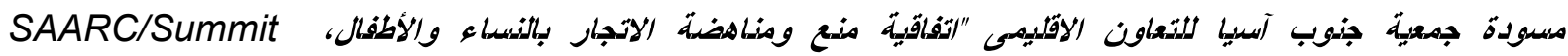
10/CM20.3 كما وردت في راتنا كابور صفحة 4 
خصائص برامج إعادة التأهيل الناجدة في الدد من العزلة، وتجنب الظروف التي تولــد الوصدمة،

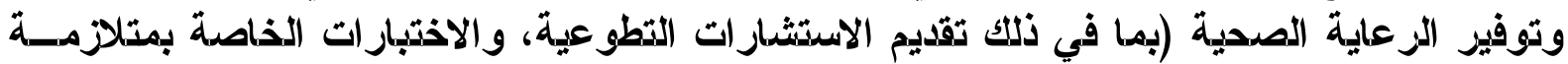

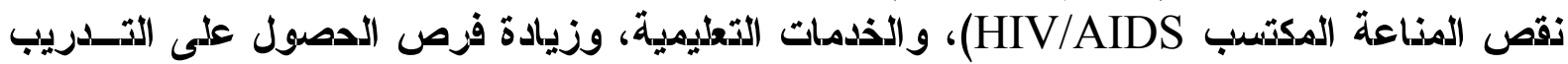

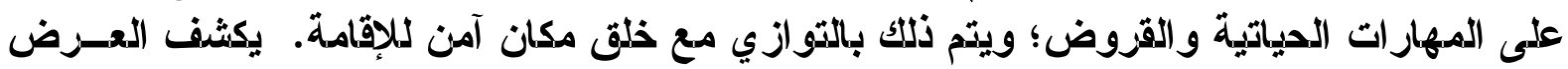

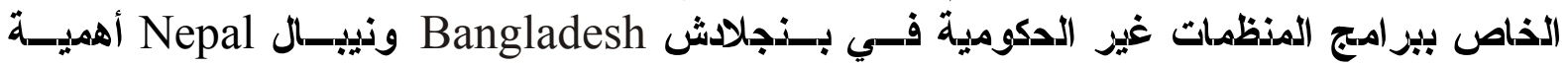

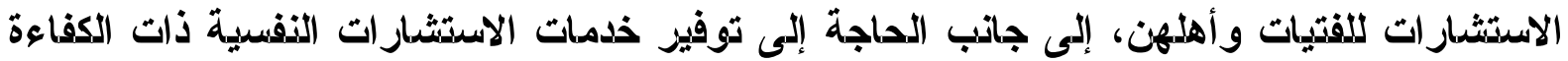

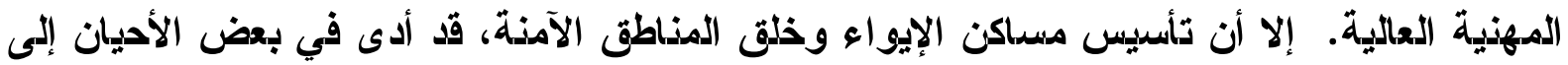
عزل ضدايا الاتجار، وسماهم في توليد الوصمة الونة، بدلا من تقليلها.

من الممكن أن تتعقد عملية إخراج شذص تم الاتجار به من مرحلة إعادة التأهيل إلى مرحلة إعادة

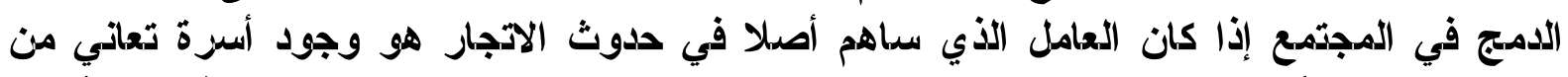

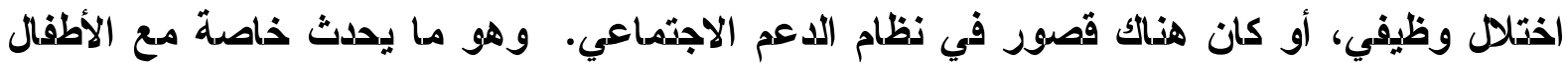

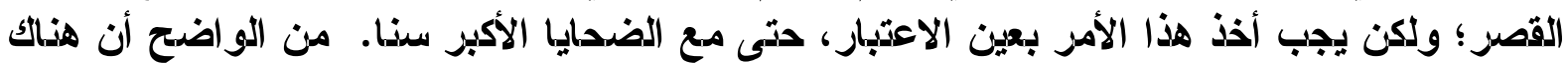

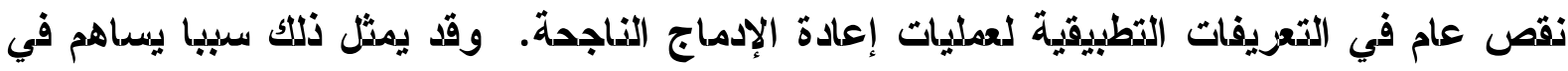

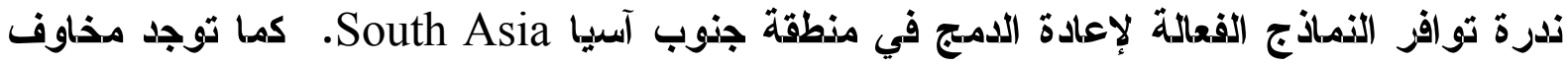

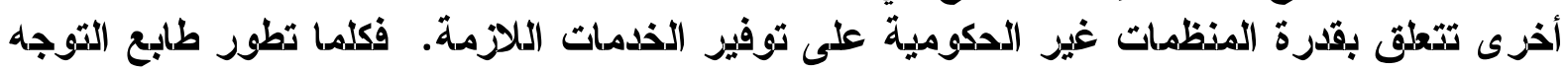

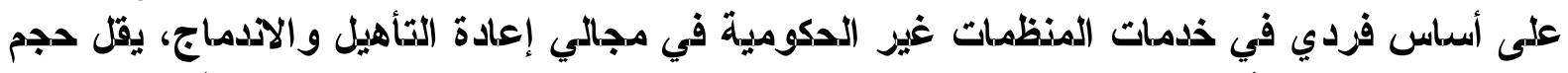

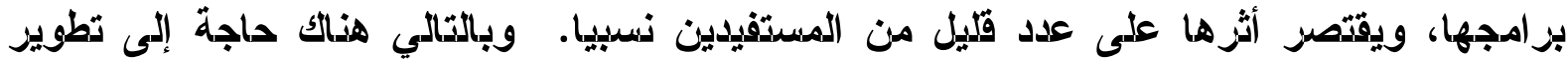

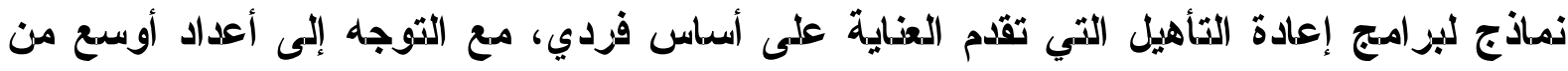

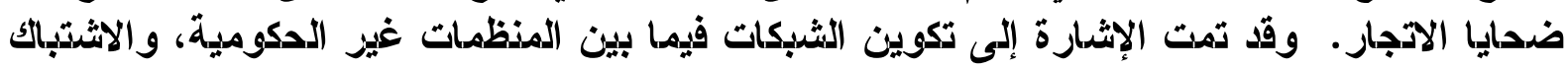

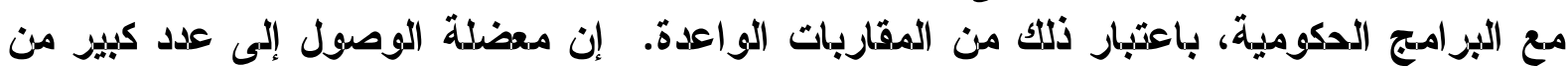

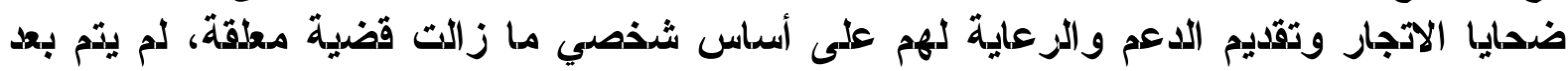

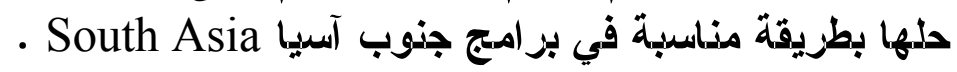

Law Reform, Advocacy and الإصلاحات القانونية، والأنشـطة الدفاعيسة، و المقاضـاة

Prosecution

Obstacles to Successful Prosecution العقبات التي تعتزض النجاح في مقاضاة تجار البشر

of Traffickers

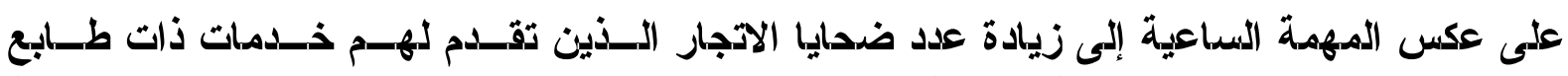

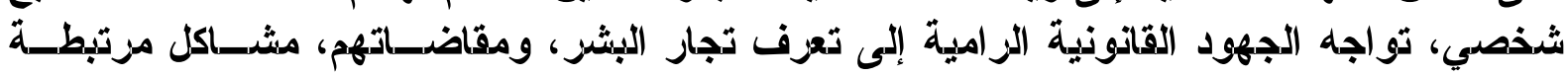

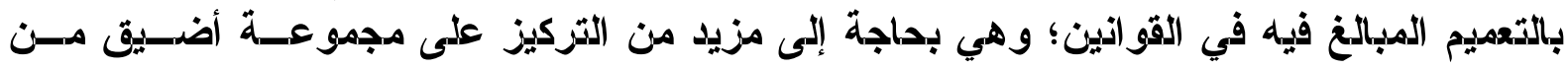

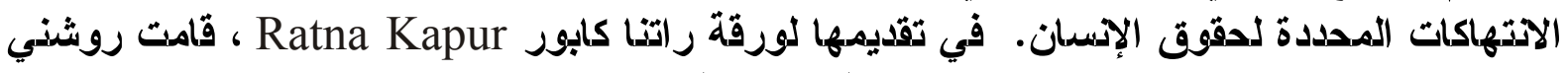

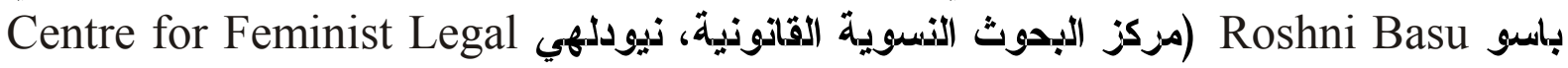
Research, New Delh ) بتوصيف مدورين واسعين يعوقان تطويز الإصلاحات القانونية الفعالة في مذطقة جنسوب آسـيا،

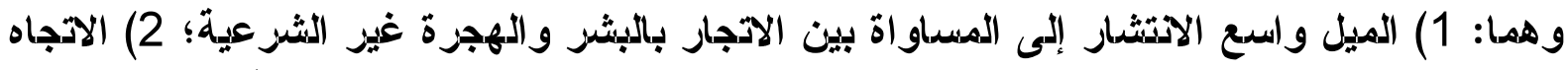

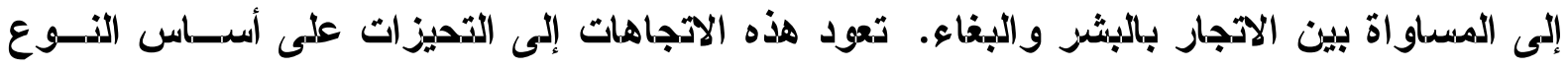




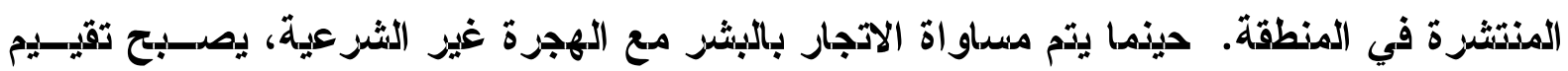

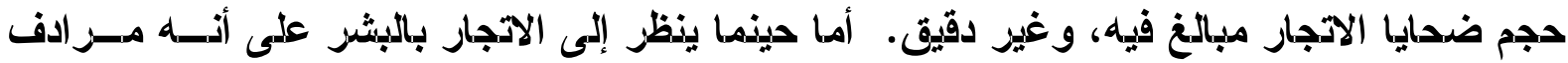

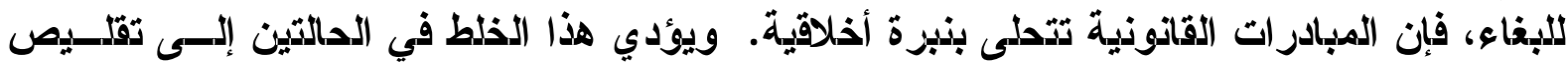

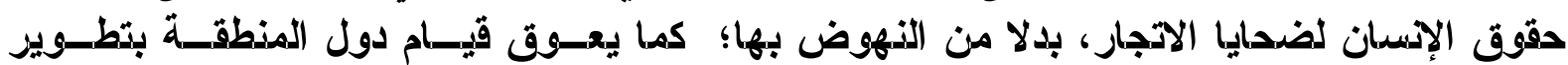
الاستجابات القانونية المناسبة.

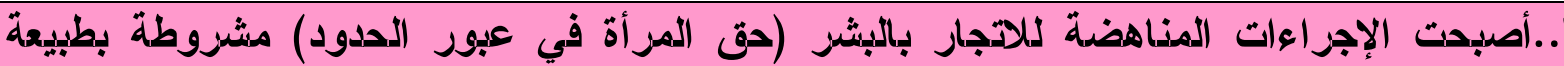

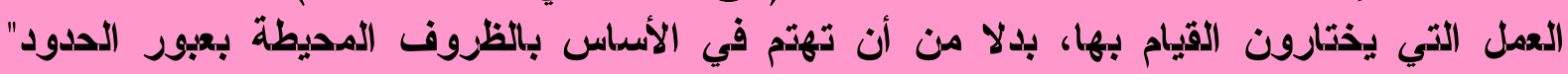
.(Ratna Kapur, page 11)

كما تؤثز التحيزات المبنية على أساس الذوع على طريقة تفسيز القوانين، وإجزاء المحاكمات، بمسا

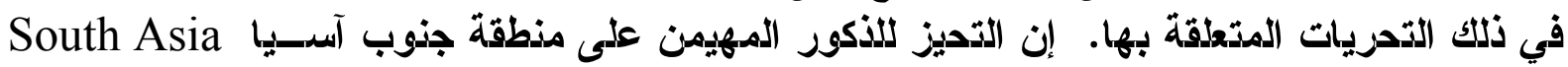
Region

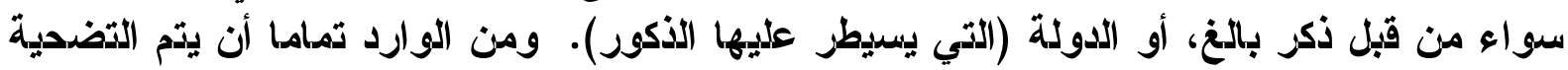

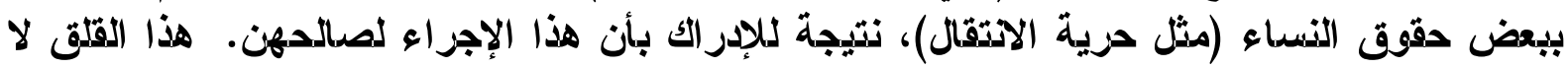

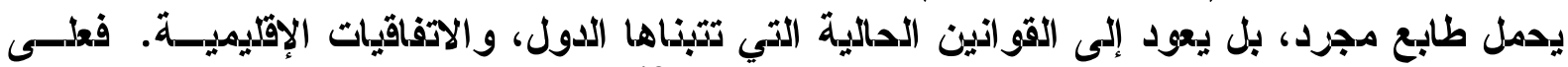

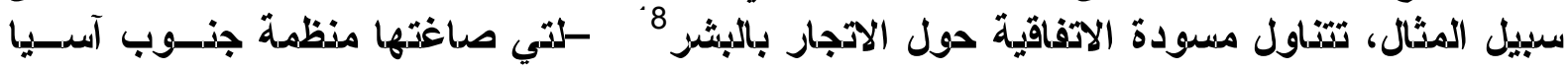

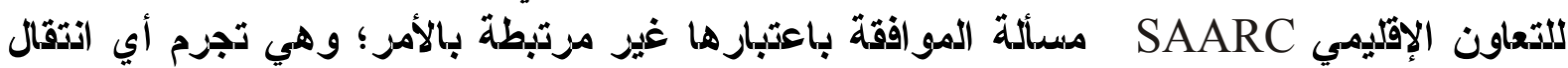

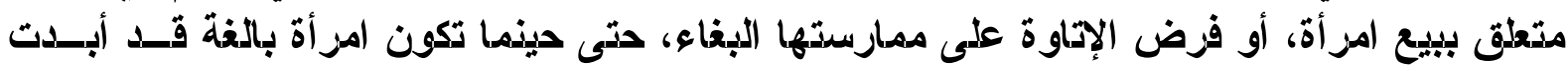

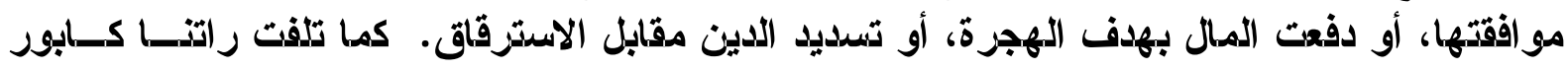
Ratna Kapur

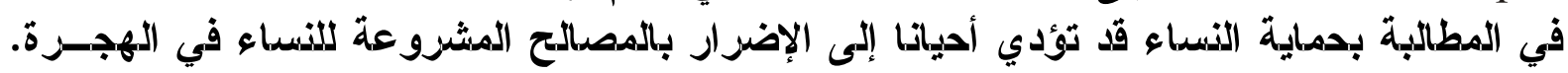

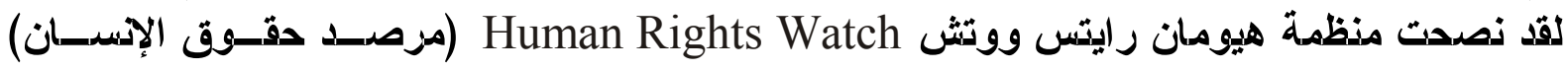

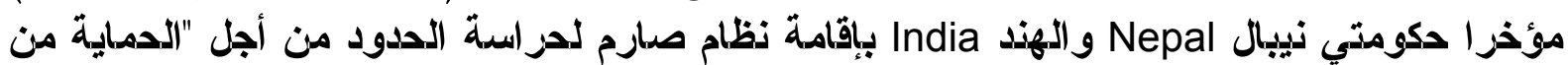
الاتجار بالنساء والفتيات"

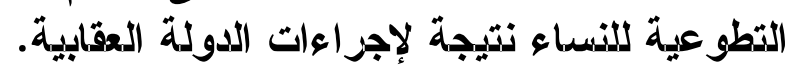

بدون تجاهل هذه المخاوف، أثثارت ناتديتا بارو Nandita Barauh (صندوق الأمم المتحدة لإدماء

18 The Human Rights Watch Global Report on Women's Humans Rights (1995), cited in Ratna. Kapur, page 9.

الثقزييز الدولى لزصد حقىق الاسان حول حقق الاسان الذاصة بالنساء (1995) كما وردت فى راتنا كابور صفحة 9

${ }^{19}$ Handbook of Indicators for Family Planning ProgramEvaluation, Ian Bertrand, Robert Magnani and James Knowks, The Evaluation Project, Carolina Population Center, University of North Carolina of Chapel Hill, 1994

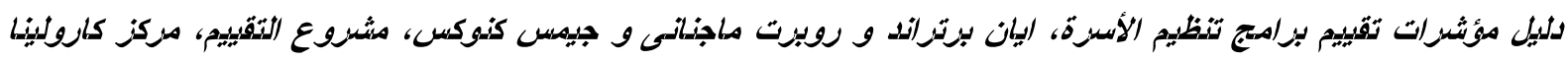

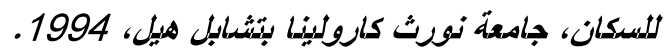




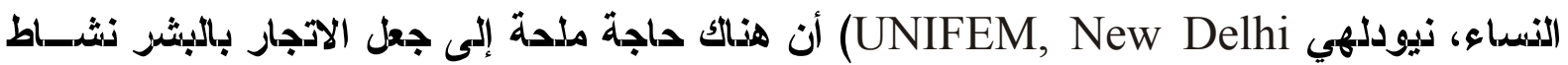

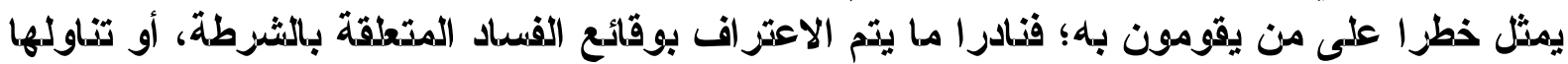

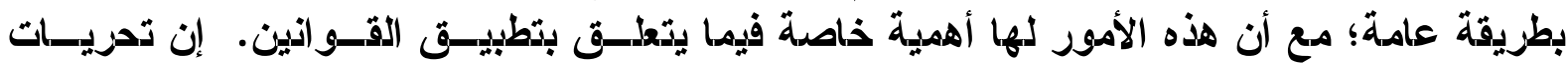

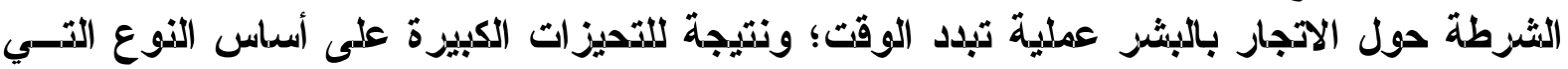

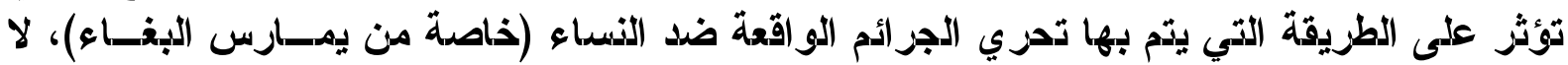

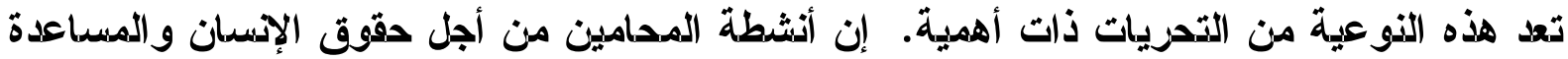

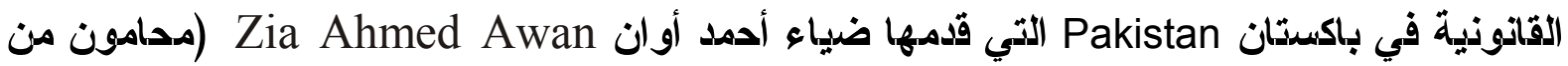

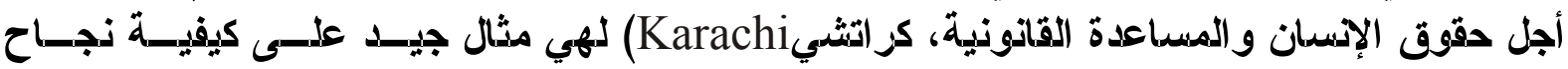

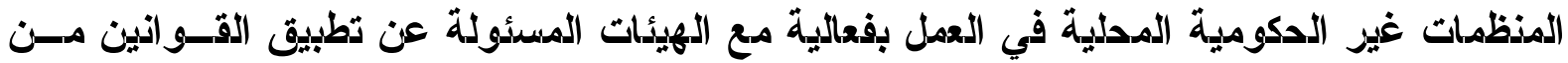

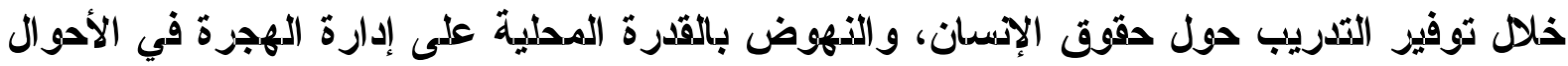

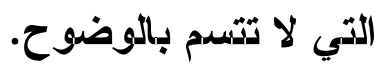

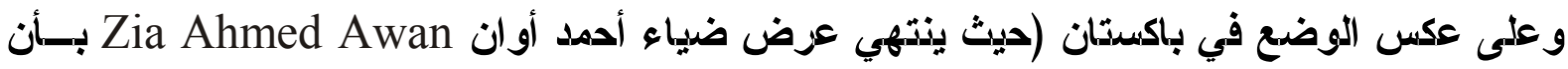

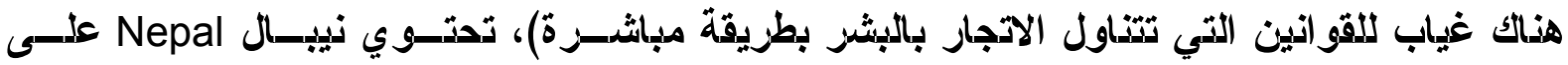

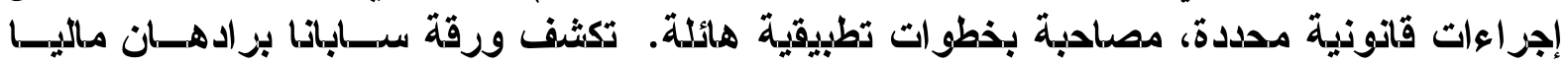

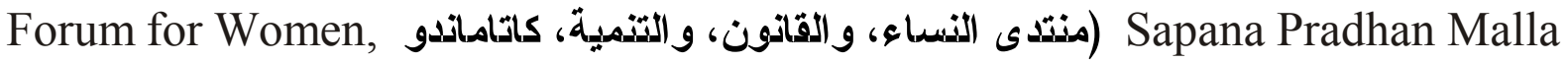
ثيال Law and Development, Kathmandu

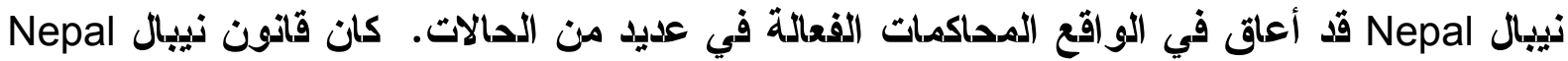

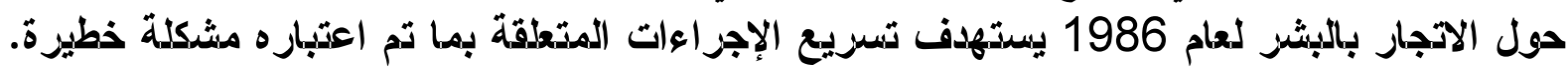

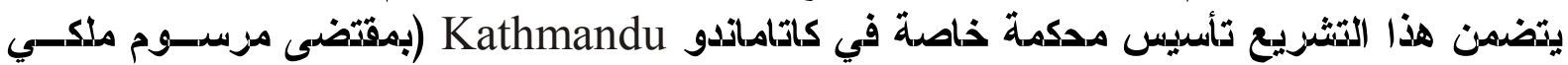

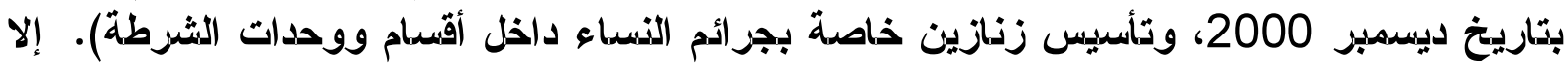

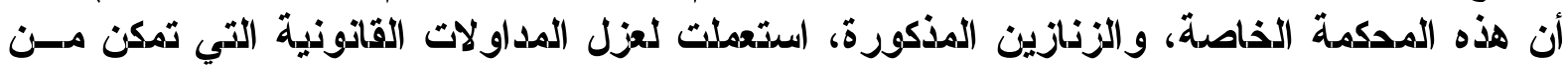

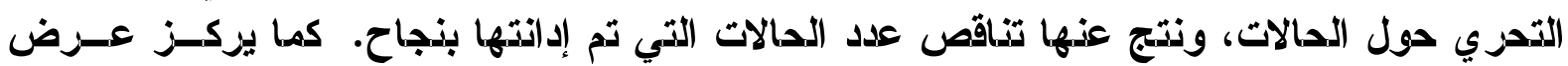

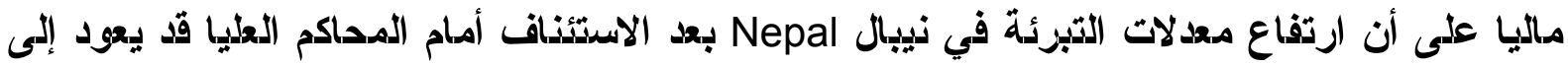

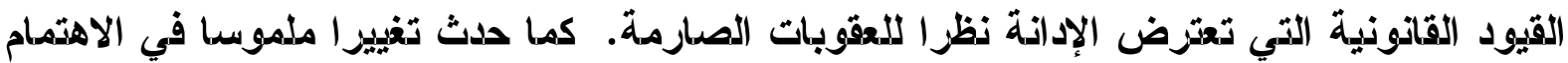

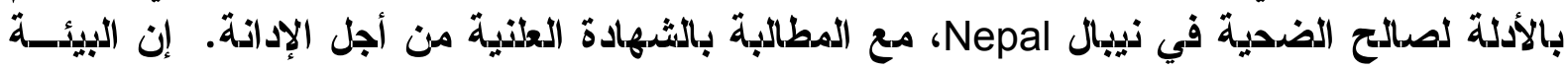

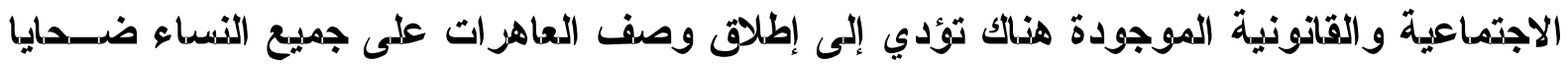

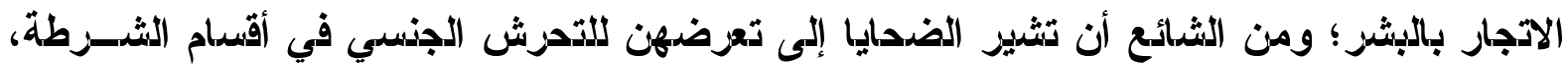

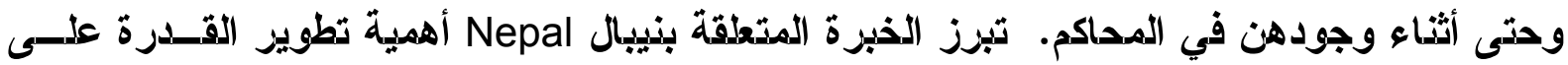

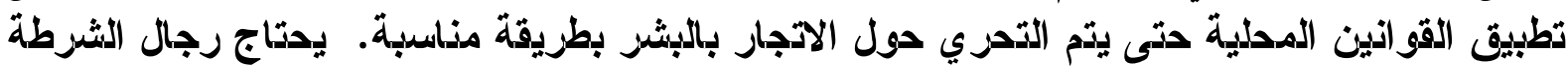

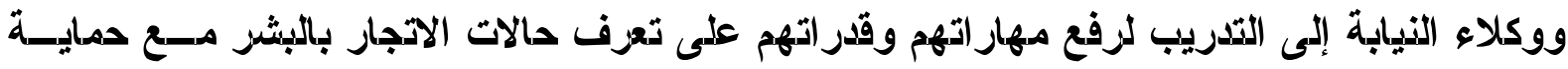

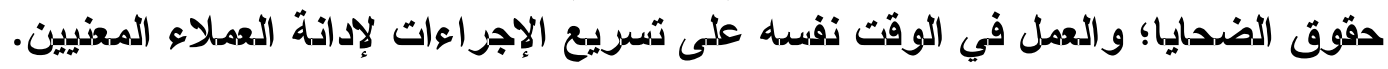

IIlegal Labor and the Commercial العمل غيسز الثـزعي والاسـتغلال التجساري للأطفسال Exploitation of Children Child Trafficking: Complexities and Coalitions الاتجار بالأطفال: التعقيد/ت والاتتلاذفات إن التعامل مع الأطفال ضدايا الاتجار مسألة تتسدم بمزيد من المباشزة، وهي أكثر تعقيدا في الوقت 


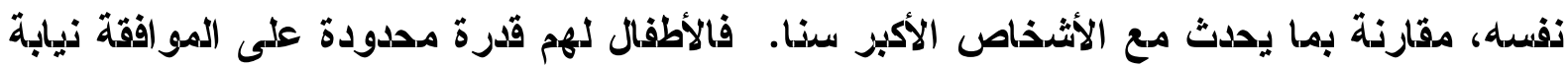

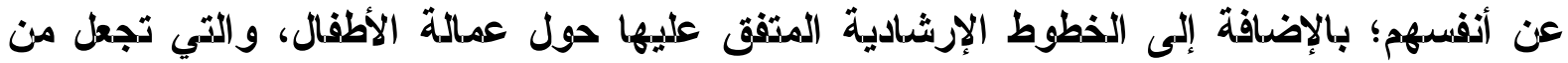

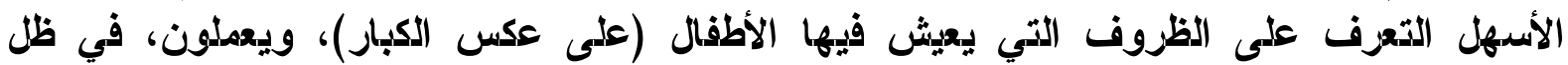

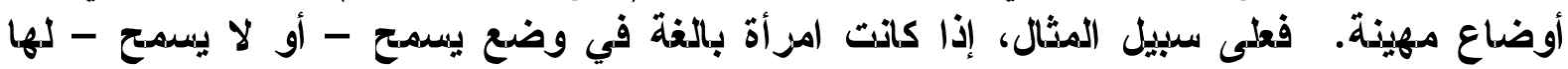

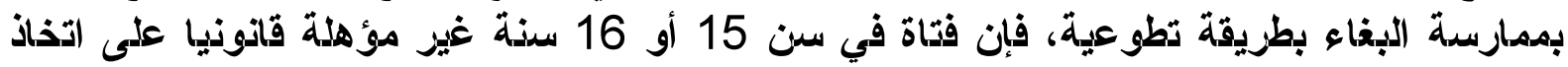

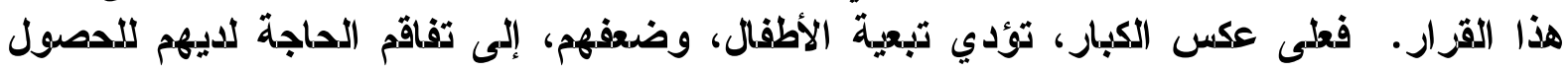

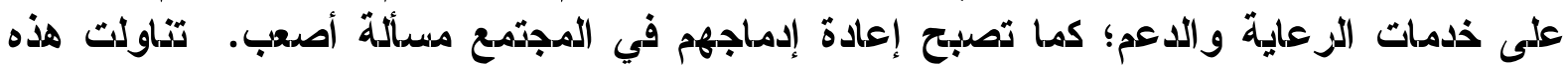

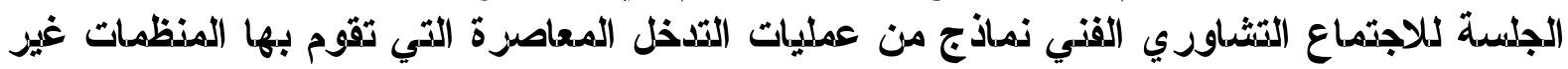

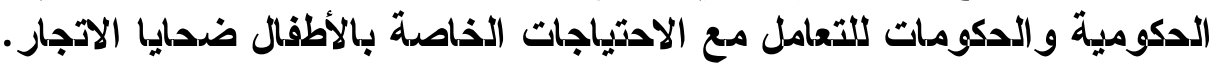

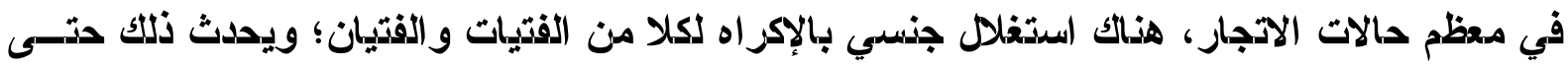

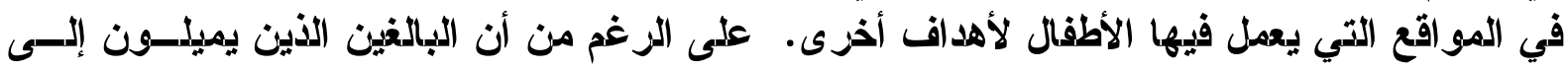

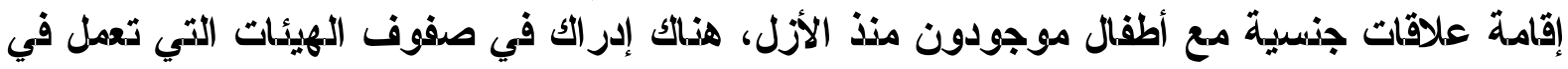

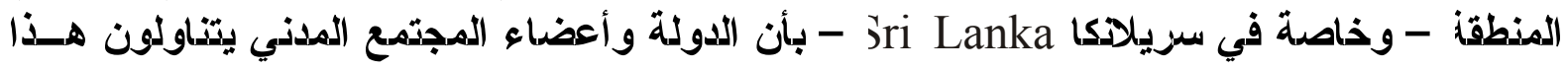

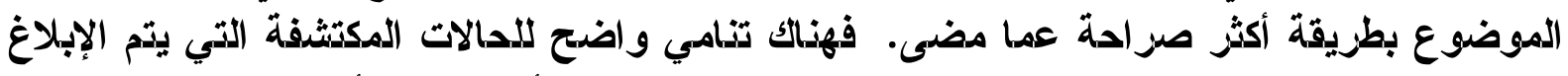

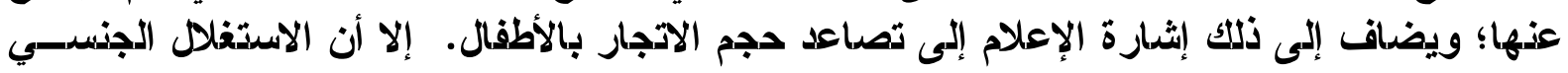

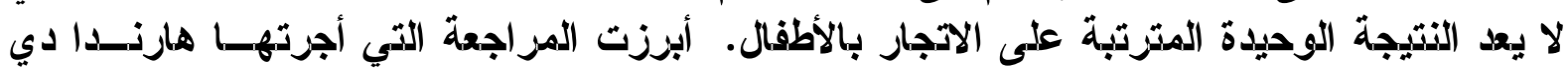

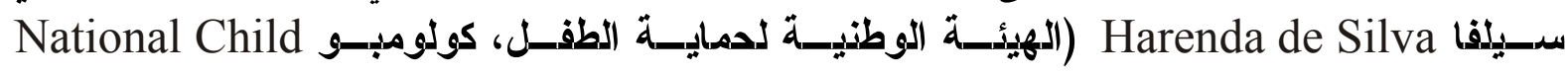
(Protection Authority,Colombo

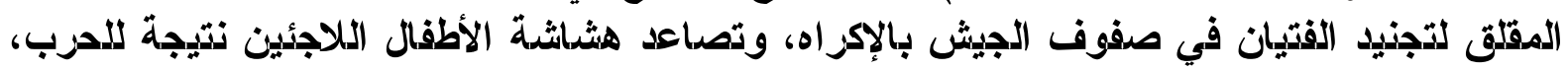

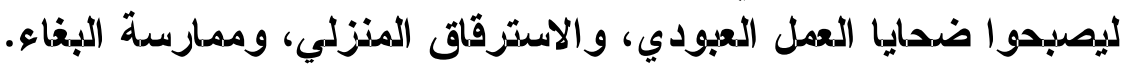

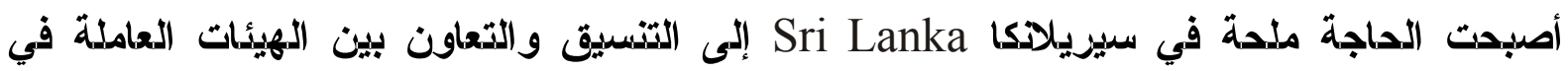

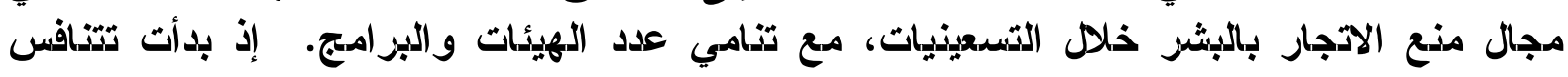

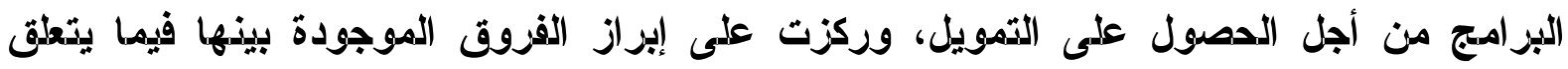

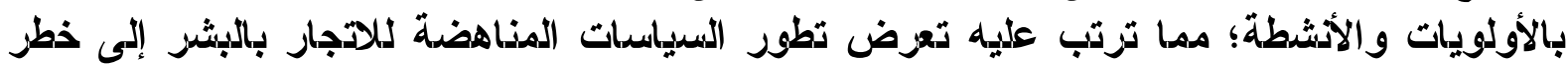

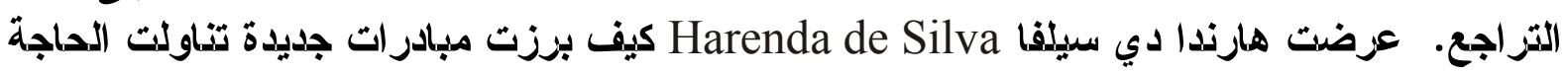

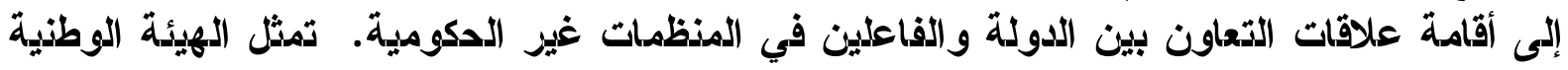

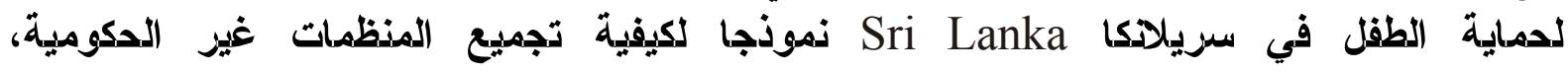

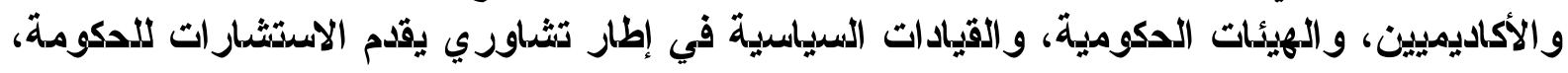

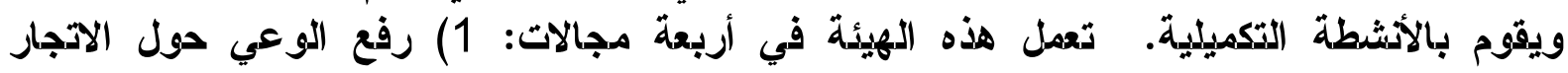

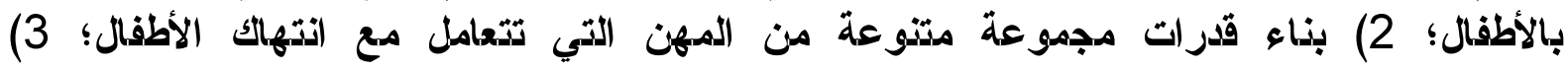

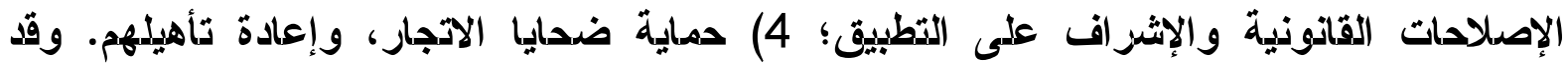

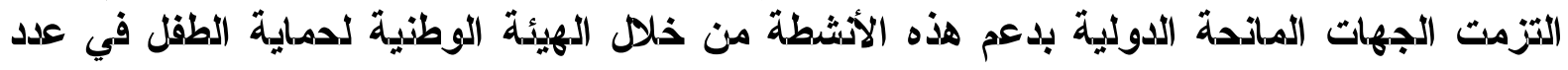

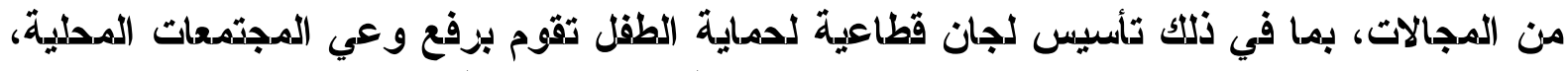

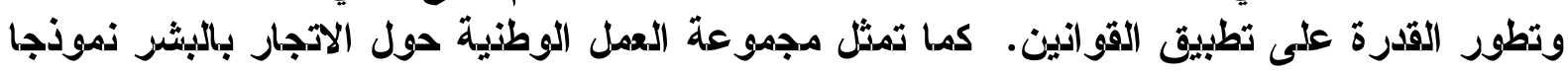

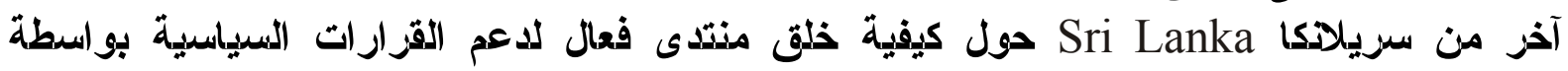
مصادر تدظى بمصداقية كبيزة. 
ميزانور رحمن Mizanur Rahman (ثببكة العمل لمناهضة الاتجار بالأطفال، واستغلالهرم جنسيا،

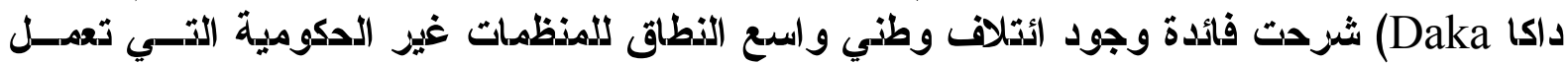

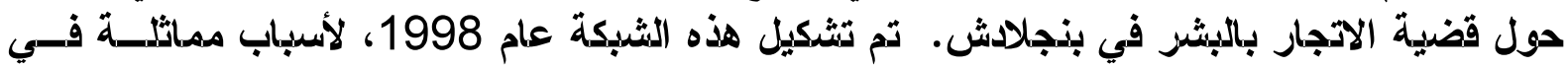

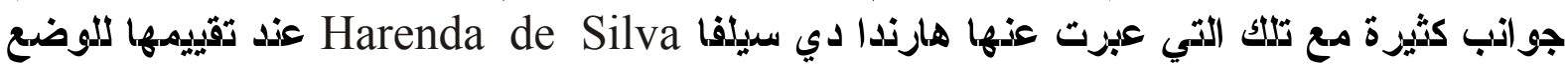

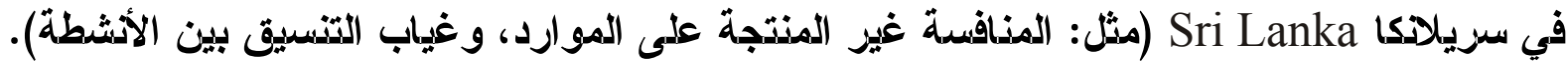

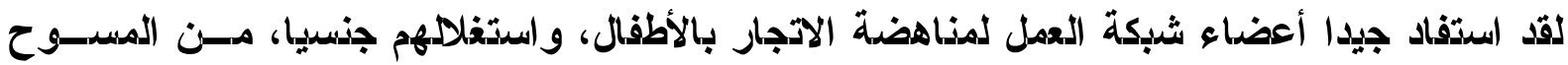

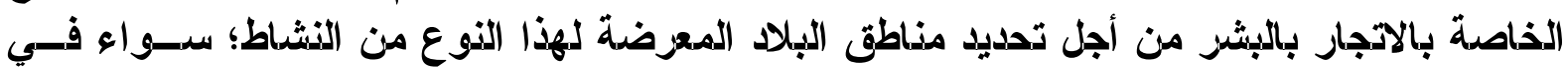

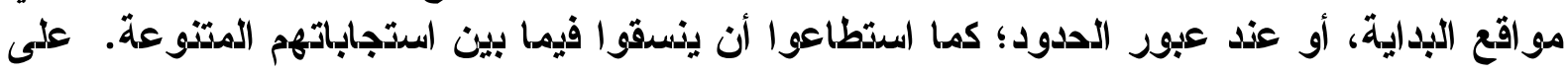

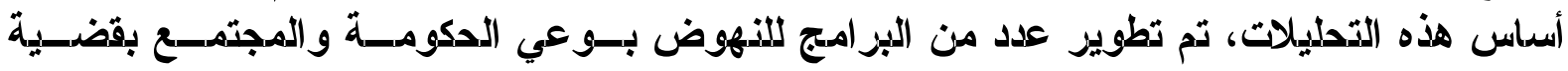

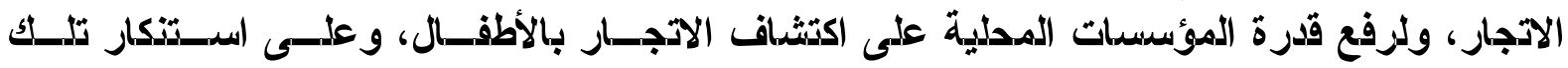

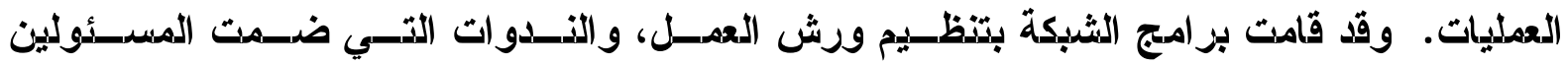

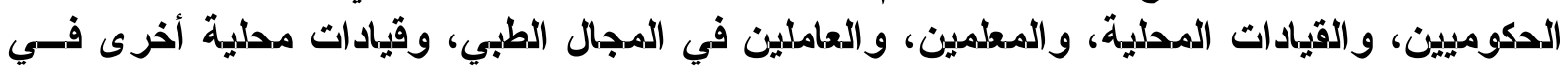

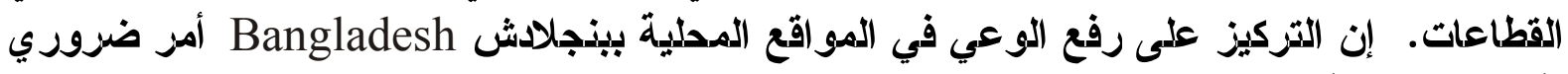

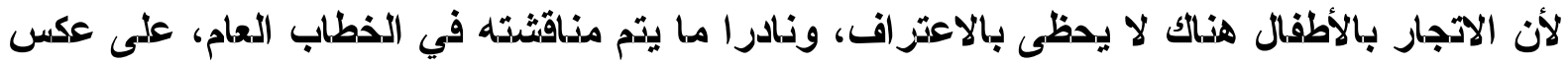

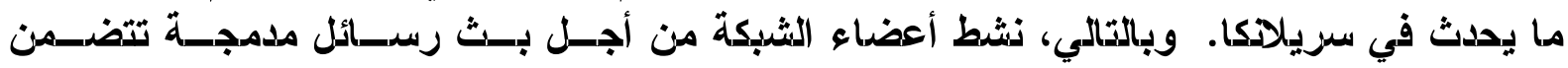

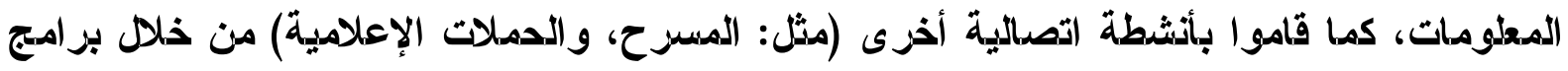

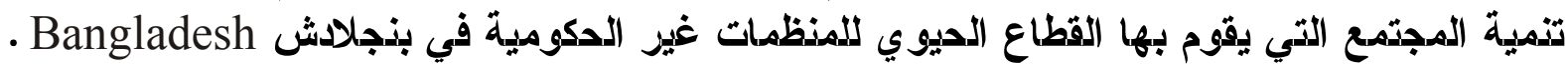

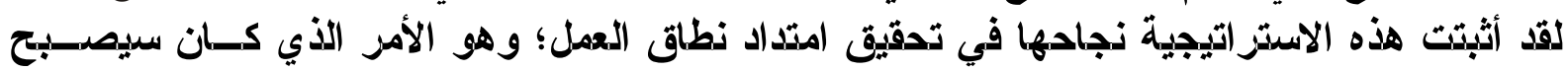
مستحيلا لو قامت به منظمة واحدة.

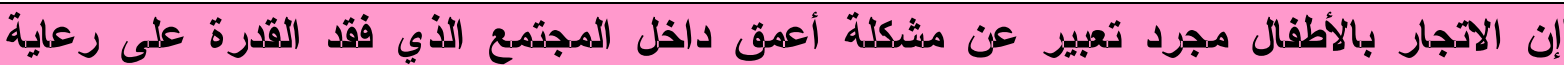

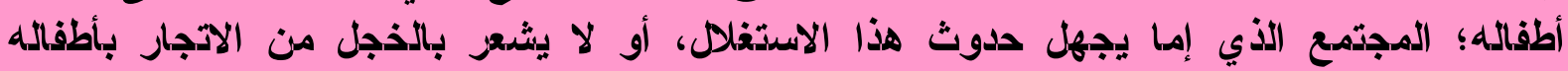

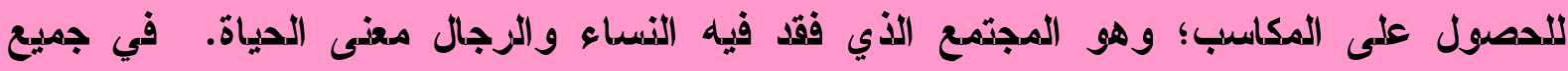

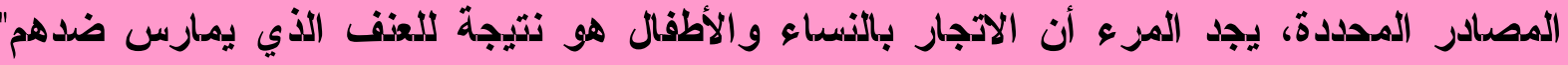
.(Indrani Sinha and Roop Sen, page 6)

تناولت أيضا ورقة كل من إندراني سينها Indrani Sinha وروب سين Roop Sen (إكبات، كلكتا (ECPAT, Calcutta

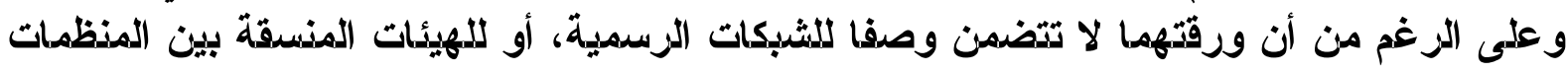

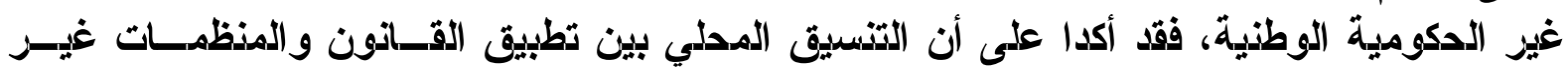

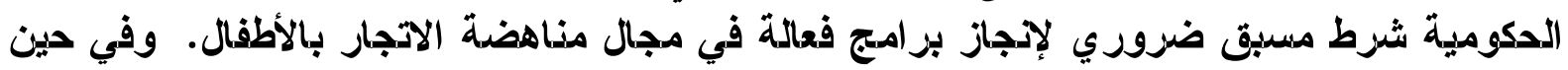

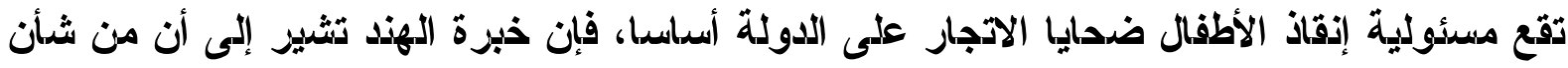

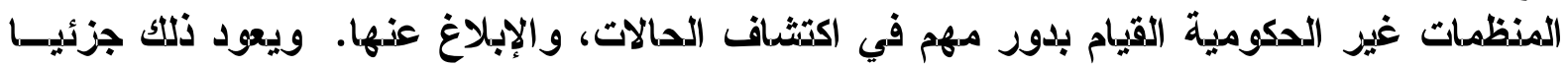

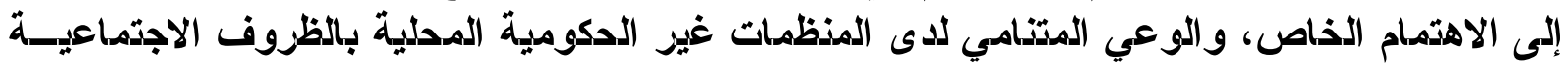

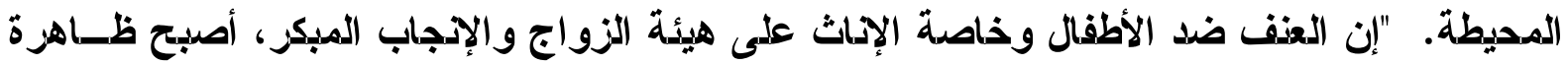

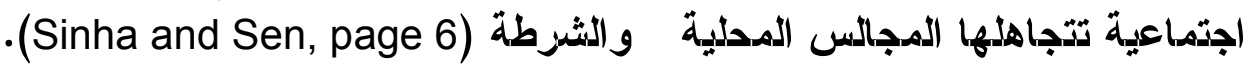


بعد انتثال الأطفال من الأماكن التي يتم احتجازهم فيها، تتمتع المنظمات غير الدكومية باستعدادات

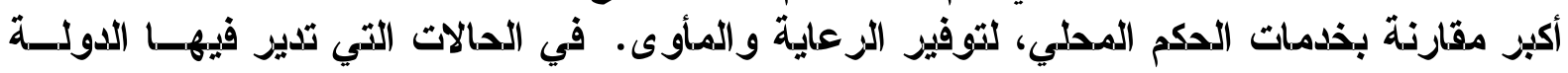

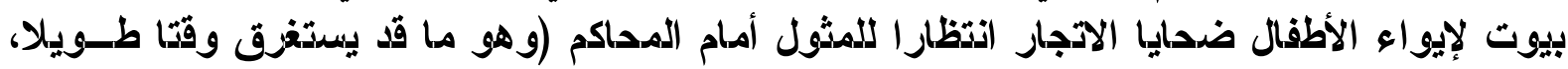

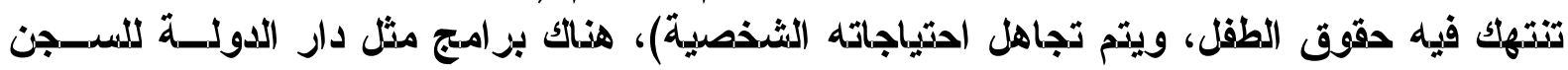

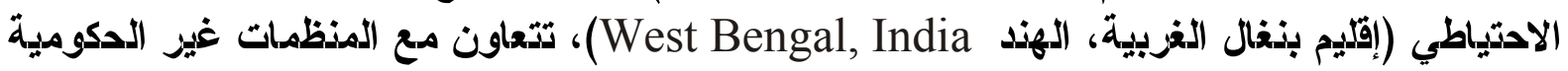

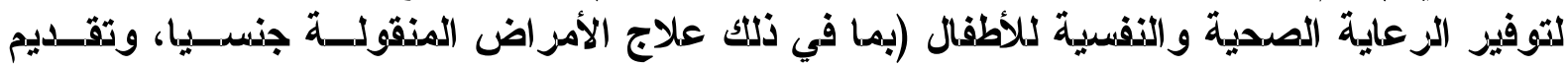

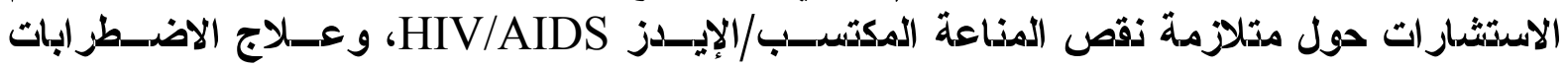

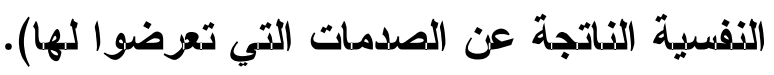

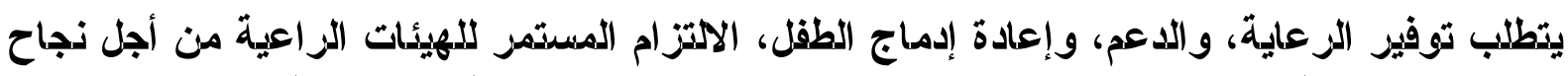

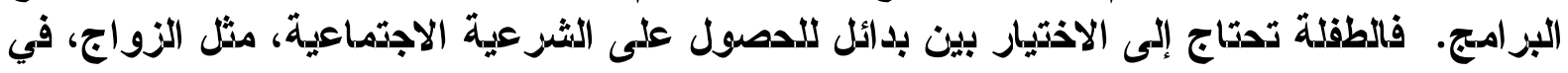

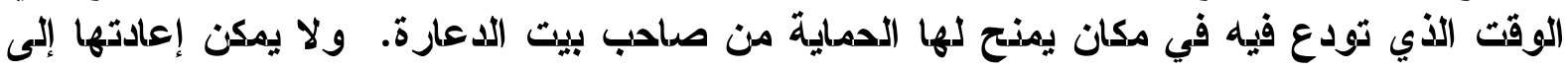

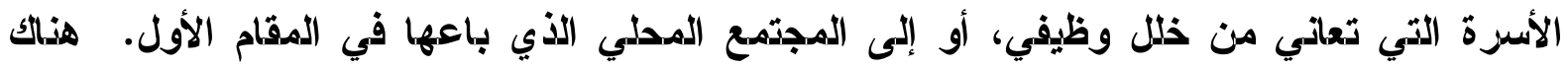

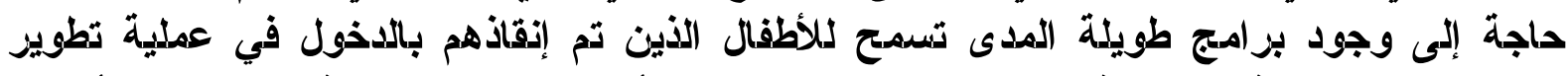

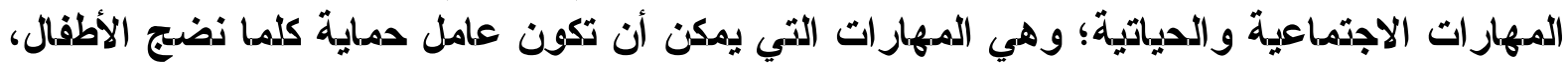

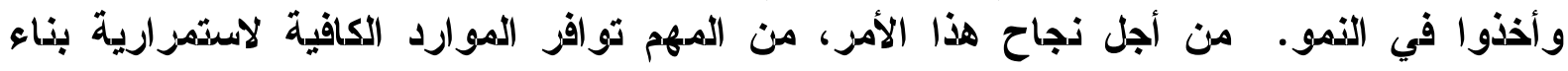

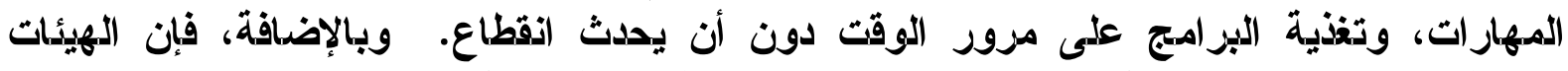

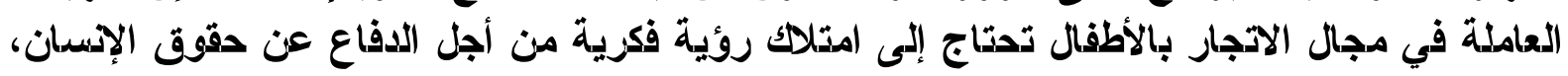

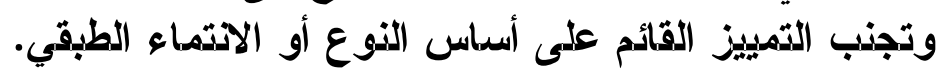




\section{IDENTI FYING PROGRAM EVALUATION تديد منهجيـات تقيسيم البـرامج METHODOLOGI E S}

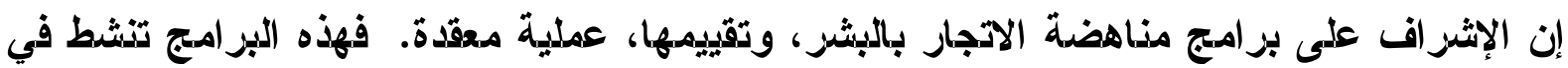

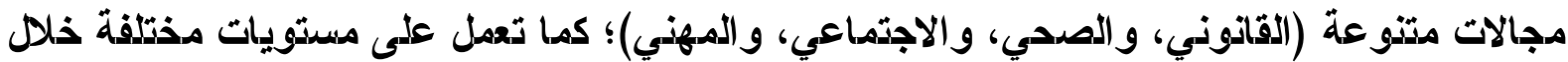

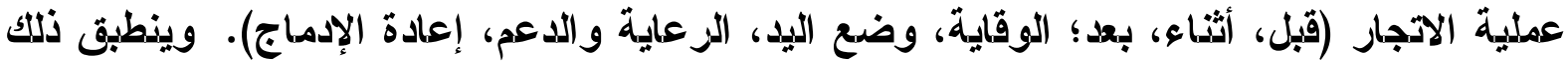

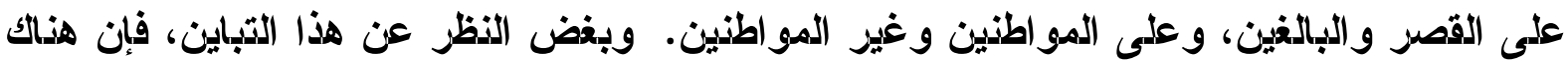

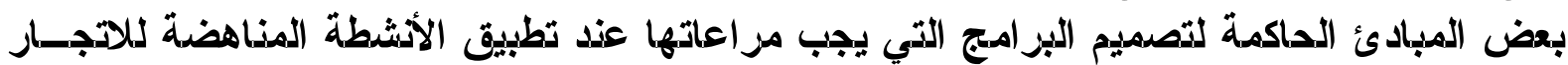

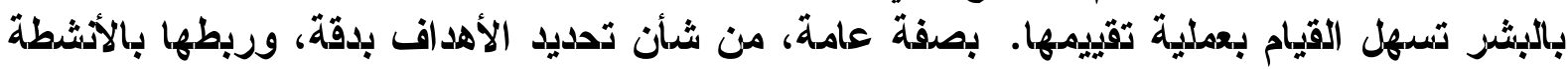

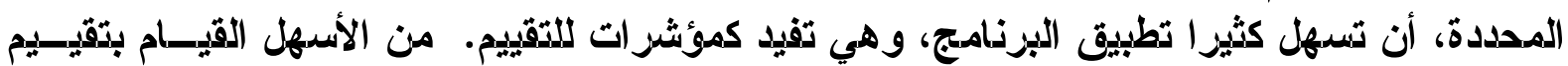

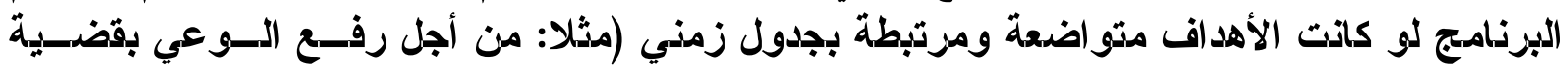

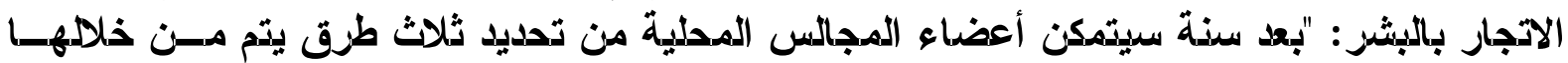

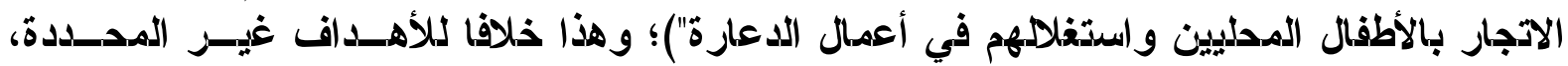

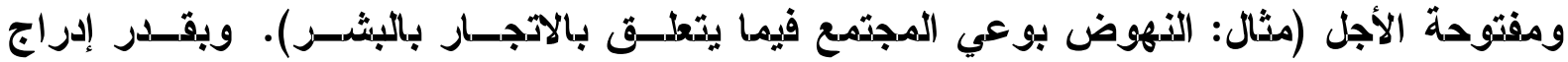

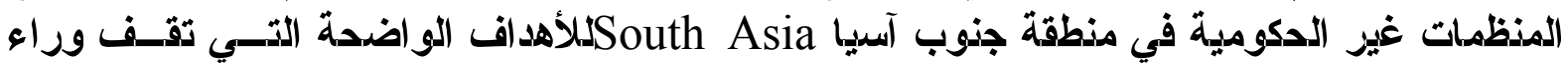

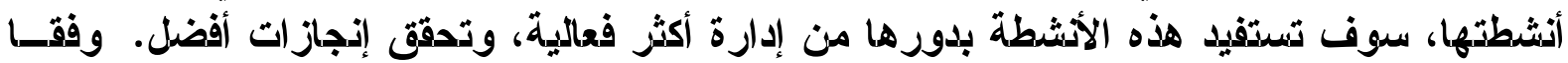

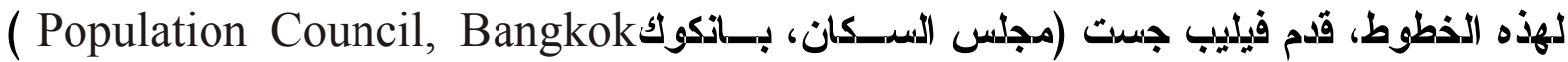

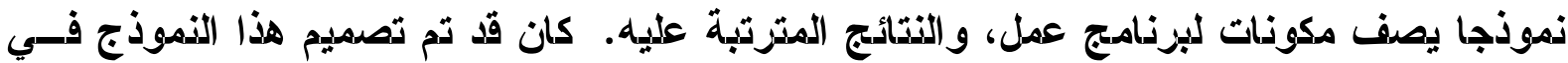

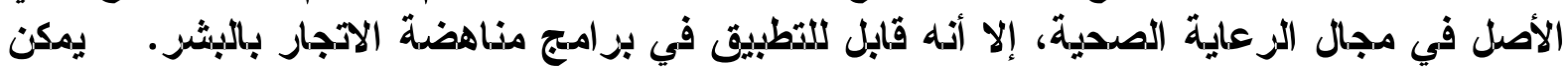
تلذيص هذا الإطار الذي يشرح السبل المتعلقة بالمدخلات، والعمليات، والمخرجات، والئ والنتائج، فيمسا يلي:

$$
\text { التعريف المدخلات، والعمليات، والمذرجات، والنتائجج }
$$

:Process العمليات

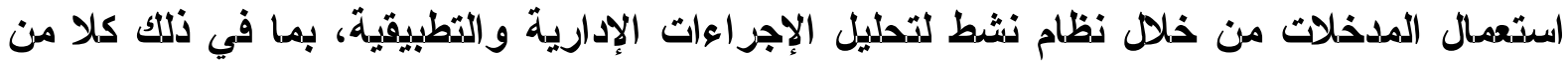

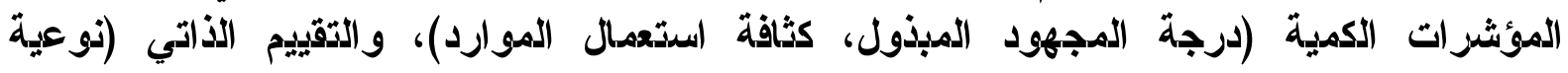
التطبيق، مدى التكيف مع التغييزات التي تددث في البيئة، نمط الإدارة، استجابات الثردات الثركاء).

المخزجات Output بياتات كمية تثيز إلى أداء البزنامج (مثال: الأشطة التي تم القيام بها، والخدمات المستعهلة، و الفئات (المستفيدة، وتوزيع السئ العلع).

النتائج Outcome: يتم استُعمال نوعان من النتائج: النتائج طويلة الأمد (مثال: إعادة الإدماج الناجج للعائدين من ضدايا 


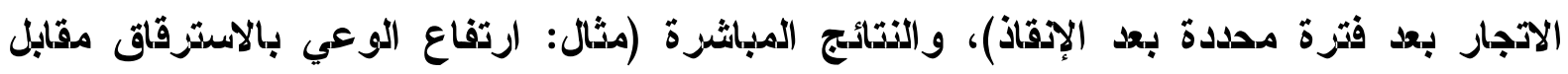

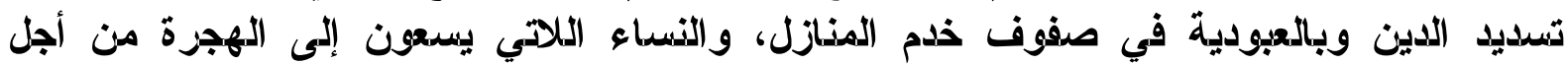

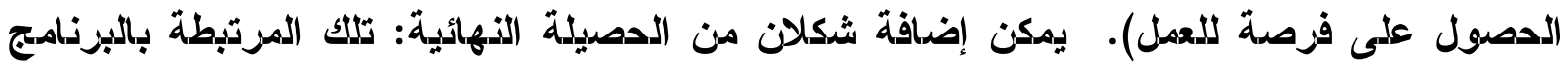

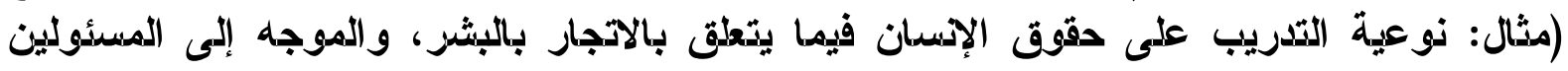

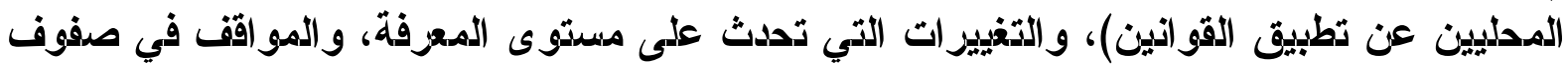

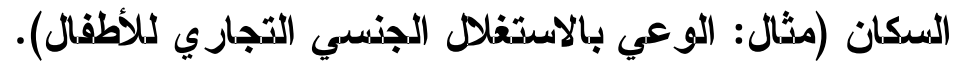

\section{- مذهجيات المتابعة والتقييم Monitoring and Evaluation Methodologies-}

نظرا لتعدد عناصز البزامج)، ولتذوع المجموعات السكانية المندرجة في برامج مناهضة الهية الاتجار

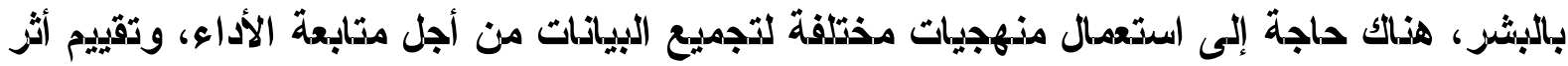

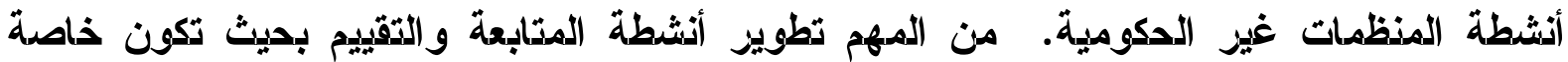

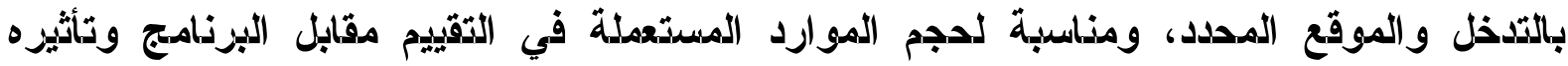

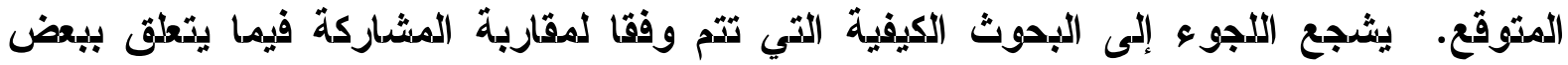

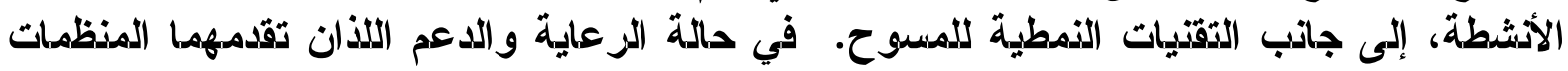

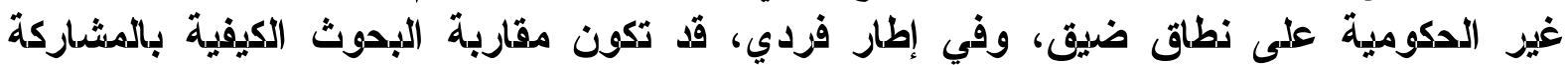

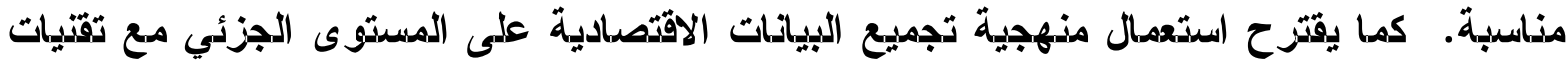

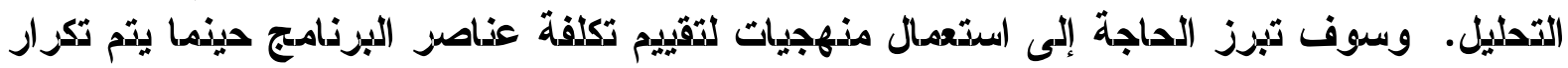
عمليات التذخل الذاجدة، وتوسيع نطاق تطبيقها.

يقدم الجدول التالي فكرة عامة حول مبدأ منهجيات تجميع البياتات، و المقاربات الاعامة لتصميم تقييجم

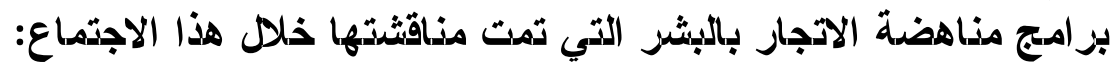
نظرة عامة حول منهجيات المتابعة والتقييم

\begin{tabular}{|c|c|}
\hline منهجية البحث & المؤشر//الحجم \\
\hline 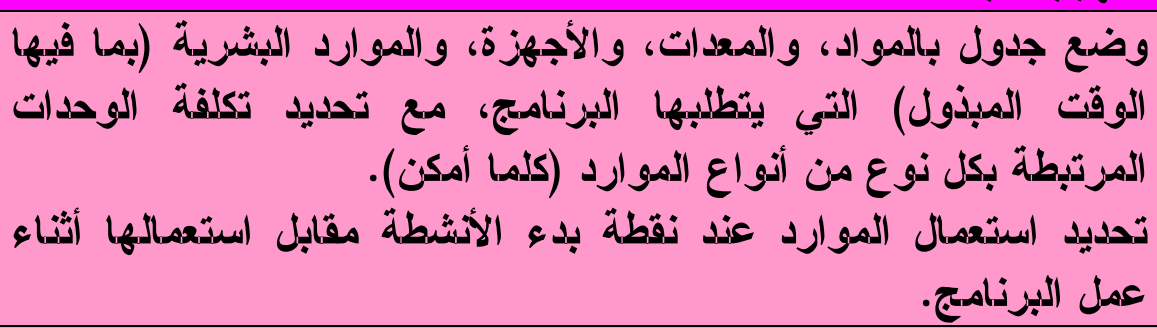 & المدخلات \\
\hline 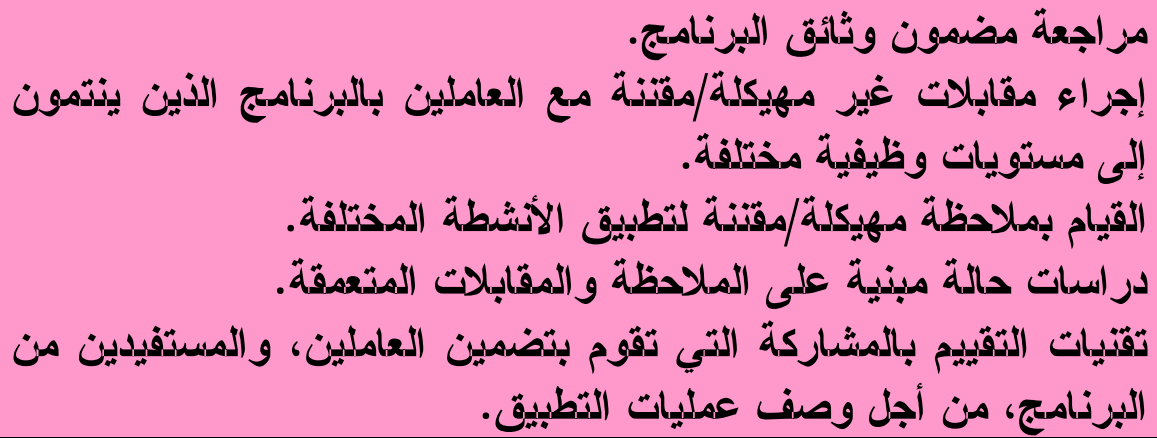 & $\begin{array}{r}\text { العمليات } \\
\text { Process }\end{array}$ \\
\hline
\end{tabular}




\begin{tabular}{|c|c|}
\hline منهجية البحث & المؤشثز/الحجم \\
\hline 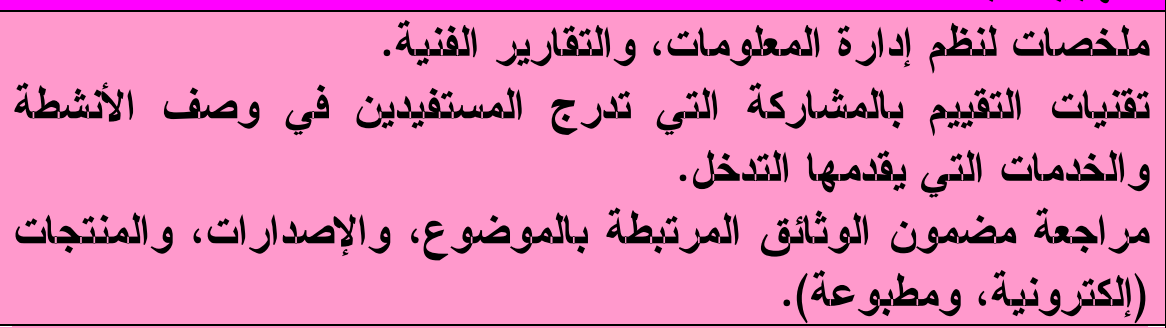 & المخرجات \\
\hline 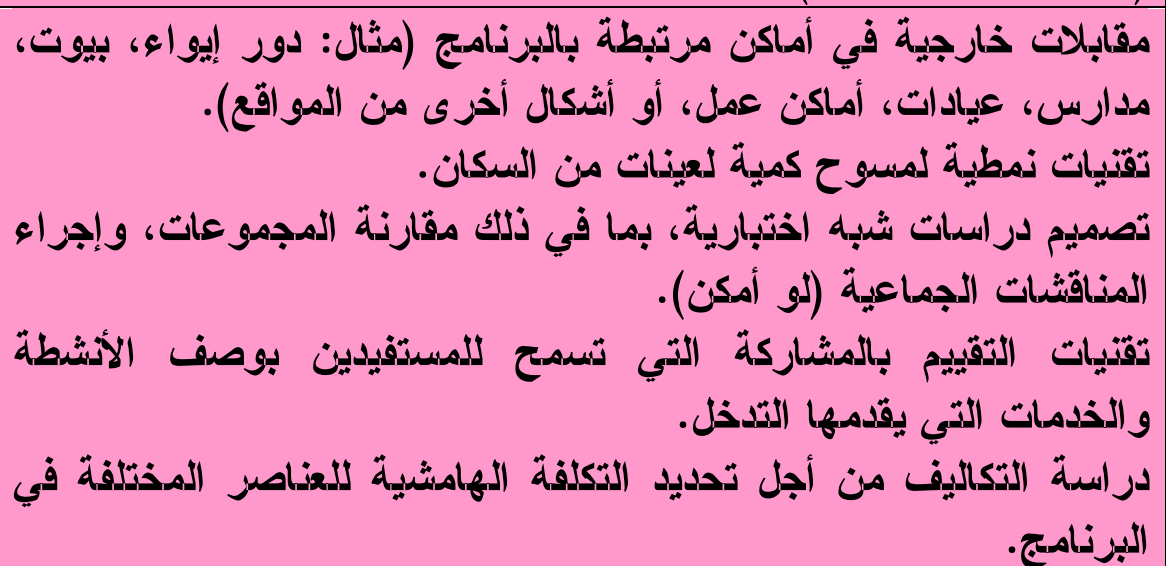 & $\begin{array}{l}\text { النتائج متوسطة الأجل } \\
\text { Outcome: Intermediate }\end{array}$ \\
\hline 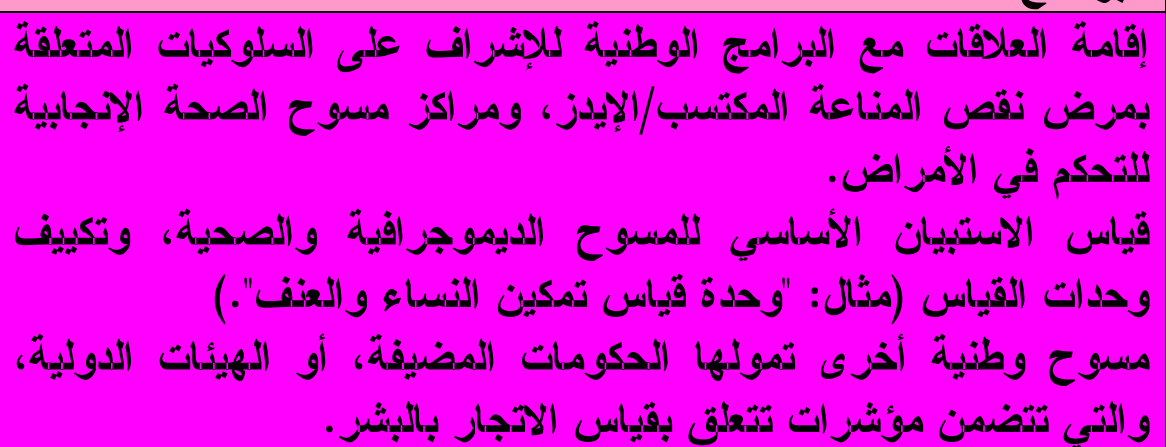 & $\begin{array}{c}\text { النتائج طويلة الأجل } \\
\text { Outcome: Long- } \\
\text { Term }\end{array}$ \\
\hline
\end{tabular}

-

لم يتم بعد اختبار الإطار المفاهيمي لتقييم برامج مناهضة الاتجار بالبشر، والهذهجيات البحثية

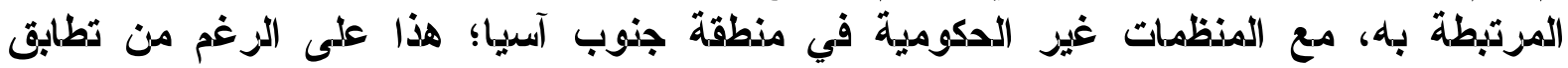

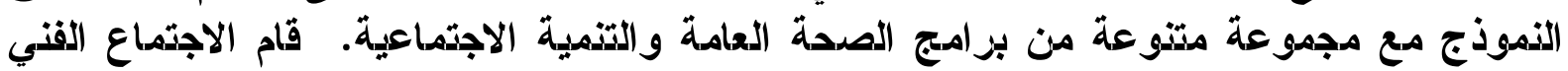

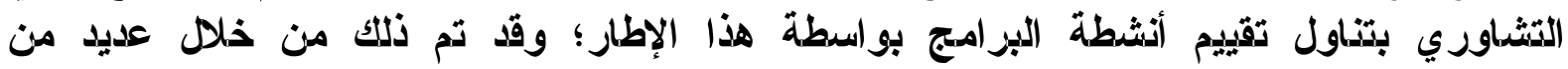

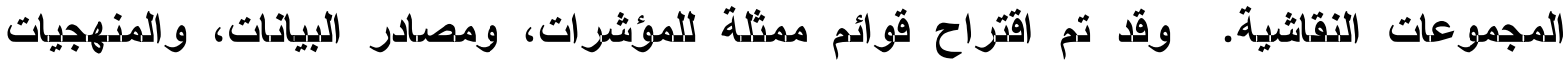

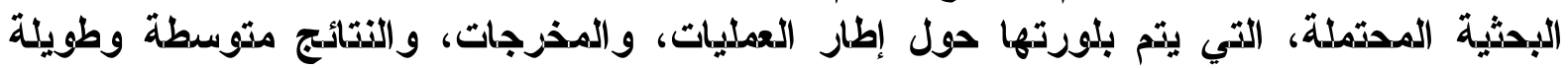

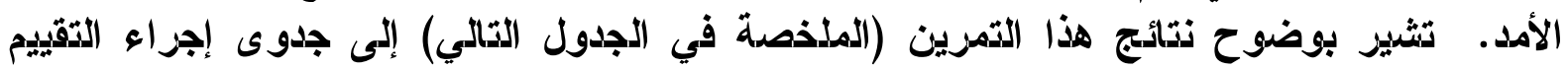

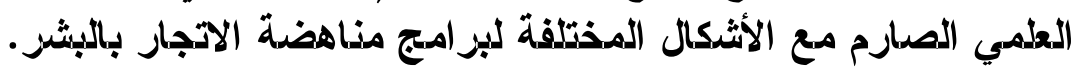




\section{قائمة توضيدية لعينة تتعلق بتقييم برنامج}

\begin{tabular}{|c|c|c|c|c|}
\hline \multirow{2}{*}{ منهجية البحث } & \multirow{2}{*}{ دصدر البياتات } & \multirow{2}{*}{ المجموعة المرجعية } & \multicolumn{2}{|r|}{ المؤشر } \\
\hline & & & المستوى & التعريف \\
\hline تحليل المضدون & ميز انيات حكومية & خدومية & العمليات & الذصيص الدملة الموارد الذدمات \\
\hline منسجيح $\quad$ مالمشاركة & الذين الديع المقيمين مندف & 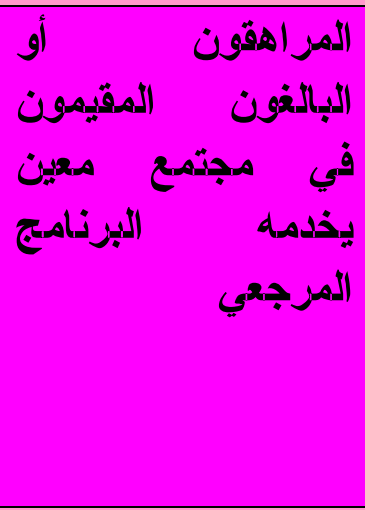 & المخرجات & 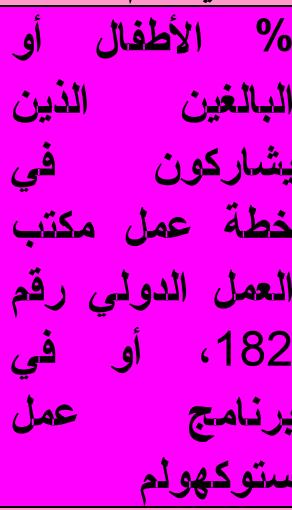 \\
\hline 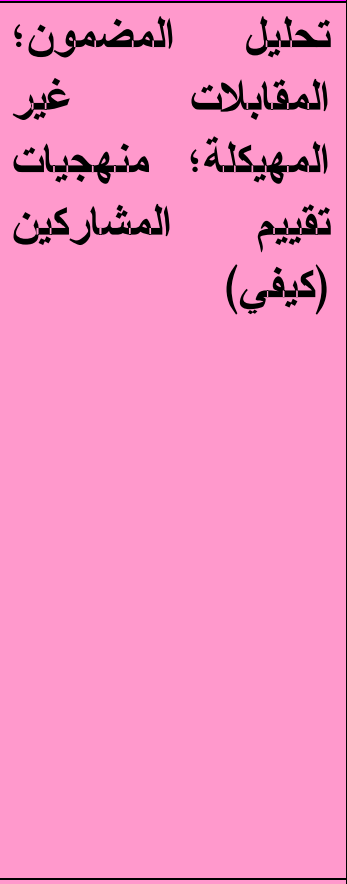 & والتدريبي $\quad$ البزنامج & 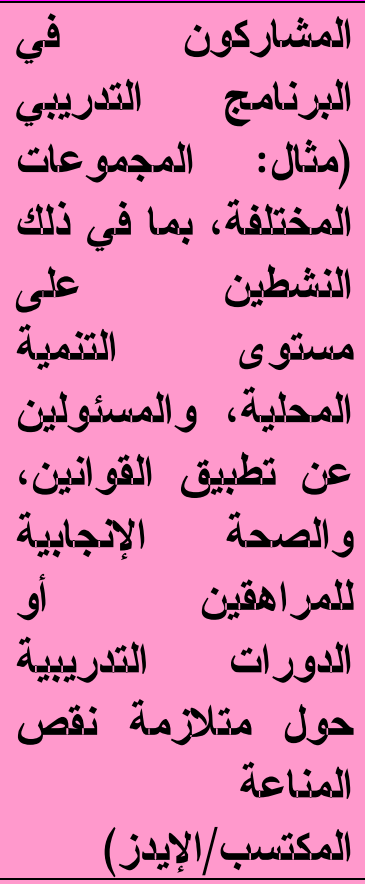 & العمليات & التضمن \\
\hline 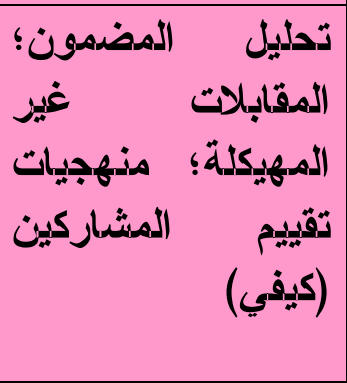 & 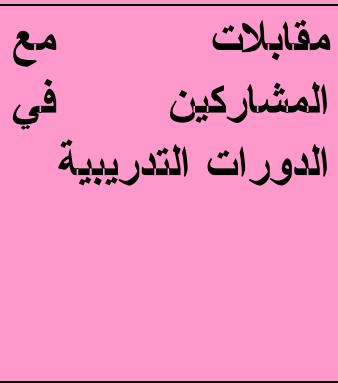 & 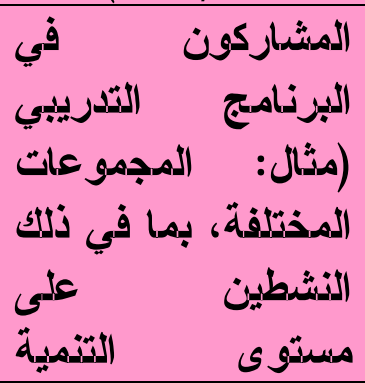 & المخرجات & 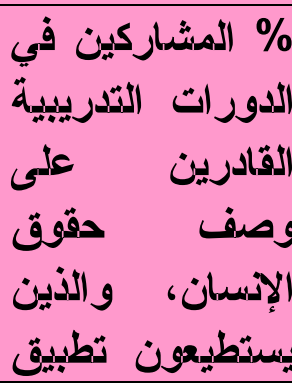 \\
\hline
\end{tabular}




\begin{tabular}{|c|c|c|c|c|}
\hline \multirow{2}{*}{ منهجية البحث } & \multirow{2}{*}{ مصدر البيانات } & \multirow{2}{*}{ المجموعة المزجعية } & \multicolumn{2}{|r|}{ المؤشر } \\
\hline & & & المستوى & التعزيف \\
\hline & & 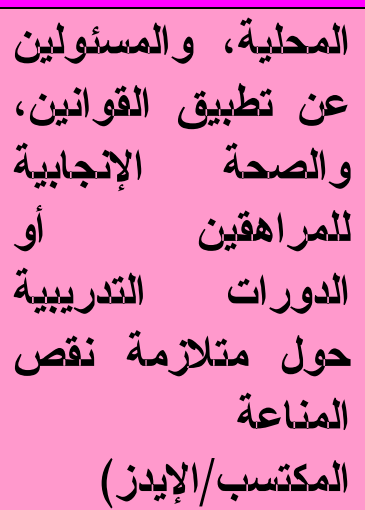 & & 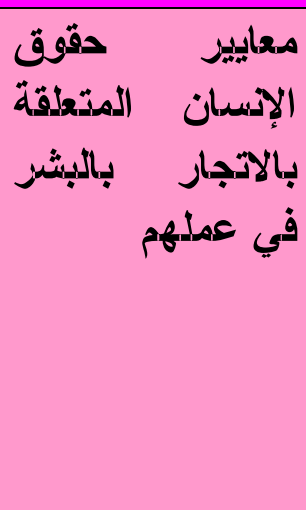 \\
\hline 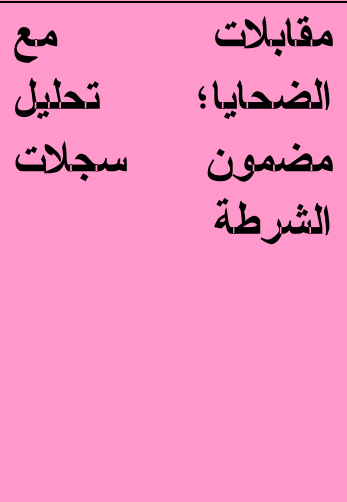 & سجلات الثرطة؛ & تطبيق القواتين & مباشز & 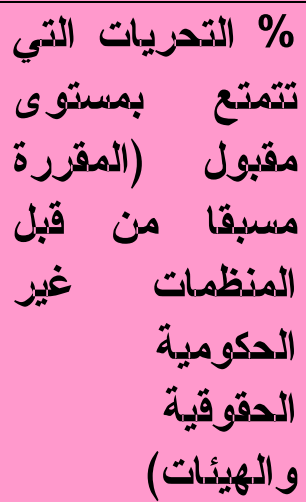 \\
\hline تحجلات الشرطة & سجلات الثرطة & التي يتم الإبلاغ عاتهار بالبشر & مخز مخز & 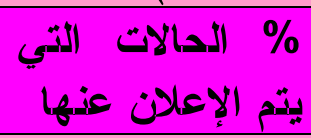 \\
\hline 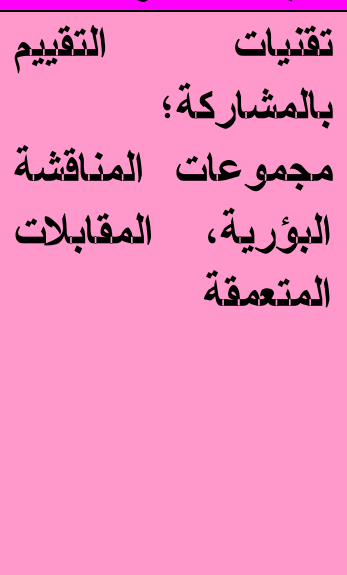 & الائات الأفزد الذين يمثلون & 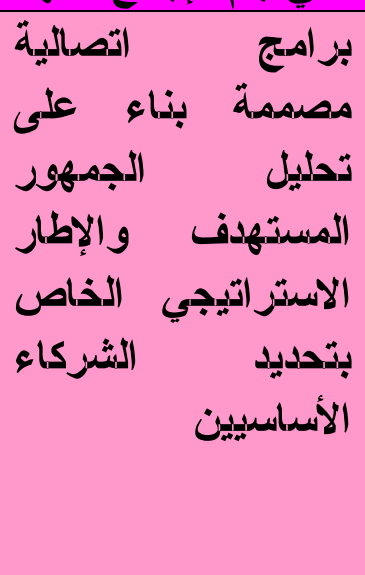 & الإعمليات & 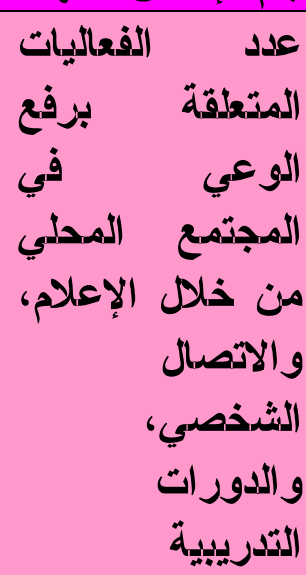 \\
\hline المسح بالعيذة & 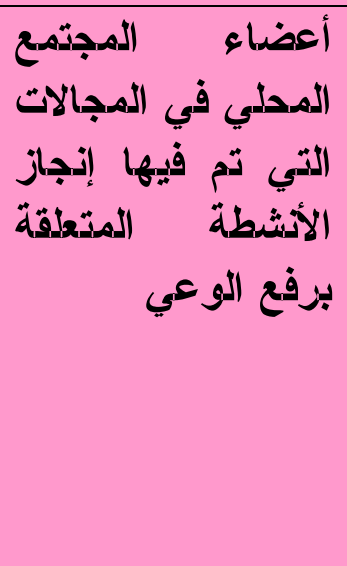 & 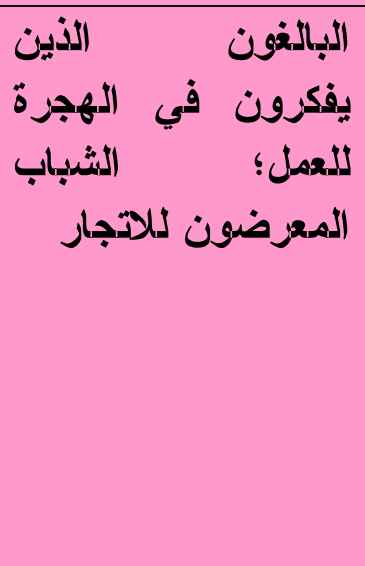 & مخزج & 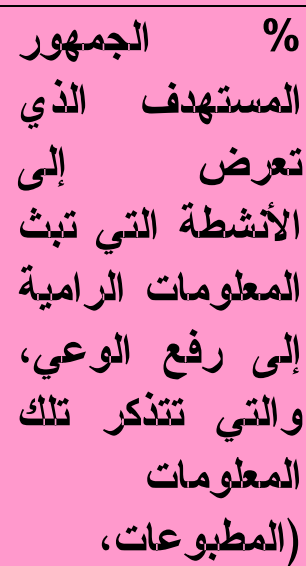 \\
\hline
\end{tabular}




\begin{tabular}{|c|c|c|c|c|}
\hline \multirow{2}{*}{ منهجية البحث } & \multirow{2}{*}{ هصدر البيانات } & \multirow{2}{*}{ المجموعة المزجعية } & \multicolumn{2}{|r|}{ 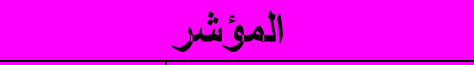 } \\
\hline & & & المستوى & التعريف \\
\hline & & & & (المسرح، الإعلام) \\
\hline للقيادات الأساسية بالعينة & المدلي & 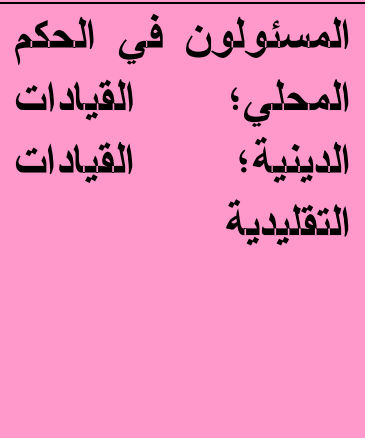 & مخز مبز & 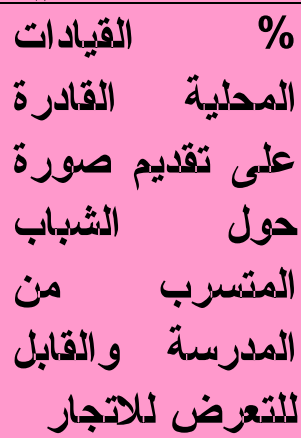 \\
\hline بالمشاريات & 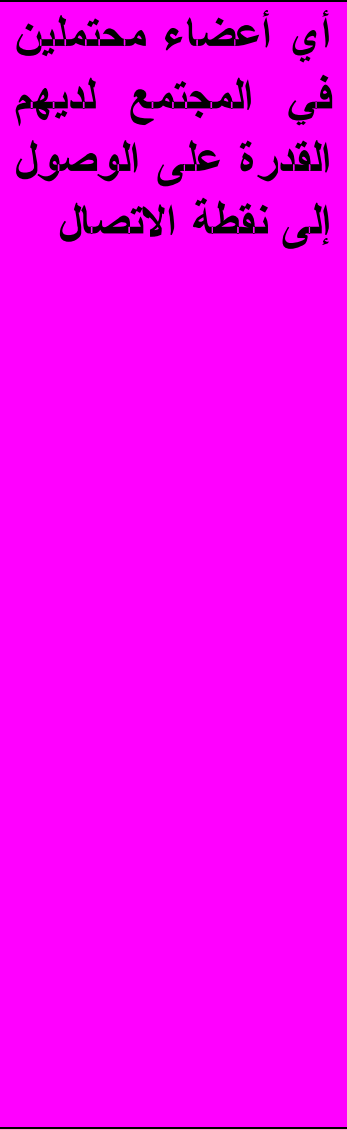 & تالمباء & مبز مبز & 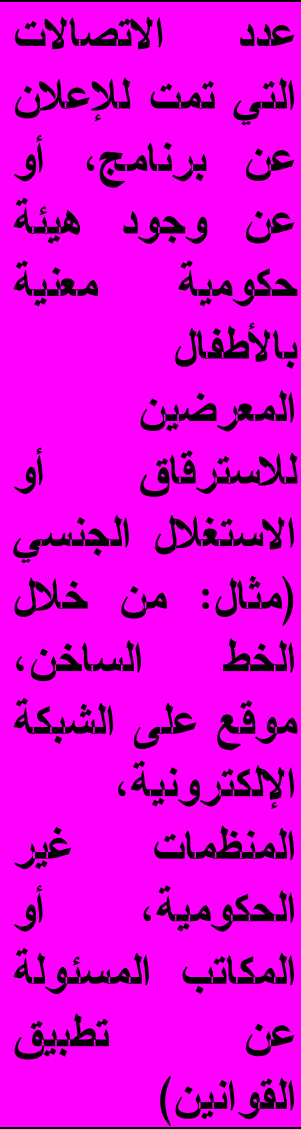 \\
\hline مضمون & التقاريز الناتجة عن & ضدايا الاتجار بالبشر & مخزج & عدد الأطفال \\
\hline البز امج؛ & البزامج؛؛ المقابلات & الذين تم التعزف & متوسط & والبالغين الذين تم \\
\hline منهجيات التقييم & مع مستهدفين & عليهم، إنقاذهم & 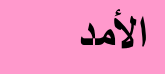 & انتشالهم من مكان \\
\hline بالمشاركة؛ & مختارين & بواسطة برنامج مبني & & الاستغلال (مثثال: \\
\hline المقابلات المتعدقة؛ & قصدية & مقاربة & & الاسترقاق \\
\hline دراسات الحالة & ل & المشاركة & & الالمجمدل، الأسمري، \\
\hline
\end{tabular}




\begin{tabular}{|c|c|c|c|c|}
\hline \multirow{2}{*}{ منهجية البحث } & \multirow{2}{*}{ مصدر البيانات } & \multirow{2}{*}{ المجموعة المرجعية } & \multicolumn{2}{|r|}{ المؤشر } \\
\hline & & & المستوى & التعريف \\
\hline & & & & تيزقيض آنعاض آذرين لم \\
\hline لدالمشياركة & المتئفيدين & ترنم خدمتهم من الاتجار الذين & مخز مخرط & الآتجار \\
\hline مالمشهاركة؛ & مستفيدين & ضدتايا الاتجار الذين & طخرج & عدد من تم إعادة \\
\hline لدرابدات المالة المتعدقة؛ & 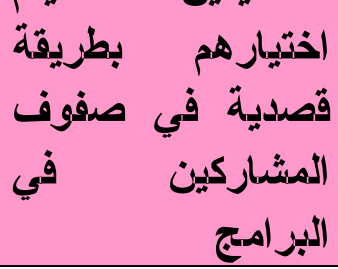 & بزنامج بالمشاركة & الأمد & 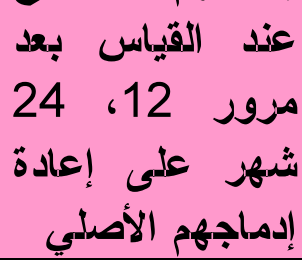 \\
\hline
\end{tabular}




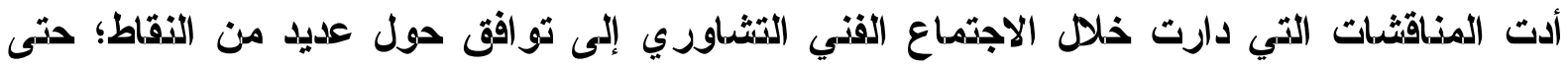

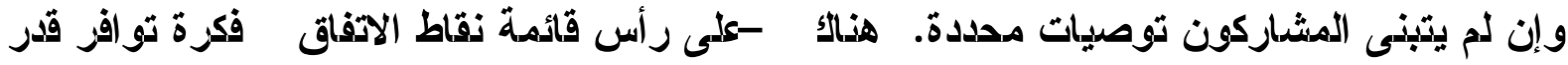

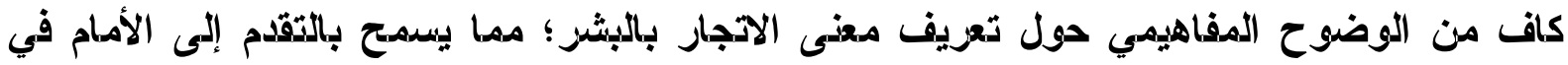

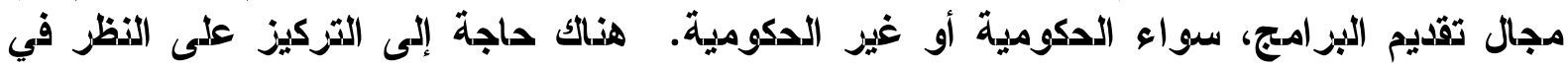

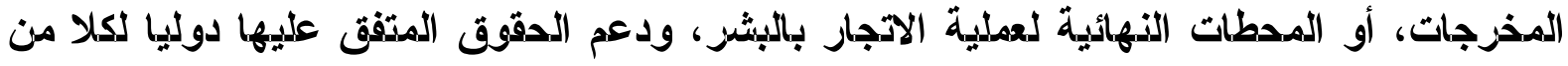

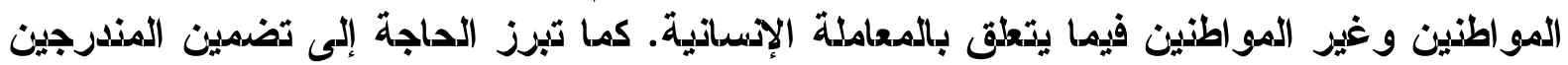

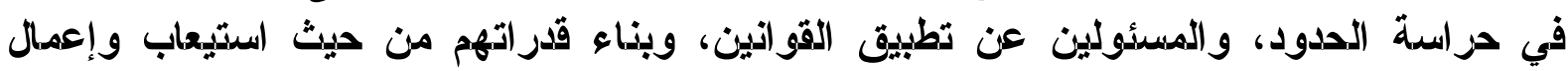

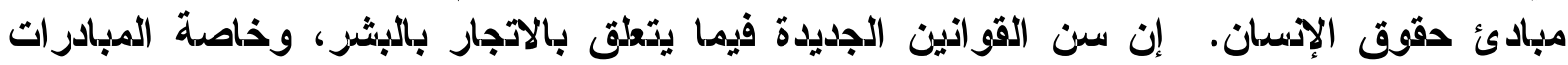

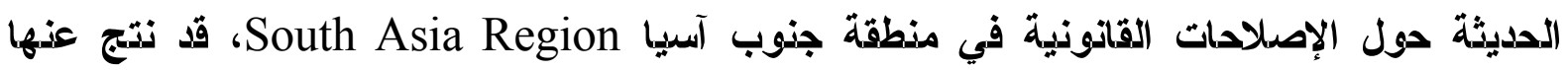

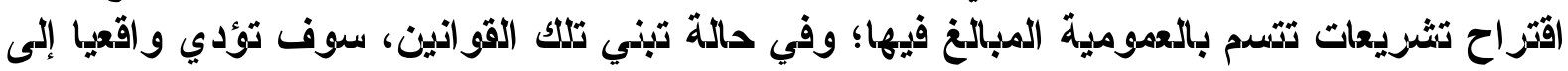

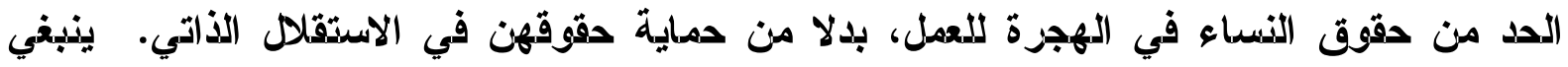
تطويز القوانين الدالية، وتطبيقها بطريقة مذصفة.

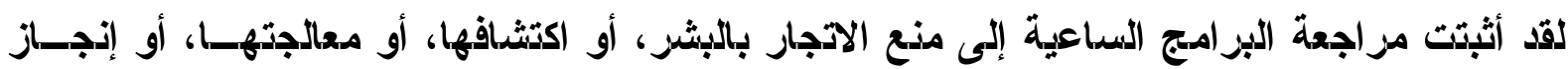

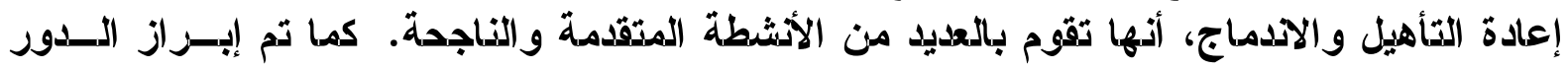

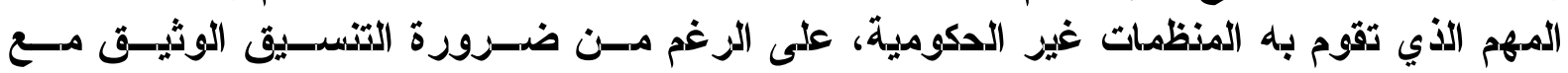

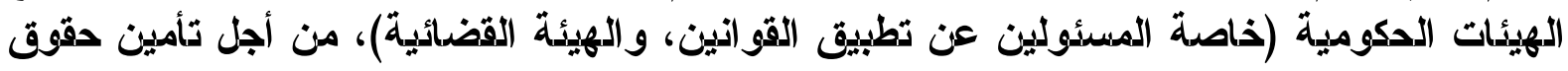

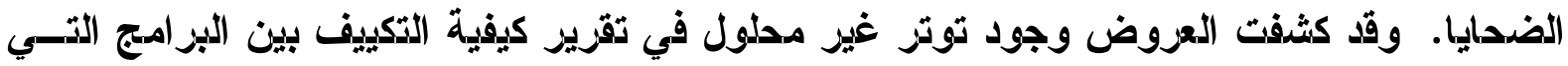

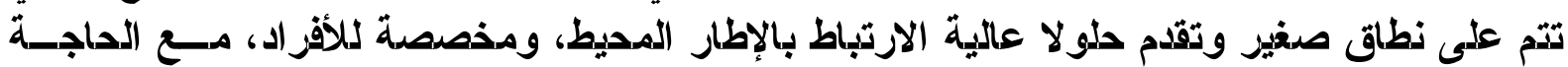

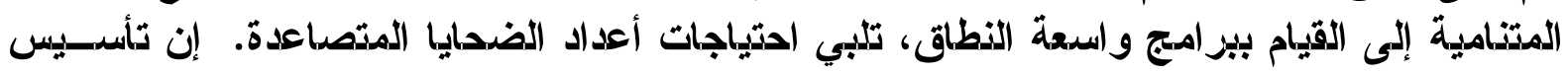

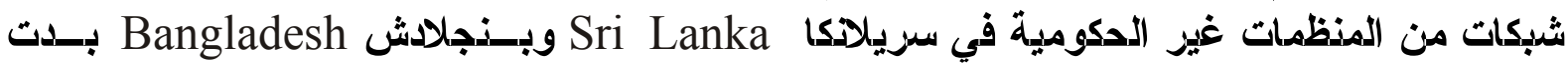

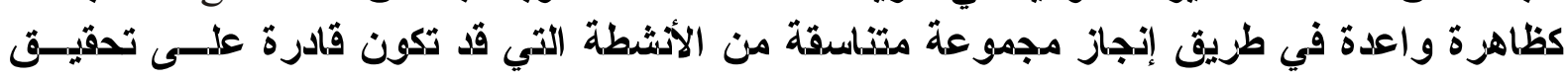
اهتمام مزكز على نطاق أوسع.

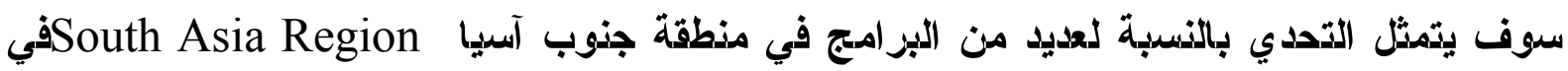

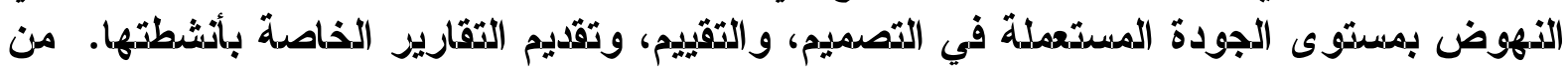

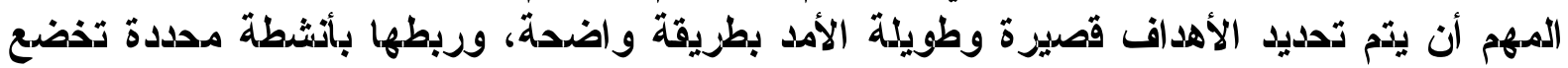

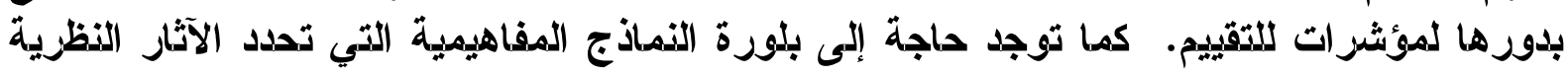

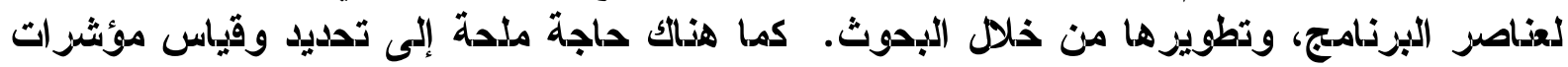

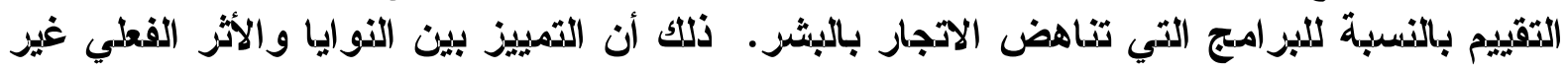

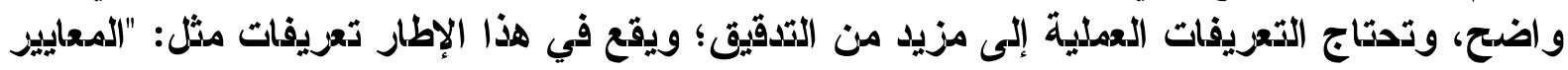

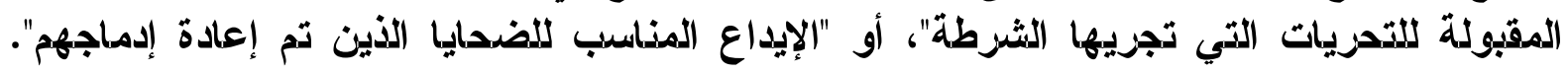

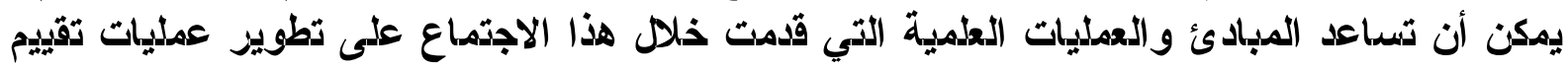

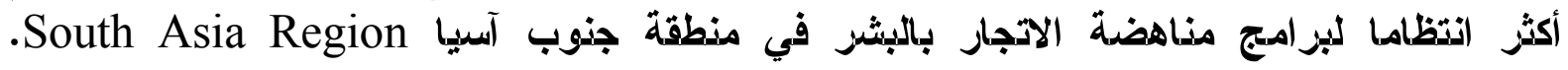

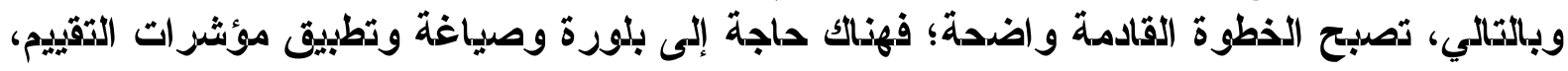




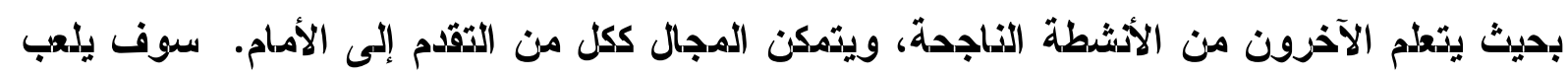

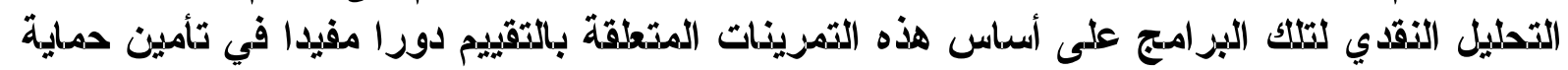

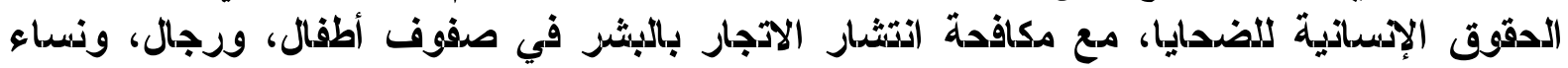
South Asia آسيا جنوب 
For copies of papers please direct responses tothe principal author (refer to list of participants for complete contact information).

Awan, Zia Ahmed

Human Trafficking Has Become a Real

Problem in South Asian Countries including Pakistan.

Chew, Lin

Addressing Trafficking in Persons in the Human Rights Framework.

De Silva, Harenda

Illegal Labour and Commercial Exploitation of Children: Program Models in Sri Lanka

Friedman, S. Matthew

Human Trafficking: Some Inconsistencies with the Sector's Present Definitions and Paradigm

\section{Gallagher, Anne Theresa}

The International Legal Response to Human Trafficking

\section{Ghimire, Durga}

Prevention, Care, Rehabilitation, and Reintegration of Rescued Girls ( $A B C$ 's Experience)
Gramegna, Marco Antonio and Ruben Korevaar

Trafficking Prevention, Protection and Assistance to Victims (presented by Lance Bonneau)

Gupta, Rita Sen

Prevention, Care and Support, and Reintegration: Program Models in Bangladesh

Kapur, Ratna

Tricks and the Law: Legal Regulation of Trafficking, Sex Work and Migration (presented by Roshni Basu)

\section{Malla, Sapna Prathan}

Prosecution on Trafficking Cases: De jure and De facto Reality

\section{O Briain, Muireann}

Trafficked Minors: The Special Case of Children

Rahman, Mizanur

Trafficking in Children: A Shattered Future Bangladeshi Children Working as Illegal Labour or Sex Slaves

Sanghera, Jyoti

Towards the Construction of an Empowered Subject: A Human Rights Analysis of Anti-Trafficking Legal Interventions and Trends in South Asia 
Sinha, Indrani and Sen Roop

Programme Models in India Combating

Trafficking in Children for Commercial

Sexual Exploitation and Illegal Labour 


\section{PARTICI PANTS المشاركون}

\section{Zia Ahmed Awan}

Lawyers for Human Rights and Legal Aide

D-1, 1st floor

Court View Apartment

Karachi, Pakistan

Tel: (92-21) 5685284

Fax: (92-21) 5685938

E-mail:1hrla@fascom.com

\section{Simon Baker}

PATH

Population Council/Horizons

P.O. Box 138 Pratunam

Bangkok 10409, Thailand

Tel: (66-2) 6538586

Fax:(66-2) 2555513

E-mail: simon@popcouncil.th.com

Website: http//www.popcouncil.org

\section{Nandita Baruah}

Regional Project Coordinator

South Asia Anti-Trafficking Project

UNIFEM, 228 Jor Bagh

New Delhi 110003

Tel: (91-11) 469 8297, 4604351

Fax: (91-11) 4622136

E-mail: nandita.baruah@undp.org

Website: http://www.undp.org/unifem

\section{Roshni Basu}

Research Associate

Centre for Feminist Legal Research

Flat no.5, 45 Friend's Colony (East)

New Delhi 110065

Tel: (91-11) 6320499

Fax: (91-11) 6848104
E-mail: cflr45@hotmail.com

Website: http://www.cflr.net

\section{Pankaja Bhattarai}

Program Officer

The Asia Foundation

B.P.O. Box 935

Cha-1 659, Baluwatar

Kathmandu, Nepal

Tel: (977-1) 418-345, 411-458

Fax: (977-1) 415-881

E-mail: nick@taf.org.np

Website: http//www.asiafoundation.org

Katherine Blakeslee

Director

Office of Women in Development

Room 3.08-51

1300 Pennsylvania Avenue, NW

Washington, D.C. 20523-8100

U.S.A.

Tel:(Off)(202) 7120570

Tel:(Res)(202) 2652193

Fax: (202) 7123173

E-mail: kblakeslee@usaid.gov

\section{Lance Bonneau}

Senior Programme Development Officer International Organization of Migration (IOM)

8th, Floor, Kasemkij Bldg.

120, Silom Road

Bangkok 10500

Thailand

Tel: (662) $2353538 / 39$ x 13

Fax: (662) 2367128

E-mail: lbonneau@iom.int

Website: http://www.iom.net 


\section{Lin Chew}

Asian Human Rights Commission

Unit D, 7/F Mongkok Commercial Center

16-16B, Argyles Street

Kowloon, Hong Kong SAR

Tel:(Off)(852) 2698-6339

Tel:(Res)(852) 2982-4428

Fax: (852) 2698-6367

E-mail: clchew@ahrchk.org

\section{Suzanne Cluett}

Associate Director

Bill \& Melinda Gates Foundation

P.O. Box 23350

Seattle, WA 98102

U.S.A.

Tel: (206) 7093100

Fax: (206) 7093185

E-mail: suzanne@gatesfoundation.org

Website: http://www.gatesfoundation.org

\section{Shanthi Dairam}

Director

IWRAW - ASIA PACIFIC

2nd Floor, Block F Anjung FELDA

Jalan Maktab

54000 Kuala Lumpur

Malaysia

Tel: (Off)(603) 26913292

Tel: (Res)(603) 79587451

Fax: (603) 26984203

E-mail: iwraw@po.jaring.my

Matthew S. Friedman

\section{Celine Costello Daly}

Program Associate

Population Council
53 Lodi Estate

New Delhi 110003

India

Tel: (91-11) 4610913

Fax: (91-11) 4610912

E-mail: cdaly@pcindia.org

Harendra de Silva

Chairman/Professor of Paediatrics

National Child Protection Authority

University of Kelaniya, 205-1, Castle

Street

Colombo 8, Sri Lanka

Tel: (Off)(94-1) 672 911-13

Tel: (Res)(94-1) 675 871, 672193

Fax: (94-1) 672915

E-mail:ncpa@diamond.lanka.net

E-mail:harends@lanka.ccom.lk

\section{Johanna Eriksson}

Assistant Program Officer

Child Protection Unit

UNICEF ROSA

P.O. Box 5815, Lekhnath Marg

Kathmandu, Nepal

Tel:(Off)(977-1) 419471 / 419082

Fax: (977-1) 419479

E-mail: jeriksson@unicef.org

\section{Matthew S. Friedman}

USAID

United States Embassy

GPO. Box 2593, Baridhara

Dhaka -1000, Bangladesh

Tel: (880 2) 88247009

Fax: (880 2) 8823648

E-mail:mfriedman@usaid.gov 
Anne-Therese Gallagher

Advisor on Trafficking to the High

Commissioner for Human Rights

Office of the UN High Commissioner for Human Rights

Palais des Nations, 1211 Geneva 10

Geneva, Switzerland

Tel: (41 22) 9179276

Fax: (41-22) 9179018

E-mail: agallagher.hchr@unog.ch

\section{Durga Ghimire}

Director, ABC-Nepal

G.P.O. Box. 5135

Kathmandu, Nepal

Tel:(Off)(977-1) 630 346;

Tel:(Res)(977-1) 330222

Fax: (977-1) 630072

E-mail: abc@transit.wlink.com.np

\section{Philip Guest}

Program Associate

Population Council

P.O. Box. 138 Pratunam

Bangkok - 10409, Thailand

Tel: (Off) (66-2) 2514766 / 2517066

Tel: (Res) (66 2) 6447812

Fax: (66-2) 2555513

E-mail: philip@popcouncil.th.com

Website:

http://www.un.or.th/TraffickingProject/

\section{S. K. Guha}

Senior Program Officer

UNIFEM, South Asia Regional Office

228, Jor Bagh

New Delhi 110003 India

Tel: (91-11) 469 8297, 4604351
Fax: (91-11) 4622136

E-mail: s.k.guha@undp.org

Website: http://www.undp.org/unifem

\section{Sabin Gurung}

Program Officer

Maiti Nepal

P.O. Box 9599

Kathmandu, Nepal

Tel: (977-1) 475316

Fax: (977-1) 492055

E-mail: maiti@ccsl.com.np

\section{Dale Huntington}

Senior Associate

FRONTIERS Associate Director

Population Council

53 Lodi Estate

New Delhi 110 003, India

Tel: (Off)(91-11) 4610913

Tel: (Res)(91-11) 4673930

Fax: (91-11) 4610912

E-mail: dhuntington@pcindia.org

Website: http://www.popcouncil.org

\section{Renu Jain}

Division Chief OSD

USAID

U.S. Embassy

Chanakyapuri

New Delhi, India

Tel:(Off) (91-11) 4198550

Tel:(Res) (91-11) 3275236

Fax: (91-11) 4198454

E-mail: rejain@usaid.gov 
Amita Joseph

Regional Program Coordinator

USAID, American Embassy

Chanakyapuri

New Delhi, India

Tel: (Off) (91 11) 4198706

Tel: (Res) (91-11)686 7534

Fax: (91 11) 4198454 / 8612

E-mail: ajoseph@usaid.gov

Naheed Khilji

Vision

140-B, Model Town

Lahore, Pakistan

Tel: (Off) (92-42) 5885170

Tel: (Res)(92-42) 585374

E-mail: vision@nexlinx.net.pk

\section{Keith Leslie}

Country Director

Save the Children Federation U.S.A.

Maharajganj

P.O. Box 2218

Kathmandu,

Nepal

Tel: (977-1) 412447 / 412598

E mail: kleslie@savechildren.org.np

Website: http://www.savethechildren.org

\section{Vaishali Sharma Mahendra}

Program Officer

Population Council, Horizons

53 Lodi Estate

New Delhi, India

Tel: (91-11) 4610913

Fax: (91-11) 4610912

E-mail: vmahendra@pcindia.org

Website: http://www.popcouncil.org

\section{Sapana Pradhan Malla}

Co-ordinator

Forum for Women Law and Development

KA-1-105, Thapatali

P.O. Box 2923

Kathmandu, Nepal

Tel: (977-1) 242683 / 242 904/ 266415

Fax: (977-1) 240627

E-mail: fwld@fwld.wlink.com.np

\section{Phil Marshall}

Manager

UN Inter-Agency Project on Trafficking of Women \& Children in Mekong SubRegion

UN Building 14th floor, Rajdamnern Nok Ave.,

Bangkok 10200, Thailand

Tel: (Off)(66-2) 2882310

Tel: (Res)(66-2) 8633492

Fax: (662) 2800268

E-mail: phil.marshall@un.or.th

Website:

http://www.un.or.th/TraffickingProject/

\section{Alice Marie Miller}

The Joseph I. Mailman

School of Public Health

Columbia University

60 Haven Avenue, B-2

New York, NY 10032

Tel: (212) 3045280

Fax: (212) 3057024

E-mail: am808@columbia.edu 


\section{Muireann O Briain}

Executive Director

ECPAT International

328 Phayathai Road

Bangkok 10400, Thailand

Tel: (Off)(662) 2153388 ext 104

Tel: (Res)(662)222 5123 ext 419

Fax: (662) 2158272

E-mail: ecpatbkk@ksc15.th.com

Tashia Ann Petersen

Program Officer

IWRAW ASIA PACIFIC

2nd Floor, Block-F Anjung FELDA

Jalan Maktab

54000 Kuala Lumpur

Malaysia

Tel: (Off)(603) 26913292

Tel: (Res)(603) 79587451

Fax: (603) 26984203

E-mail: iwraw@po.jaring.my

Gerald Jacob Pinto

Program Officer - Child Protection

UNICEF

73 Lodi Estate

New Delhi 110003

India

Tel: (91 11) $4690401 / 1401$ ext. 1365

Fax: (91 11) 4627521

E-mail: gpinto@unicef.org

Website: http://www.unicef.org

Jacqueline Pollock

MAP (Migrant Action Program)

P.O. Box 7

Chiang Mai University
Chiang Mai 50200

Thailand

Tel:(Off) (66-53) 811202

Tel:(Res) (66-53) 278422

Fax: (66-53) 811202

E-mail: jackie_pollock@yahoo.com

E-mail:mapnet@cm.ksc.co.th

\section{Mizanur Rahman}

Project Director, NCPTWC Project

ATSEC Bangladesh Chapter

House No. 30 Road 9A, Dhanmondi R/A

Dhaka 1209

Bangladesh

Tel:(Off) (880-2) 8121176 / 9134155

Tel:(Res) (880-2) 8614596

Fax:(880-2) $8121176 / 9134155$

E-mail:mitrec@bangla.net

E-mail: atsecbd@bdmail.net

\section{Bimal Rawal}

National Program Coordinator Nepal

Chapter

Sub-Regional South-Asian Trafficking

Program

International Labor Organization (ILO)

P.O. Box 8971

Kathmandu, Nepal

Tel: (977-1) 528 514, 533933

Fax: (977-1) 531332

E-mail: bimal@ilo.org

\section{Jyoti Sanghera}

Associate

Centre for Feminist Legal Research

Flat No. 5, 45 Friends Colony (East)

New Delhi 110065

Tel: (91 11) 6320499 / 6327303 
Fax: (91 11) 6848104

E-mail: sanghera@id.eth.net

E-mail:jyogaatw@excite.com

Website: http://www.cflr.net

\section{Sankar Sen}

Institute of Social Sciences

8 Nelson Mandela Road

New Delhi 110070

India

Tel: (91-11) 612 1909/ 6121902

Tel: (Res): 6968198

Fax:(91-11) 6137027

E-mail: iss@nda.vsnl.net.in

E-mail: sankars@ndf.vsnl.in

\section{Rina Sen Gupta}

International Organization of Migration

Road No. 50 House No. 3 A

Block NW (G) Gulshan 2

Dhaka

Bangladesh

Tel:(Off) (880-2) 881-7699, 8814604

Tel:(Res) (880-2) 8114627

Fax: (880-2) 881-7701

E-mail: rina@bol-online.com

E-mail: iomdhaka@bil-online.com

Website: http://www-iom.int

\section{Madhuri Rana Singh}

Anti-Trafficking Program Manager

Office of Health and Planning

USAID Nepal

P.O. Box 5653, Rabi Bhawan

Kalimati, Kathmandu

Nepal

Tel: (977-1) 270144

Fax: (977 -1) 272357
E-mail: msingh@usaid.gov

\section{Indrani Sinha}

Executive Director

ECPAT

6 / 4 Lake East 4th Road

Ground Floor Block III

Santoshpur

Calcutta 700075, India

Tel: (91 33) 4165919 / 4633

Fax: (91 33) 4167294 / 4735619

E-mail: sanlaap@giasc101.vsnl.net.in

\section{Catherine Thompson}

Technical Advisor

Office of Health and Planning

USAID Nepal

P.O. Box 5653, Rabi Bhawan

Kalimati, Kathmandu

Nepal

Tel: (977-1) 270144

Fax: (977-1) 527266

E-mail: cathompson@usaid.gov

\section{Archana Tamang}

Regional Alliance Program Coordinator International Save the Children Alliance South \& Central Asia Region

Kupondole, Lalitpur

G.P.O. Box 5850

Kathmandu, Nepal

Tel: (Off)(977-1) 527152 / 523924

Tel: (Res)(977-1) 543548

Fax: (977) 527266

E-mail: archana@scfoscar.org.np 
Sangeeta Thapa

Program Officer

UNIFEM

$1 / 2$, Thapathali

Kathmandu, Nepal

Tel: (977-1) 255110

E-mail:

sangeetathapa@unifem.wlink.com.np

Catherine Thompson

S. Thurairaja

Senior State Counsel

Attorney General's Dept.

Colombo 12, Sri Lanka

Tel: (94-1)320 983 (Off)

Mobile: 072404025

Fax: (94-1) 436421 (Off)

E-mail: thurai61@yahoo.com

E-mail: thurairaja@hotmail.com

Technical Advisor

Office of Health and Planning

USAID Nepal

P.O. Box 5653, Rabi Bhawan

Kalimati, Kathmandu

Nepal

Tel: (977-1) 270144

Fax: (977-1) 527266

E-mail: cathompson@usaid.gov 


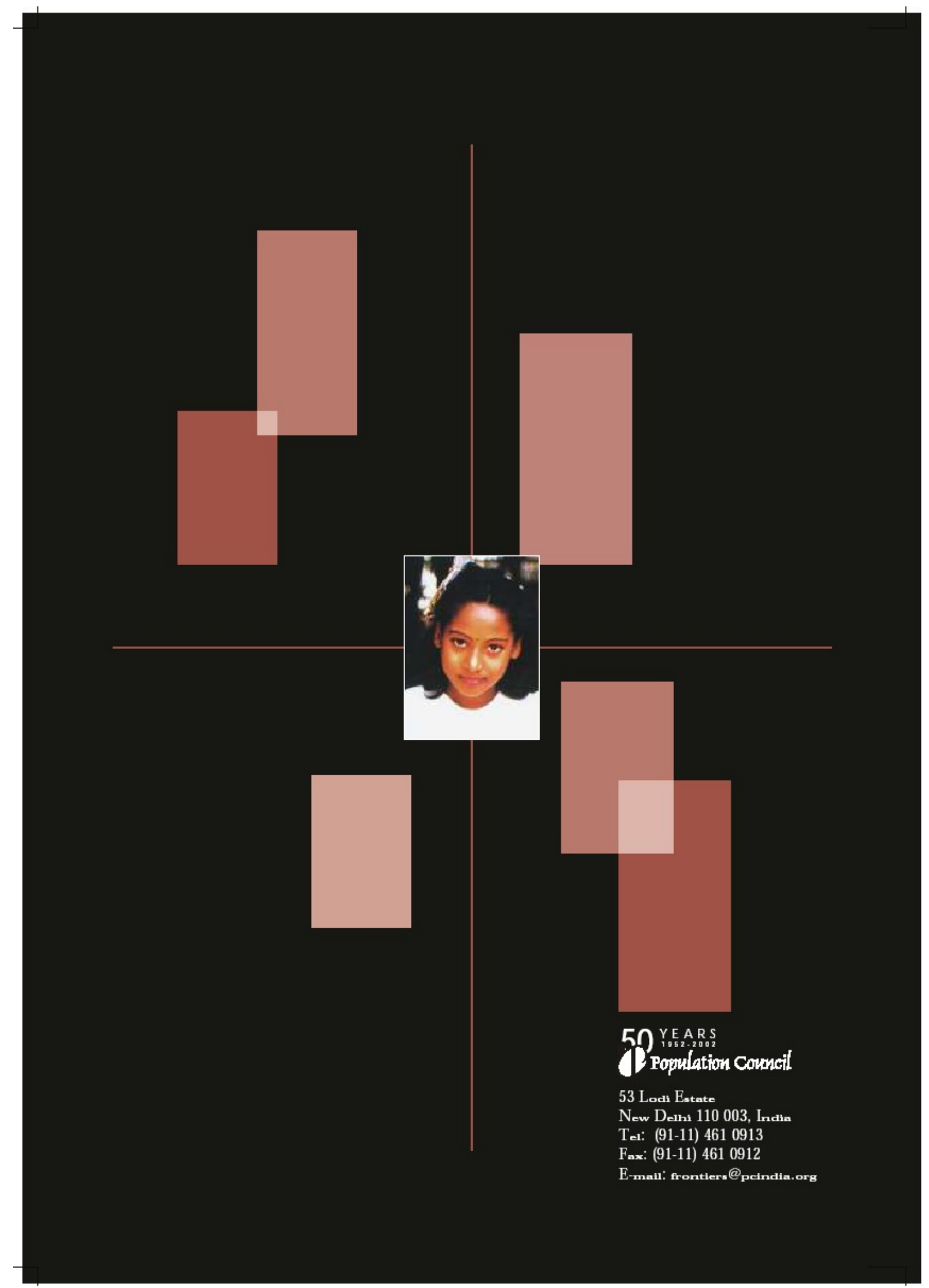

